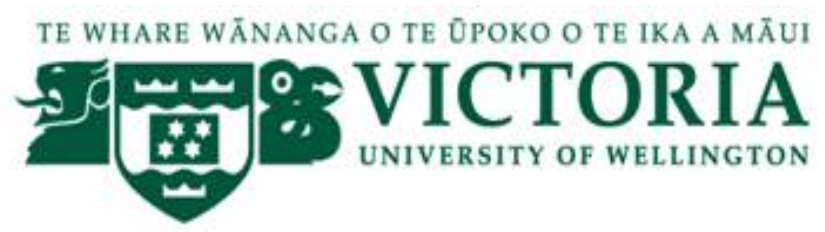

\title{
Transformational Leadership and Organisational Effectiveness in Wellington Secondary School Sport Coordinators
}

Thomas C. Adamson

School of Management

Victoria University of Wellington

PO BOX 56,

Wellington

New Zealand 
Transformational Leadership and Organisational Effectiveness in Wellington Secondary School Sport Coordinators

Thomas Adamson

A dissertation submitted in partial fulfilment of the requirements for the degree of Masters of Commerce in Management at Victoria University, Wellington,

New Zealand 


\begin{abstract}
Leadership is one of the most commonly observed yet least understood constructs in management. Despite this, many will contend that an effective leader will precede an effective organisation. Of the various theories on leadership, the transformationaltransactional model has been promoted as a most desirable and effective style (Bass \& Riggio, 2006; Northouse, 2012). This leadership style has been shown to positively enhance followers' job satisfaction, extra effort and perceived leader effectiveness. Within the New Zealand sporting landscape, and more specifically secondary school sport, a sports coordinator who exhibits transformational leadership may have the potential to overcome a scarcity of resources and empower coaches to perform beyond the resources available and beyond their expectations.
\end{abstract}

This research followed a mixed-methods research design by collecting data through self-administered surveys and semi-structured interviews. At the first stage, a convenience sample of twenty-six Wellington sport coordinators completed a self-rater version of the Multifactoral Leadership Questionnaire (MLQ), in order to determinine their leadership profile, their predominant leadership style and related perceptions of organisational effectiveness. As a second stage, semi-structured interviews with four sport coordinators provided a richer and deeper understanding of the school sport environment and how leadership can positively affect outcomes.

More than a decade earlier, Pope (2002) claimed that secondary school sport had seldom been the subject of attention for researchers, particularly within New Zealand educators. To this day, there is still a limited knowledge base; therefore, the aim of this research was therefore to determine what constitutes an effective leader in the New Zealand Secondary School Sport context. In addition, findings are intended to provide insight about which leadership attributes are valued as contributing to a sports coordinators' ability to manage their school's sporting provision effectively.

Findings show that the transformational leadership behaviours of secondary school sports coordinators are significantly related in several ways to aspects of organisational effectiveness. By contrast, the research also finds that coordinators who exhibited management-by-exception and laissez faire leadership styles and behaviours were perceived as less effective, whilst also having minimal positive impact on coach job satisfaction and exertion of extra effort by followers and others in the system. Of particular interest are findings that indicated transformational leaders are perceived as most effective, especially in 
attracting and retaining coaches, whilst specific behaviours such as contingent reward behaviours may also impact coaches to exert extra effort in executing their roles.

Practical implications are drawn from the findings and are provided to assist secondary schools in considering how to ensure quality and sustainability in sporting provision, and how to seek, recruit, promote, and retain sports coordinators who exhibit transformational leadership behaviours. The insights provided may also allow researchers and educators to better understand the relevance of leadership styles in influencing different aspects of organisational effectiveness in secondary school sport.

The research was necessarily limited to secondary school sport in the Wellington region of New Zealand, and generalisability is only possible to the extent that the research complements other studies. The research was also limited by time, and access to relevant secondary school sport coordinators. Nevertheless, the research was intended to explore a previously under-researched domain, and succeeds in this matter. However, future research would benefit from increasing sample size, greater cross-sectional representation of schools, and employing ethnographic research methodology to assist in explaining the phenomenon further and to a greater depth. Additionally, it is recommended to utilise alternative and more comprehensive conceptions of organisational effectiveness to more fully understand the consequences of leadership behaviours. 


\section{ACKNOWLEDGEMENTS}

The academic journey I have so far embarked on, culminating in this thesis, would not have been possible without the guidance and support from a number of people. I feel they have helped me grow and mature in so many ways. I only wish I could acknowledge everyone that has contributed to this project, as even their slightest actions have left lasting impressions on me.

Thank you to the School of Management at Victoria University for providing with this amazing opportunity to complete my Masters of Commerce. The quality of lecturers and fellow staff members truly helped me settle in to the new environment.

To Professor John Davies, it has been a privilege to work with you over the past year. Your work ethic has been something I will strive to emulate. Thank you for your constant guidance and support, I hope this is not the last piece of work we do together.

Last but not least, I would like to thank my family for their guidance, support and love over the last four years. Mum, Dad, Jimmy, and Jess, you have always been there for me and encouraged me to strive for excellence and reach my potential; I will forever be grateful for that. 
ABSTRACT

ACKNOWLEDGEMENTS iii

TABLE OF CONTENTS .iv

LIST OF TABLES ..vii

LIST OF FIGURES viii

Chapter I: INTRODUCTION. 1

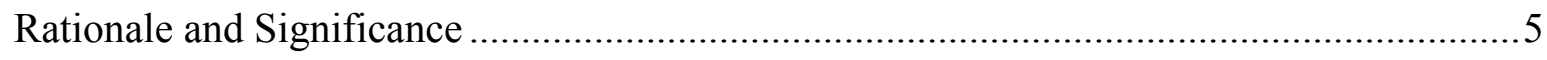

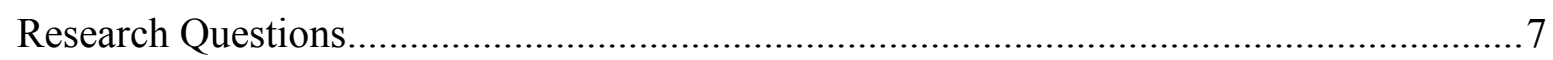

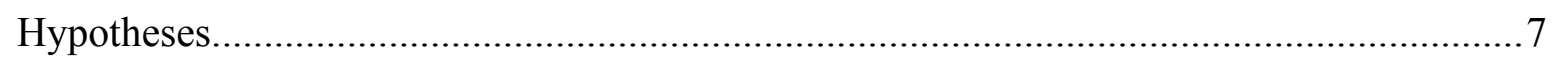

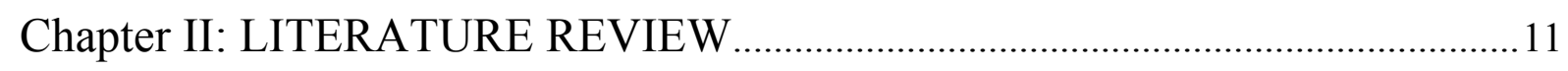

Brief History of Leadership Theories ............................................................................. 11

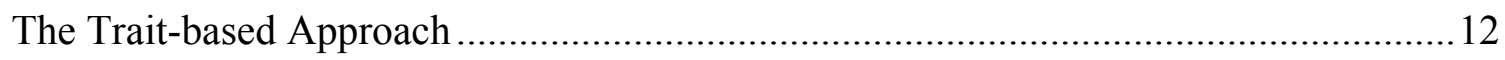

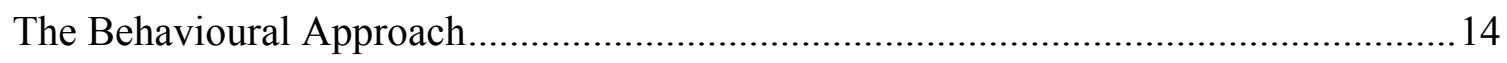

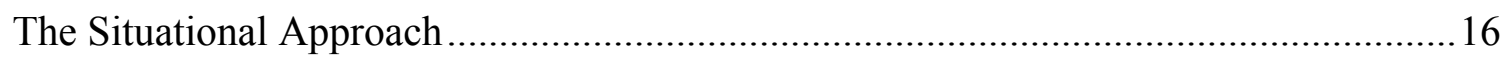

Full Range of Leadership/ New Leadership Theory ..................................................... 17

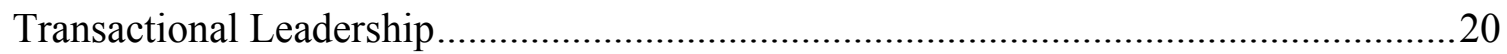

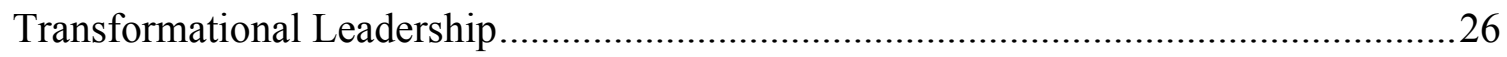

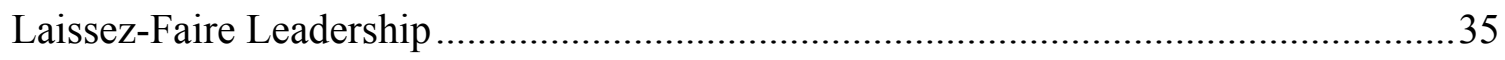

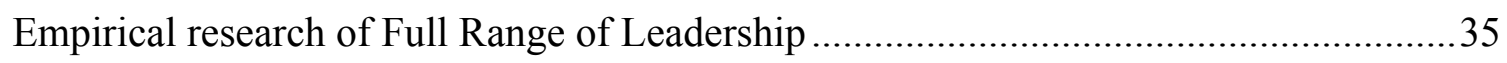

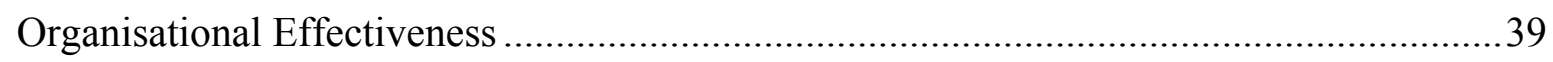

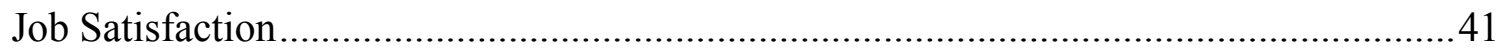




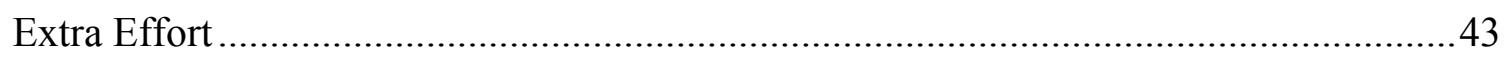

Perceived Leader Effectiveness..................................................................................... 44

Leadership in Sport, Schools and New Zealand................................................................48

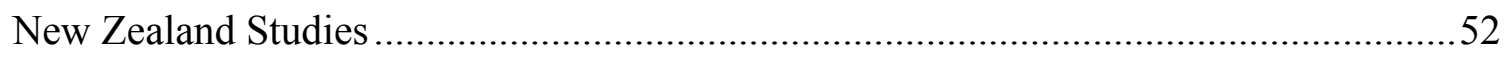

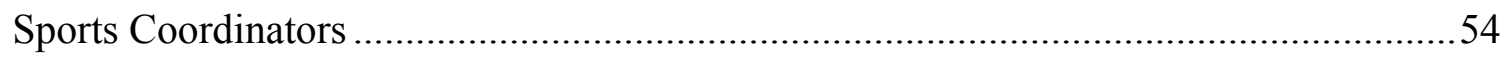

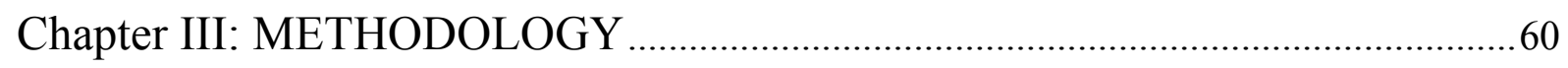

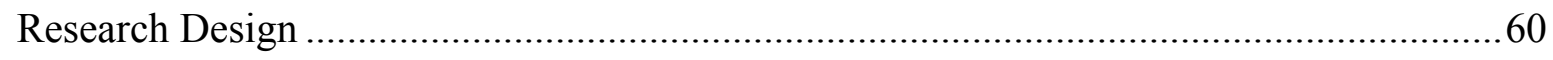

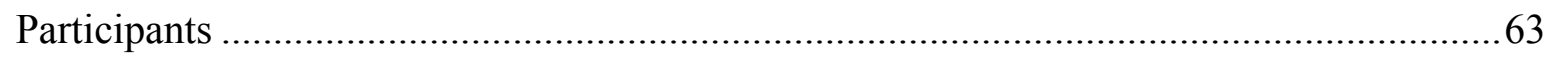

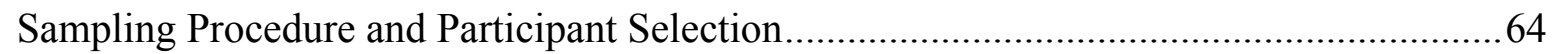

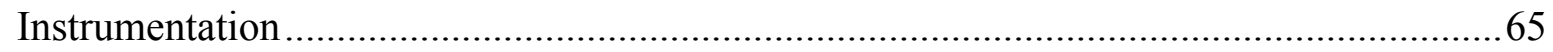

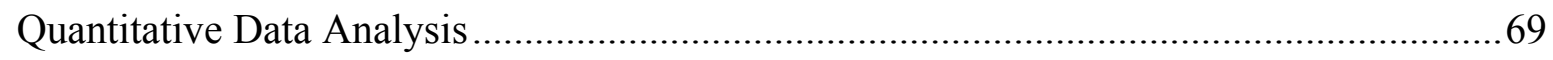

Qualitative Data Analysis .........................................................................................

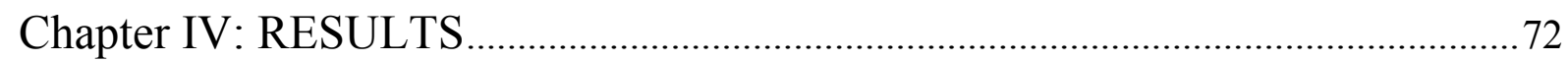

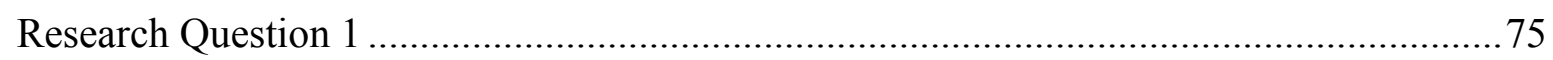

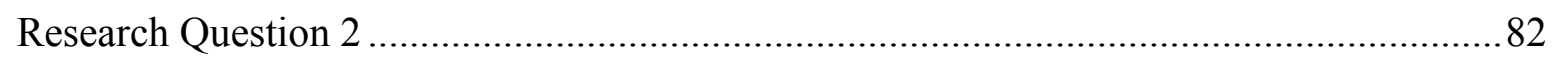

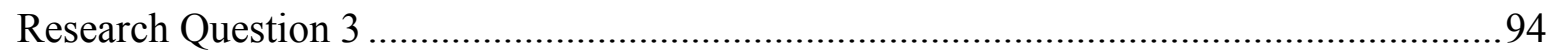

Chapter V: DISCUSSION AND CONCLUSION ……………………………......102

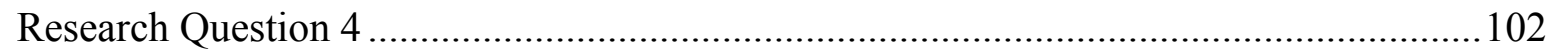

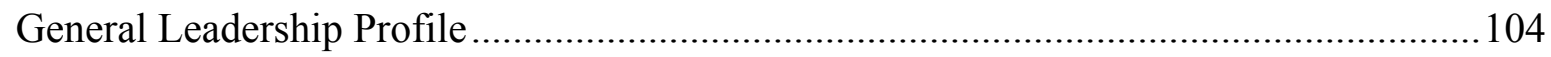

Leadership subscale frequencies and means ................................................................106

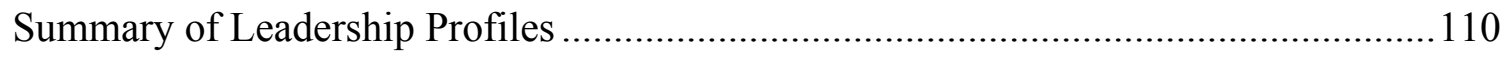

Relationships between leadership behaviours and organisational effectiveness.................111

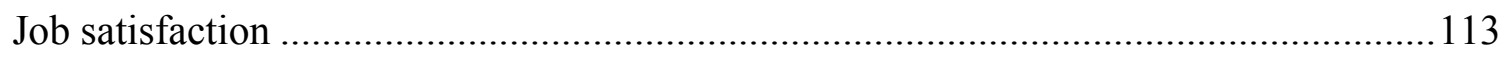

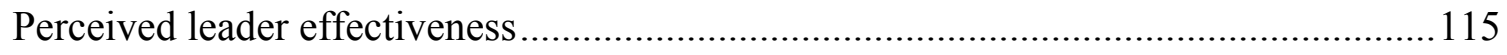

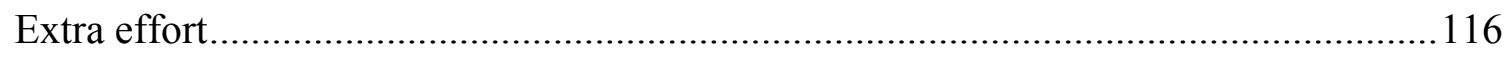




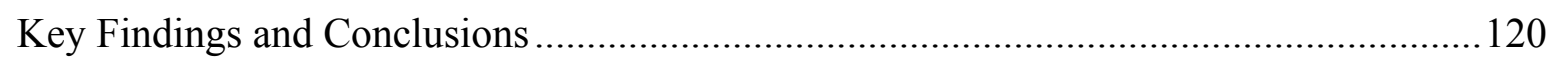

Significance and relevance of leadership in Wellington secondary school sport............121

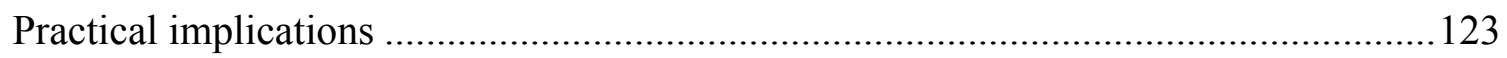

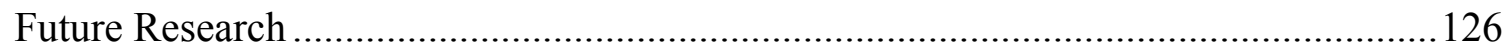

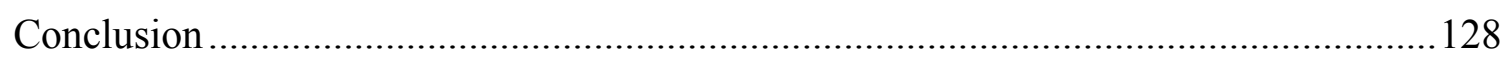

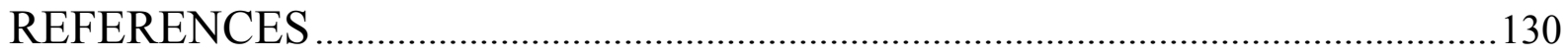

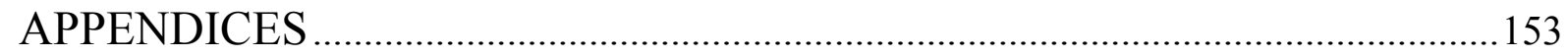

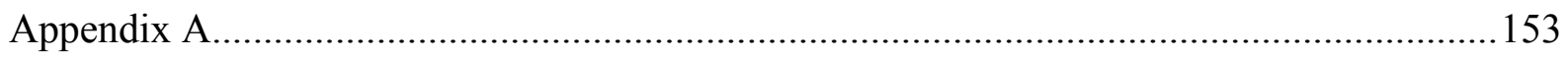

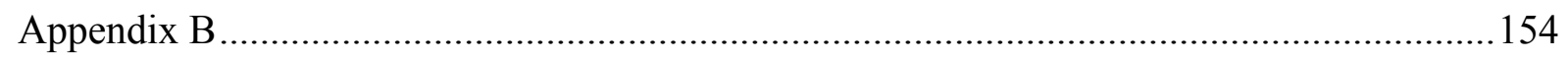

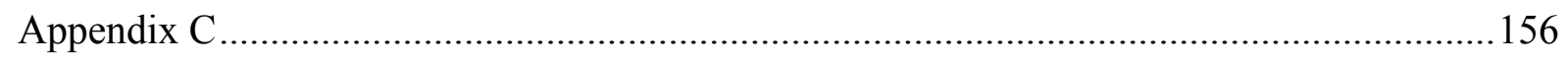

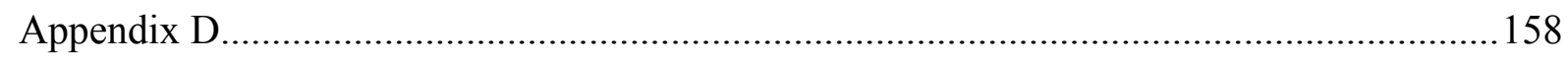

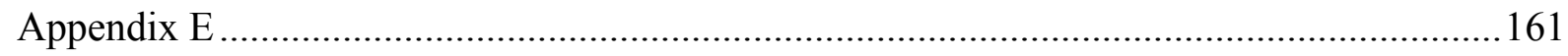




\section{LIST OF TABLES}

TABLE 1. Scoring of MLQ 5X-Short Self-Rater Instrument.

TABLE 2. Cronbach's Alpha scores for MLQ sub-scales. 73

TABLE 3. Wellington Secondary School Sports Coordinator Responses to the MLQ leadership items. 76

TABLE 4. Wellington Secondary School Sports Coordinator Leadership Sub-scales .77

TABLE 5. Organisational Effectiveness Measures as rated by Sports Coordinators. 83

TABLE 6. Matrix correlation between sport coordinators' self-rated leadership styles and organisational outcomes. 


\section{LIST OF FIGURES}

FIGURE 1. Frequency of mean leadership behaviours exhibited by Wellington Secondary

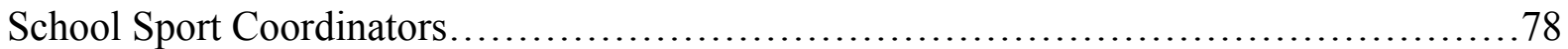




\section{Chapter I: INTRODUCTION}

This study explores an important topic in sport, in education and in the management and leadership of sporting organisations in an educational context. In particular, it examines the specific leadership behaviours of sport coordinators within a secondary school sport context and how these behaviours affect organisational effectiveness of their school's sporting department.

In subsequent sections, we outline the context of the study, the author's rationale for the study, and the research questions and hypotheses this study seeks to answer. We conclude the chapter with a summary, and with a further outline of thesis structure.

Leadership has long been considered one of the most commonly observed yet least understood constructs in business management (Burns, 1978). Indeed, and perhaps as a consequence numerous conceptual developments and operational definitions have been advanced over time relating to the notion of leadership (Milne, 2007), evolving from considerations of trait, to behavioural aspects, to situational features, and now to transformational/transactional dimensions of leadership. The purpose of these conceptualisation, conceptual frameworks or theories has been to determine whether and when managers possess and display leadership qualities, as a seeming complement to aspects of the managerial role which would require more than simply supervising and directing staff. Indeed, Bennis and Nanus believed it to be important to differentiate between managing and leading, and managers and leaders, stating that, "managers are people who do things right and leaders are people who do the right things" (1985, p. 21). In articulating a simplified point of 
difference, it has been stated that managers may perceive workers more as a means to an end, whereas leaders may view people as an end in themselves.

The efficacy of contemporary organisations may depend on its leaders' abilities to formulate a vision, and implement it Bennis $(1959 ; 1984 ; 2001)$. Vision is defined as "the capacity to create and communicate a compelling picture of a desired state of affairs, impart clarity to this vision, and induce commitment to it" (Bennis \& Nanus, 1985, p. 181). Due to modernisation of industries, many leaders are required to modify their existing leadership styles to accommodate increasingly competitive environments and changing needs of followers (Yukl, 1989). However, the traditional leadership styles of business management have been deemed by scholars (Burns, 1978; Bass, 1985) to be inadequate for reacting to environmental changes, let alone shaping and creating a desirable vision for their future.

It is claimed, for example, the capacity of a leader to influence subordinates to act towards a common goal is vital for the effectiveness of any and all organisations. This is especially so, when sport organisations are undergoing rapid change and are operating in a turbulent environment (Soucie, 1994; Weese, 1996). The pace of change that confronts sport managers requires adaptive and effective leadership (Bass, Avolio, Jung, \& Berson, 2003). Sport organisations will therefore need to actively seek to identify, attract and retain leaders who possess these qualities as they seek to ensure survival and quality of provision (Soucie, 1994).

Sport management has seen a shift in focus to become more business-like and incorporate private sector practices in the way they manage their employees (Chelladurai, 2001). For example, in response to the changing environment in which they operate, sport managers are seeking to adopt and promote new leadership styles to ensure their organisations remain effective. However, several conceptualisations of leadership relating to traits (Stogdill, 1948), behavioural aspects (Yukl, 2001), and situational contexts (Yukl, 1989) were 
considered to be theoretically flawed and narrow in understanding or guiding approaches to leadership. Subsequently, Bryman (1992) outlined the need for a new theoretical framework capable of examining the traits and behaviours of leaders in a range of situations.

Interpreting the shortcomings of the aforementioned approaches of studying leadership, Burns (1978) contended that leadership in modern organisations couldn't be properly understood when employing these singular frameworks or models. At the time, he believed the transactional nature of leadership that had previously underpinned managerial thinking was no longer appropriate as a response to globalisation and cultural changes with businesses in the 1970s. Thus, Burns (1978) conceived an integrative theory of leadership that constitutes a bi-directional leader-follower relationship that recognises interdependency and the need for reciprocal action in leader-followers when attempting to achieve a common goal. In effect, Bass (1985) contributed to the operationalization of Burns' initial work and presented a formal theoretical framework that he termed transformational leadership.

Transformational leadership, since, emerged as what is regarded as a desirable strand of leadership that seeks to improve followers' job satisfaction, to promote extra effort whilst embracing organisational goals and vision, and in turn seeking to enhance commitment and loyalty to the organisation (Bass \& Rigio, 2006). Thirty years of research examining the transformational theory or model of leadership has elevated transformational leadership to being a most desirable (Den Hartog et al., 1999; Leong \& Fischer, 2010; Singer, 1985) and effective (Bass \& Riggio, 2006) style appropriate for use in a range of organisational settings (Bass, 1997; Yukl, 1989). Meta-analyses of the literature by authorities in the field (Bass, 1999; DeGroot et al., 2000; Lowe et al., 1996; Judge \& Piccolo, 2004; Wang, Oh, Courtright, \& Colbert, 2011) have also shown that transformational and transactional leadership may positively predict a range of organisational outcomes - including followers' job satisfaction, 
extra effort and perceived leader effectiveness - that contribute to greater performance and effectiveness.

Complementing findings from research in private and public setting, the utility of transformational leadership in sport organisations is also apparent. Several scholars have suggested that sport administrators who exhibit the transformational leadership style may be able to increase motivation and commitment of followers in their pursuit of greater quantity and quality of provision (Aminuddin, 1998; Slack \& Parent, 2006; Weese, 1994). Indeed, research has shown that the transformational leadership appears to be the most prevalent and effective style for achieving organisational effectiveness in sport organisations (Armstrong, 1992; Weese, 1995; Doherty, 1997; Doherty \& Danylchuk, 1996; Ristow et al., 1999). These findings subsequently led Gilbertson and colleagues to emphasise the suitability of transformational leadership for New Zealand sporting organisations, "Given the very significant changes in our society and in the roles of sport and recreation, the authors believe that transformational leadership is the most relevant domain of study for leaders of New Zealand sport and recreation" (2000, p. 154).

A recognition that school sport coordinators in New Zealand are required to fill leadership roles, can be determined from work activities and job role descriptions specifying a need to formulate, coordinate, and lead programmes. Additionally, their roles demand an understanding of educational curriculum and sporting needs of youth, along with the ability to motivate and influence volunteers to contribute their time and efforts (New Zealand Secondary School Sports Council, 2011; Sport NZ, 2008, 2014). These broad aims and objectives must often be accomplished in creative ways and constrained circumstances, described as doing more with less as sport coordinators seek to collaborate with both internal and external entities to enhance their limited financial, structural and personal capacities (Marshall \& Hardman, 2000; Pope, 2011). It is not surprising then, that those involved in 
school sport may themselves recognise the need to identify, promote, and retain personnel who express transformational leadership.

The purpose of this study was to identify the strengths and weaknesses of leadership within the Wellington Secondary School Sport Coordinator community, examine the relationship that leadership has on specific measures of organisational effectiveness, and determine the leadership behaviors that are perceived to be most impactful and most valued. The central problem of this study has sought to address whether sports coordinators who lead an effective organisation exhibit characteristics of transformational leadership behaviours. This research problem is examined in a context where secondary school managers or administrators may not be aware of the leadership behaviours of sport coordinators or whether they are successfully using effective leadership styles to attract, retain, and satisfy the needs of coaches.

As this is a previously unexplored context, the study has exploratory and explanatory purposes and seeks not only to situate the Wellington Secondary School context within the contexts of the existing literature, but also to determine commonalities and differences in context and finding. As such, there is an expectation that results from this study may be of use to a range of New Zealand sporting bodies such as Sport NZ or the New Zealand Secondary School Sports Council that have responsibility for and can inform current and influence future leadership practices in school sport.

\section{Rationale and Significance}

The rationale for seeking to make this potential contribution comes from an identified research gap, namely the lack of literature related to leadership in the field of secondary school sports within New Zealand. More than a decade ago, Pope (2002, p. 90) had stated, "secondary school sport has seldom been the subject of systematic research from educators and academics". This was despite research showing that "more than half $(60 \%)$ of students 
participate in competitive secondary school sport; the popularity of organised sport is clearly evident" (New Zealand Secondary Schools Sport Council, 2001). However, one issue in addressing the needs of such participant students is that many schools have long faced not only declining budgets but the declining availability of skilled coaches (Committee on Sports Medicine \& Committee on School Health, 1987; Pope, 2002). More recently, and perhaps not surprisingly, Pope (2011) has reported a serious and broader decline in the status of school sport that he believes is in need of urgent action and improvement of resources.

It is therefore of importance, given the hurdles and barriers presented by such specific and broader decline of support and status, to examine the behaviours and effectiveness of those individuals, within schools, for example, sports coordinators, who shoulder the responsibility and who are expected to provide direction to the organisation and delivery of sport and sporting opportunity. It may not be surprising that the New Zealand Secondary School Sports Council recognise that, in such circumstances, sport coordinators need to be able to run their sporting department with greater direction, purpose, and vision (2011). As these coordinators are expected to, and can play an integral role in quality provision, the question therefore arises of whether effective leadership of a transformational or transactional nature is more likely to overcome these barriers, and create the associated benefits of perhaps inspiring followers such as coaches to more concerted effort, by increasing job satisfaction, and by behaving so as to be perceived as an effective leader. In turn, these behaviours and outcomes will perhaps contribute to greater organisational effectiveness, as a demonstration of how school sport leaders can surmount the barriers they face in attempting to provide quality-sporting provision to youth. 


\section{Research Questions}

Given the need to gather information about leadership behaviours and practices exhibited by New Zealand secondary school sport coordinators, the following key research questions were designed to understand, explain, and evaluate this phenomenon. They are as follows:

1. What are the leadership profiles of secondary school sport coordinators in the Wellington region?

a. To what degree to sports coordinators exhibit transformational leadership behaviours?

2. Are there any significant relationships between sport coordinators perceived leadership styles (transformational, transactional and laissez-faire) and organisational effectiveness measures (coaches' job satisfaction, extra effort and leader effectiveness)?

3. What leadership behaviours do sports coordinators identify as most valuable to the effectiveness of their school sport provision?

4. Confirming or not the applicability of previous studies frameworks and findings on transformational, transactional, and laissez-faire leadership and organisational effectiveness to the context of Wellington Secondary School Sport.

\section{Hypotheses}

The following hypotheses have emerged from the literature review (See Chapter II) and relate to identified gaps in research on secondary school sport, secondary school sport coordinators, and the Wellington secondary school sport context. Whilst they are stated here, the rationale for their use is provided throughout Chapter II.

As such, all hypotheses are concerned with Wellington secondary school sports coordinators and their respective followers. Hypothesis testing is conducted in a standard 
manner using statistical significance at the $p<0.05$ level, that is, the null hypothesis will be rejected if explanations of observed data rely on chance explanations that involve less than 0.05 chance/probability.

\section{Transformational Leadership and Organisational Outcomes}

$H_{0}$ 1: There is no relationship between sports coordinators who exhibit transformational leadership and coaches' extra effort.

$H_{o} 2$ : There is no relationship between sports coordinators who exhibit transformational leadership and coaches' job satisfaction.

$H_{o} 3$ : There is no significant relationship between sports coordinators who exhibit transformational leadership and perceived leader effectiveness.

\section{Transformational leadership Sub-Scale Behaviours}

$H_{o} 4$ : There is no significant relationship between sports coordinators who exhibit idealised influence (attributed) behaviours and the organisational outcomes

$H_{o}$ 5: There is no significant relationship between sports coordinators who exhibit idealised influence (behaviours) behaviours and the organisational outcomes

$H_{o} 6$ : There is no significant relationship between sports coordinators who exhibit inspirational motivation behaviours and the organisational outcomes

$H_{o}$ 7: There is no significant relationship between sports coordinators who exhibit intellectual stimulation behaviours and the organisational outcomes

$H_{o} 8$ : There is no significant relationship between sports coordinators who exhibit individualised consideration behaviours and the organisational outcomes

\section{Transactional Leadership and Organisational Outcomes}

$H_{o}$ 9: There is no relationship between sports coordinators who exhibit transactional leadership and coaches' extra effort.

$H_{0}$ 10: There is no significant relationship between sports coordinators who exhibit transactional leadership and coaches' job satisfaction.

$H_{0}$ 11: There is no significant relationship between sports coordinators who exhibit transactional leadership and perceived leader effectiveness. 


\section{Transactional Leadership Sub-Scale Behaviours}

$H_{o}$ 12: There is no significant relationship between sports coordinators who exhibit contingent reward behaviours and the organisational outcomes

$H_{o}$ 13: There is no significant relationship between sports coordinators who exhibit active management-by-exception behaviours and the organisational outcomes

$H_{0}$ 14: There is no significant relationship between sports coordinators who exhibit passive management-by-exception behaviours and the organisational outcomes

\section{Laissez-Faire Leadership and Organisational Outcomes}

$H_{0}$ 15: There is no significant relationship between sports coordinators who exhibit laissezfaire leadership and coaches' extra effort.

$H_{0}$ 16: There is no significant relationship between sports coordinators who exhibit laissezfaire leadership and coaches' job satisfaction.

$H_{o}$ 17: There is no significant relationship between sports coordinators who exhibit laissezfaire leadership and perceived leader effectiveness.

In summary, leadership has been understood as a vital aspect that may contribute to greater organisational success, performance and overall effectiveness. It has been suggested that a leaders' ability to influence subordinates to seek the accomplishment of an organisation's vision may determine effectiveness (Bennis, 1959; 1984; 2001). However, the changing nature of operations and practices within contemporary sporting organisations (Soucie, 1994) requires sport coordinators to exhibit different leadership approach to the traditional transactional style according to Bass (1985).

Subsequently, Bass (1985) presented a formal and operationalised transformationaltransactional leadership theory that has been suggested as a most desirable and effective style (Bass \& Riggio, 2006; Northouse, 2012), particularly within sporting organisations (Weese, 1994). Moreover, Gilbertson and colleagues (2000) have emphasised the suitability of transformational leadership for New Zealand sport coordinators and sporting organisations, as they attempt to do more with less and meet the broad aims and objectives of their role (Sport NZ, 2008; 2014). 
This chapter has identified the gap in secondary school sport literature and outlined how it's rationale for exploring leadership behaviours of Wellington sport coordinators. The following chapters will provide a thorough literature review on leadership styles and their associations with organisational outcomes, particularly within school, sport and New Zealand settings; a detailed look into the methodological structure this research followed for data collection and analysis; and lastly a discussion and conclusion of the implications this research may have on current and future sport coordinators, whilst also acknowledging limitations of the study and providing recommendations for future research. 


\section{Chapter II: LITERATURE REVIEW}

To help formulate the research questions and lines of inquiry for this study, a comprehensive literature review was conducted. The literature covers topics on leadership, and more specifically transformational, transactional, and laissez-faire constructs of leadership; and then how each construct relates to organisational effectiveness in relation to job satisfaction, extra effort and commitment to the organisation; perceived effectiveness of leaders; non-profit and volunteer management; and finally, the status of secondary school sport in New Zealand.

\section{Brief History of Leadership Theories}

Leadership has been long been a popular area of study for management researchers. Over the years a range of constructs, models and theories have arisen creating a maelstrom/plethora of definitions and in turn utility for study (Bass, 1985; Burns, 1978; Bryman, 1992; Conger \& Kanungo, 1987; Davis \& Luthans, 1979; DeRue, Nahrgang, Wellman \& Humphrey, 2011; Kirkpatrick \& Locke, 1991; Yukl, 1989; Zaccaro, 2007). According to Stogdill, "there are almost as many definitions of leadership as there are persons who have attempted to define the concept" (1974, p. 259).

For instance, many key authors assert that leaders induce subordinates to behave in a desired manner through an exertion of their power, trust and respect (Bass \& Stogdill, 1990; Bennis, 1959, 1975; Northouse, 2012; Stogdill, 1974). More specifically, they suggest that leadership involves the relationships and interactions between organisational members - and thus, involves social influence processes (Yukl, 1989). In this regard, "leadership is a process of interaction between leaders and followers where the leader attempts to influence followers to achieve a common goal" (Voon, Lo, Ngui, \& Ayob, 2011, p. 25). Such literature suggests 
that the ability of a leader to influence subordinates to act towards a common goal is vital for the effectiveness of any and all organisations.

Notwithstanding such work, several trends in leadership theorizing have evolved over the past 60 years according to Northouse's Leadership book (2012). The complex and multifaceted phenomenon has seen researchers over time, attempt to define leadership in terms of individual traits, leader behaviours, situational approaches, and also what has been termed the New Leadership approach (Yuk1, 1989). Indeed, Bryman (1992) has presented a timeline illustrating the development of the above theories. It is worthwhile to outline each of the trait, behavioural, and situational approaches as they all contributed to the eventual development of the Full Range Model of Leadership Theory in the early 1980s (Burns, 1978; Bass, 1985).

Over time, researchers have also attempted to apply leadership theories within a range of organisational contexts - from the private sector and military (Bass, 1985, 1998) to educational settings, which was led by Kenneth Leithwood (1994; Leithwood \& Poplin, 1992; Leithwood \& Jantzi, 2005; Steward, 2006), and also to sporting organisations (Chelladurai, 2001; Crust \& Lawrence, 2006; Soucie, 1994). However, as each new theoretical framework was developed to extend earlier work and address 'flaws' in their conception, in time, many were also proven to be inadequate in defining or explaining leadership. Currently, what has become known as the New Leadership/ Full Range of Leadership/ TransformationalTransactional-Laissez-faire theory is argued to effectively integrate previous theorising into one universally acceptable model (Bass, 1985; Burns, 1978; Yukl, 1989).

\section{The Trait-based Approach}

The personal attributes of the leader are the focus of attention in this approach. It was first posited that effective leaders are born with and possess traits that govern their capability to influence, guide, and manage followers (Galton \& Eysenck, 1869). Conversely, it was 
assumed that non-leaders simply don't possess the desirable traits so cannot acquire the ability to be a successful leader. According to Bennis and Nanus (1995), such traits are 'preconceived' and "no amount of learning or yearning could change your fate" $(1985$, p. 5). That is, personal demographics (e.g., gender, age, education), task competence (e.g., conscientiousness, intelligence, motivation), and interpersonal attributes (e.g., extraversion, communication) are 'fixed' variables that are innate.

Over time, researchers perspectives shifted from innate or heritable traits to encompass all enduring traits, redefining leader traits as "relatively coherent and integrated patterns of personal characteristics, reflecting a range of individual differences, that foster consistent leadership effectiveness across a variety of group and organizational [sic] situations" (Zaccaro, 2007, p. 7). Subsequent studies found that traits relating to demographics, technical and social skills and abilities, and personality traits can predict leader effectiveness (Eagly, Karau, \& Makhijani, 1995; Judge, Bono, Ilies, \& Gerhardt, 2002; Judge, Colbert, \& Ilies, 2004). Effective managerial leadership in this conception was then presumed to include "high self-confidence, emotional stability, energy level, initiative, stress tolerance, and favourable attitude toward authority figures" (Yukl, 1989, p. 271). In addition, Kirkpatrick and Locke (1991) identified a complementary set of six traits that characterise how leaders differ from non-leaders: drive, the desire to lead, honesty/integrity, selfconfidence, cognitive ability, and knowledge of the business.

For some researchers, a major flaw of this conceptualisation was that the sole focus of inquiry was on the leader - it ignored any relationships, interactions, environmental conditions and so forth that may impact leader effectiveness. Indeed, on the basis of some widely recognised reviews (Stogdill, 1948), the trait-based approach was deemed insufficient in its ability to delineate leaders and their subsequent effectiveness. Consequentially many 
researchers turned their attention to defining and explaining leadership through a different construct; for example, the behavioural approach that began in the 1950s.

\section{The Behavioural Approach}

Critiques of the leader trait paradigm led to theorizing that whilst managers may possess a range of these preconditions, they cannot be deemed successful if they don't behave appropriately or manage relationships in role. Yukl (1989) identified the approach as emphasizing what leaders and managers actually do in their roles, and the relationship to effectiveness. According to Davis \& Luthans (1979), successful leaders - through brief daily interactions - are able to gather important information on operations as well as the status of their leader-follower relationships. Kotter (1982) goes on to say that such information enables the leaders to be particularly effective in developing objectives and strategies to implement in both the short- and long-term.

The behavioural approach has encompassed a number of theories and frameworks, which can be classified into four main categories: task-oriented behavioural, relationshiporiented behaviours, change-oriented behaviours, and passive leadership (DeRue, Nahrgang, Wellman, \& Humphrey, 2011). Hundreds of studies have explored their utility in a range of organisational settings, rationalising that a "behavior [sic] approach to leadership will allow the observer to see what the manager encounters on a day-to-day basis" (Yukl \& Mahsud, 2010, p. 81). For a comprehensive review, see Burke and colleagues' (2006) meta-analysis of task and relationship-oriented behaviours reviewed the numerous behaviours and their impacts on organisational outcomes.

Most notably, this approach has been attributed as providing a re-orientation of leadership research activities. Bass (1990) claimed this approach to have broadened the scope of leadership inquiry to now include people-oriented activities. Thus, it has been suggested/claimed the development of charismatic leadership (Conger \& Kanungo, 1987) and 
the transformational-transactional theories (Burns, 1978; Bass, 1985) arose from the newly focused relationship-centred approach that arose from behavioural leadership.

It has also been claimed that such a behavioural-approach to theorizing not only provided greater depth in understanding of leadership in a range of contexts, but to a broader acknowledgement of leadership behaviours. As such, this approach afforded practitioners the opportunity to envisage and develop in-house training programmes that could improve the leadership skills of all managers (Day, 2001). Day's (2001) review of leadership development in context outlined a distinction of how such courses can facilitate leader development or leadership development - the former being aimed at improving human capital through raised self-awareness of individual leaders; the latter seeking to enrich the social capital of the organisation as leadership is based on reciprocal relationships and is a shared process.

The propensity for organisations to offer leadership development courses and programmes has been fostered by the findings of empirical research (Day, 2001; O'Neil, Hopkins, \& Bilimoria, 2015; Zenger, Stanholtz. \& Folkman, 2013) supporting the belief that leadership behaviours can be taught. For instance, courses on transformational leadership have been shown to improve visioning and team building dimensions (Kets de Vries, 2009), and also to decrease negative psychological reactions to followers (Mason, Griffin, \& Parker, 2014). According to Stanbrough (2010), such courses can benefit a range of organisational settings including secondary school sports.

In spite of the added benefits that exploration of the behavioural approach afforded leadership knowledge and research, several limitations became apparent through empirical research applied in different organisational settings (Yukl, 2001). For instance, Bass's (1998) application of the Multifactor Leadership Questionnaire (MLQ) surfaced different leadership tendencies and preferences across military, industrial and educational contexts. Several scholars have believed that, in many instances, the environment can determine the fit of 
leadership styles and affect their relative efficacy (Bryman, Stephens, \& Campo, 1996; Yuk1, 1989). Taylor's doctoral dissertation (2014) re-asserts this belief, stating,

“The leader's style is not cut-and-dry; it depends on the environment. One behaviour may be best in one setting and another in a different setting. This, naturally, makes measurements difficult. And as styles changes in different settings, so must the variables" $(2014$, p. 14).

Other prominent authors had previously taken a critical stance that the behavioural approach can fail to integrate intervening contextual variables that impact the leadershipoutcome relationship (Bennis, 1975; Burns, 1978; Yuk1, 1989). In response to these critiques, a new wave emerged focussing on the context and setting of leadership.

\section{The Situational Approach}

Prior failure to comprehensively delineate successful leadership traits has been linked to recognition that "no traits were universally associated with effective leadership and that situational factors were also influential" (Kirkpatrick \& Locke, 1991, p. 48-49). This led to scholars shifting their attention to the contextual and environmental factors that may determine and moderate the effects of leadership behaviours.

According to Yukl (1989), researchers had conducted investigations of the situationalapproach through an examination of the relationships between leadership behaviours and effectiveness variables. According to this lens, a leader's effectiveness is premised on the compatibility of their leadership style to the specific context. For example, Hersey and Blanchard (1988) had found that effective leaders select an appropriate style in accordance to the situation, and based on the level of readiness of the subordinate. They stated that that it is the leader's responsibility to identify and modify their behaviours.

Podsakoff, MacKenzie, Ahearne and Bommer's later work (1995) reviewed situational leadership literature and tested the theoretical and practical importance of a range 
of moderators for leaders. They found that whilst extant theory could explain the effects from simple factors; however, it could not account for or explain the variety of situational or contextual factors that may moderate leadership outcomes - and which could range across the type of organisation (private vs public), level of management, work unit size, time in position, gender of followers, and so forth (Bass, 1998; Bryman, Stephens, \& Campo, 1996; Lowe, Kroeck, \& Sivasubramaniam, 1996; Piccolo \& Colquit, 2007). Nevertheless, the situational approach has been found to have some utility in explaining differences relating to certain categorical variables e.g., public or private organisations. Unfortunately, it has been accepted that the variety other factors that relate to situational context perversely inhibits the explanatory value of context.

Overall, scholars and leaders alike acknowledged that a singular or uniform approach to the conduct of leadership could not lead to successful outcomes in different contexts or organisational settings. Others have also raised doubts about reliance on situational approaches to understanding leadership and leadership effectiveness. Yukl (1989), for example, identified methodological problems and a theoretical failure to present universal principles for tackling an organisational setting. Subsequently, scholars sought a more comprehensive model that is capable of combining general principles and situational elements.

\section{Full Range of Leadership/ New Leadership Theory}

During the 1970s and 1980s leadership researchers began examining the relationship between the leader and followers more closely (Burns, 1978; Bass, 1985). As such, there was a paradigmatic shift from the trait, behavioural, and situational approaches towards a more integrative theory, capable of examining the traits and behaviours of leaders in a range of situations (Bryman, 1992). 
According to Bass (1997), "The old paradigms of task-oriented or relations-oriented leadership, directive or participative leadership, and autocratic or democratic leadership and related exchange theories of leadership ignored effects on leader-follower relations of the sharing of vision, symbolism, imaging, and sacrifice” (p. 133). Moreover, Burns (1978) and Bass (1985) believed the past approaches were too focused on easily observable relationships and exchanges; and that there needed to be a focus on the effects of outliers, too extraordinary leaders. Such beliefs were shared amongst leadership researchers at the time (Conger \& Kanungo, 1987; Northouse, 2012) leading to a shift of focus towards developing a comprehensive leadership theory for an effective relationship between leader and followers that would be predicated on the role of influential leaders (Kent, 1999).

During this time, management researchers became interested in charismatic leadership, and the transformation and revitalization of organisations (Yukl, 1989). Original work by House (1977) - previously published as A 1976 Theory of Charismatic Leadership spurred on further development and theorising in the field. The author believed that charismatic leaders possess strong traits, such as self-belief and confidence, and an ability to motivate followers towards greater performance and effectiveness through appealing to their sense of achievement, power and affiliation (House, 1977; Bass, 1985). Building on this work, Conger and Kanungo's (1987) attributional theory of charisma added that charismatic leadership involves a process of identifying the organisational environment and followers needs, formulating and articulating a clear vision for the future, and building trust and motivating followers as a means to achieving the missions and goals of the organisation.

As charismatic leadership began receiving increased scholarly attention, many other theorists began providing additional charismatic definitions and theories (Bass, 1997). A common theme within the domain of charismatic leadership was that a charismatic leader 
possessed an ability to inspire that could influence followers' attitudes and behaviours for the good of the organisation (Bryman, 1992; House, 1977).

Burns (1978), however, contended that the leader-follower relationship is bidirectional and requires reciprocal action in both party's skills when attempting to achieve a common goal. Burns (1978) and Bass (1985) extended the initial work on charisma by developing a broader concept, transformational leadership, which subsumes charisma as one of its dimensions. Den Hartog and colleagues (1997, p. 19) claimed the conceptualisation "integrates ideas from trait, style, and contingency approaches of leadership and also incorporates and builds on work of sociologists such as Weber (1947) and political scientists such as Burns (1978)". As such, Bryman (1992) referred to the origins of transformational/transactional leadership as the 'New Leadership' paradigm.

The foundations of the Full Range of Leadership theory are derived from Burns' (1978) initial description of two distinct forms of leadership - transactional and transformational - that extend previous theories as a broader and more extensive approach (Northouse, 2010; Taylor, 2014). This paradigm has been summarised by Bass as viewing:

"Leadership as either a matter of contingent reinforcement of followers by a transactional leader, or the moving of followers beyond their self-interests for the good of the group, organization [sic], or society by a transformational leader. The paradigm is sufficiently broad to provide a basis for measurement and understanding that is as universal as the concept of leadership itself" (1997, p. 130).

Additionally, the strengths of the Full Range of Leadership Theory include its ability to explain motivation (Bass, 1985); to more readily reflect leadership as opposed to simply reflecting management (Bryman, 1992; Weese, 1994); and in this case, to appeal to a range of leadership situations within other domains such as sport management (Soucie, 1994; Weese, 1994). As such, the popularity of the transformational-transactional paradigmatic stance has been unprecedented in recent leadership research since the 1980s (Bass, 1985; Yammarino \& 
Bass, 1990). Transformational leaders have been claimed as the universally most desired (Den Hartoget al., 1999; Leong \& Fischer, 2010) and effective style (Bass, 1997; Bass \& Riggio, 2006). Northouse described the style as "one of the current and most popular approaches to leadership that has been the focus of much research since the early 1980 s" (2012, p. 171), due, according to Kent \& Chelladurai (2001) to its efficacy in understanding leadership in the scope of the organisation.

The effectiveness of the New Leadership theory is within its conceptualisation of three different leadership styles: transactional, transformational and laissez-faire. Each leadership style will be discussed below in terms of their definitions, components, effectiveness and empirical backing.

\section{Transactional Leadership}

In discussing transactional-transformational theory, we will firstly outline the characteristics of transactional leadership, as many prominent researchers and scholars including Dr. Bernard Bass (1985, 1991, 1997; Bass \& Rigio, 2006; Bass \& Stogdill, 1990) and Warren Bennis (1975, 1984; Bennis \& Nanus, 1985) among others (Bryman, 1992; Yukl, 1898) contend that effective leaders display both 'exchange' and 'relationship' styles. However, transactional leadership also provides the foundation of transformational leaders; for without transactional effectiveness, transformational leadership effects may be negligible (Judge \& Piccolo, 2004). Bass, for example, has stated that, "Transformational leadership adds to the effectiveness of transactional leadership; transformational leadership does not substitute for transactional leaders" (1999, p. 21). As such, it is of foremost importance to understand the dimensions and effects of transactional leadership.

Burns (1978) initially coined the term, transactional leadership, as a summative category of existing theories of leadership that focused on the ways in which leaders rewarded or punished follower behaviour and performance (Antonakis, Avolio \& Sivasubramaniam, 
2003; Stogdill, 1974). As the name suggests, "Transactional leadership emphasizes the transaction or exchange that takes place among leaders, colleagues, and followers" (Bass \& Avolio, 1994, p. 3). A leader exhibiting this style of leadership has been characterized as "the agent at workplaces and organizations who assigns tasks to employees, delivers rewards, and promises rewards for further efforts. In addition, this type of leader sets goals, clarifies desired outcomes, provides feedback, and exchanges rewards for accomplishments" (Schmid, 2006, p. 182).

Transactional leadership has been cited as a traditional and managerial leadership style, prominent and entrenched in many business organisations (Hsu, Bell \& Cheng, 2002; Judge \& Piccolo, 2004). The leader-follower exchange is often based on bureaucratic authority and legitimacy within the organisation whereby leaders establish work standards, expectations and objectives (Hersey, Blanchard, \& Johnson, 1988; Yukl, 2002). A transactional leader orientates their relations with subordinates to manage the organisation internally. They focus on maintaining the norms and status quo of the organisation by offering followers a valued reward in exchange for their work. In turn, this approach is more suited to a stable environment with little competition (Burns, 1978; Bass et al., 2003). Leadership is viewed as being concerned with maintaining consistency by conditioning subordinates to the philosophy of the organisation.

Notwithstanding such views, according to Schein (1985), leaders have a major impact on the formation of organisational culture. Their values, beliefs and worldview govern the cultural norms, which are taught to new members. An organisational culture can "influence how people set personal and professional goals, perform tasks and administer resources to achieve them" (Lok \& Crawford, 2003, p. 323). According to this conception, transactional agreements outline the standards and norms of the organisations, disciplining and familiarizing the subordinate in accordance to the leader's expectations. Bass and Avolio 
(1994) believed that the mechanisms that leaders use to build culture are crucial in aligning the members with the vision and direction of the organisation. Consequentially, transactional leadership provides a foundation for an effective bi-directional relationship that satisfies both the leader and follower's needs.

In a similar vein, Yammarino and Bass (1990) stressed that the key to transactional leadership is the leader's ability to identify the needs and desires of the followers, and utilise these as incentives for organisational performance. Once a leader has identified the necessary subordinate action and outlines expectations, also known as role clarification, they may engage in a leader-subordinate exchange (Yukl, 1989). The leader-subordinate exchange relationship, as such, has been characterised in which the subordinate receives some reward related to lower-order needs of security, affiliation, and recognition, in return for compliance with leader expectations and organisational objectives (Doherty \& Danylchuk, 1996). Bass expanded the work by Burns, by claiming:

"The leader gets things done by making, and fulfilling, promises of recognition, pay increases and advancement for employees who perform well. By contrast, employees who do not do good work are penalized. This transaction or exchange - this promise and reward for good performance, or threat and discipline for poor performance characterizes effective leadership" (1991, p. 20).

The rewards or punishment offered by transactional leaders for adequate completion (or not) of work are derived from two types of behaviours: contingent reward and management-by-exception (Bass, 1985; Bass \& Avolio, 1994; Bass \& Stogdill, 1990). Firstly, contingent reward occurs when leaders engage in a constructive path-goal transaction of reward for performance (Bass, 1997). The goal clarification and acceptance is aimed at attaining shared goals that benefit both parties, as well as the organisation as a whole. Commitment and loyalty of followers is gathered through the "degree to which the leader sets up constructive transactions or exchanges with followers: The leader clarifies expectations 
and establishes rewards for meeting these expectations" (Judge \& Piccolo, 2004, p. 755). Moreover, they negotiate and exchange promises and resources for support of the leaders, which are derived from mutually satisfactory agreements that satisfy the follower's lower order needs along with the leader's goals (Bass, 1997).

According to the research, captured in the Multifactor Leadership Questionnaire studies, developed by Bass (1985), contingent rewards can be either tangible or intangible in nature. The most common commensurate material comes from monetary rewards such as a salary or wages; conversely, verbal praise is one of the most satisfactory and universally sought psychological responses from successful work completion. Likewise, Bass and Avolio (1994) outlined other common exchanges can lead to promotion, increased responsibilities and bonuses. It has been found that recognition of valued outcomes motivates followers to meet the leader's expected levels of performance, which are based on existing organisational structures and assigned power, and has resulted in improved organisational effectiveness in a range of settings (Stogdill, 1974; Yukl, 1989).

Despite the introduction of transformational leadership research and adoption of its findings and theoretical constructs in the identification and development of contemporary leaders, positive correlations still exist between follower's job satisfaction, extra effort and perceived leader effectiveness and contingent rewards (Bass, 1999; Lowe et al., 1996; Judge \& Piccolo, 2004). These more recent meta-analyses illustrate how contingent reward is considered, and will continue to be considered, a pertinent leadership behaviour for achieving greater organisational effectiveness.

A second dimension of transactional leadership was initially dubbed management-byexception. Bass (1999) stated this behaviour/dimension to be less effective than contingent reward as it is not as proactive in overtly recognising good work or outcomes; rather that leaders only respond to a follower's behaviours and work outcomes if they fail to adhere to 
the set expectations and benchmarks. Whilst disciplining and realigning followers who do not meet performance goals is a considered necessary response from leaders wishing to uphold the exchange relationship, this behaviour may not motivate and satisfy subordinates to the same degree as would arise from the inspirational comments of transformational leaders, or from other contingent exchanges that reward their efforts.

Bass's (1985) early work on the multifactor leadership model (based on a survey of 198 US Army field grade officers' perceptions of their superior officers behaviours) did not initially differentiate active and passive management. However, future collaborative research with fellow leadership researchers (Avolio et al., 1999; Bass \& Avolio, 1995; Bass \& Avolio, 1997; Bass \& Riggio, 2006; Yammarino \& Bass, 1990) refined the MLQ dimensions into a 9factor model to include two additional transactional leadership dimensions of active and passive management-by-exception, which are described below.

Firstly, active management-by-exception occurs when "leaders monitor followers" performance and take corrective action if deviations from standards occur. They enforce rules to avoid mistakes" (Bass, 1997, p. 134). Leaders avoid intervening and giving directions if the prescribed goals are being met (Bass, 1998). Yet the corrective nature occurs when the active supervising measures detect departures from the advised work. A corrective action may include negative feedback, re-integrating and assisting followers towards desired performance, or punishment. Consequentially, followers will have a better understanding of the roles in their job, along with their leader's expectations of them.

A second aspect of management-by-exception is when leaders are passive. This is regarded as a negative aspect of leadership, whereby the leader fails to intervene until problems become serious or compromising (Bass \& Avolio, 1997). The leader will wait passively and avoid personal contact until mistakes are brought to their attention (Bass, 1977), only acting to limit the damage from a mistake made by followers. Armstrong (1992) 
classified this as a negatively based style of transactional leadership that "could seriously impede development of personal relationships and valuable nurturing of individual leadership qualities in subordinates" (p. 33). A follower may become disheartened when the only contact that they have with their leader is due to their faults or failures, not their successes.

In summary, transactional leadership - also known as using a carrot or stick depends on the power to provide symbolic or materialistic reinforcement to subordinates on their successful completion of assigned tasks (Bass, 1997). The effective exchange, according to Bass and Avolio (1994), only occurs when the compensation is valued, or the disciplinary action is perceived as fair, by the follower in accordance with expectations. Moreover, in identifying the motivations and desires of followers, transactional leadership may only be adequate as far as the rewards equal the subordinates' efforts (Bass, 1985). Therefore, the leadership style may falter due to resource constraints. A follower will not be satisfied if the leader cannot supply them with sufficient monetary rewards, advancement opportunities, learning chances and so forth.

Transactional leadership may be effective in the short-term through its contingency reward basis (Yukl, 1989). In the long run though, Bass concluded that, "In many instances, however, such transactional leadership is a prescription for mediocrity" (1991, p. 20). Leaders are limited in their ability to access rewards equal to subordinates' efforts. Moreover, management-by-exception focuses too heavily on mistakes, which may demotivate followers by focusing on their faults and incompetence; additionally, leaders who only intervene when problems become chronic may have left it too late to fix.

At this point, something more than transactional leadership is needed to motivate and inspire workers' and satisfy their higher order needs. As Burns (1978) stated, the transactional style consists of a relationship that goes no farther than the exchange, and that the leader and follower may go their separate ways after the transaction. In this regard, 
personal relationships are less important than whether or not goals have been met. Inevitably, leaders will have to employ different behaviours in order to allow followers to attain their higher order needs, and in turn, achieve greater organisational performance and effectiveness.

\section{Transformational Leadership}

Leadership research in the 1980s saw more scholars become interested in the transformation of organisational standards, cultures and relations. Globalisation of industries through technological innovations caused many leaders to modify their existing system or culture in order to survive (Yukl, 1989). According to Burns (1978) and Bass (1985), the transactional leadership style, which operates within current practices, was perceived as an insufficient behaviour for reacting to, shaping and creating the environment organisations wish to compete in.

In order to respond to the changing environment, many considered that a new approach to leadership was required. One such supporter of this belief was Dr. Bernard Bass, who asserted that,

"The recent shift in modern industry away from an emphasis on organizational hierarchies and strict adherence to a chain of command to a 'team' approach to management has significantly altered our thinking in the area of leadership. What is interesting to me is that the concept of shared leadership was proposed and discussed in the 1940s and 1950s, although industry didn't embrace the concept with much enthusiasm until recently" (Weese, 1994, p. 182).

James MacGregor Burns instigated research focusing on how leadership must change to deal with organisational reforms through his formative work in the book Leadership (1978). His concentration was on addressing reform or restructuring by focusing on empowerment, shared leadership and social influence through a new leadership model. It was posited that this shared leadership may exhibit a cascading outcome known as the "falling 
dominoes effect', whereby followers assume leadership roles and seek to imitate transformational leaders they respect (Bass \& Avolio, 1994). Bass and colleagues (1987) found support for this effect in the New Zealand public sector.

As such, a transformational leader was conceived as the most effective leadership style for the contemporary organisation (Bass \& Riggio, 2006). The efficacy of his work is still apparent twenty years on; whereby prominent scholars assert that transformational leadership is the primary model for the aforementioned characteristics (Avolio \& Bass, 1999; Bass, 1997; Bass \& Avolio, 1994; Leithwood \& Jantzi, 2000).

Transformational leadership draws inspiration from prior theorising on leadership definitions, traits, behaviours and contingencies (Burns, 1978; Bass, 1985). For example, Warren Bennis believed one of the central tenets of effective leadership is the ability to formulate a vision, and implement it $(1959 ; 1984 ; 2001)$. Vision is defined as "the capacity to create and communicate a compelling picture of a desired state of affairs, impart clarity to this vision, and induce commitment to it" (Bennis \& Nanus, 1985, p. 181). Burns (1978) and Bass (1985) argued that a vision of the future is something that all transformational leaders communicate to their followers, and inspire these subordinates to reach. Secondly, the effective transformational leader will possess desirable traits, which act to complement and reinforce their behaviours. For instance, honesty, trust and integrity are necessary when gathering support from subordinates towards the visionary state (Bennis, 1984; Kirkpatrick \& Locke, 1994).

Furthermore, a meta-analysis of over 30 years of empirical research found the notion of self-confidence to have validity in its ability to differentiate effective and non-effective leadership (Judge et al., 2002). Such self-confidence is a pre-requisite for authentic charismatic leaders. Finally, aspects of behaviours and situational theories were then extracted and introduced as relationship-based actions that encompassed inspirational motivation and 
individualised consideration (Avolio et al., 1999); also, Bass (1985) acknowledged that different behaviours are required in accordance to the leadership preferences of subordinates, the organisational setting or desired follower outcomes.

All of these aspects can be seen as interwoven within Burns' (1978) assertion that a transformational leader in nature will engage in the higher order needs of individuals or groups. According to Maslow's (1954) hierarchy of needs, the transactional leader can only satisfy the basic needs of the subordinate, however, a leader who is transformational will inspire and empower followers to go beyond self-actualisation (Bass, 1999). These leaders strive to raise "the consciousness of followers by appealing to higher ideals and moral values such as liberty, justice, equality, peace, and humanitarianism, not to baser emotions such as fear, greed, jealousy, or hatred" (Yukl, 1989, p. 210). In doing so, the follower will be able to reach a greater level of motivation - and in turn performance - along with a sense of commitment towards the common goal (Shamir, House \& Arthur, 1993).

The inspiration and empowerment imparted within the organisation may occur at both the macro and micro levels. Leaders can influence individuals (micro level) through pleasing the aforementioned values and acknowledging the personal desires and needs. Likewise, transformational behaviours alter existing structure and reform practices as a response to changing environments in which their organisations compete (Bass, 1999; Yukl, 1989). Within a contemporary and ever-evolving industry, a transformational leader can mobilize their power to change social systems and cultures (Burns, 1978), which Bass (1998) believes is necessary for their survival. Such leaders will understand and influence the values, norms and beliefs of the followers, allowing them to transform the true needs of the subordinates (Imran, Zahoor \& Zaher, 2012; Parry \& Proctor-Thomson, 2003; Schein, 1992). In turn, transformational leadership, occurring at the macro-level, may link to enhanced organisational effectiveness (Weese, 1996). 
As the popularity of the transformational leadership model grew, so too did the need to operationalise its dimensions and test its validity. Dr. Bernard Bass was one recognised as presenting "a formal theory of transformational leadership as well as models and measurements of its factors of leadership behavior [sic]" (Bass \& Avolio, 1994, p. 2). Bass had disagreed with Burns' theorising, predominantly in relation to the posited continuum. Burns (1978) believed that transactional and transformational leadership are distinct styles at opposite ends of a continuum. Conversely, "Bass views transformational and transactional leadership as distinct but not mutually exclusive" (Judge \& Piccolo, 2004, p. 176). In other words, Bass considered that a leader could exhibit both transactional and transformational dimensions in accordance with the needs of the situation, and of the subordinate.

Bass (1985) believed that transformational leadership is in fact related to transactional behaviours. After a decade of research, Bass (1999) commented that "the best leaders are both transformational and transactional" (p. 21). Another influential scholar, Bruce Avolio, agreed with this viewpoint suggesting that without the foundation of transactional leadership, transformational effects may not be possible (Avolio, 1999). When the two researchers collaborated, they contended that transformational leaders could supplement the efficacy of exchanges (Bass \& Avolio, 1994) in as much as "consistent honouring of transactional agreements builds trust, dependability, and perceptions of consistency with leaders by followers" (Bass, 1998, p. 11). However, Yammarino and Bass claim the transformational "has a greater impact on outcomes that count" (1990, p. 155).

Bass (1985) believed such leaders influence subordinates to aspire to higher levels of performance for the good of the organisation. Bryman (1992) reinforced Bass's position that transformational leadership has a significant relationship with organisational outcomes. $\mathrm{He}$ stated that these leaders can influence subordinates in three ways: (a) to embrace a greater 
vision of the organisation, (b) to exert greater effort in the pursuit of higher order needs, and (c) to perform beyond expectations.

According to Bass's (1985) model, transformational leaders are able to achieve these outcomes through any combination of its dimensions. The constructs that govern the theory have been revised and modified through various developments and applications of the MLQ instruments. The original MLQ was developed from a small study of 70 senior executives' responses to questions regarding what type of people elevate their consciousness and motivation on Maslow's hierarchy of needs (Bass, 1985). The survey yielded 141 statements that were then sorted by 11 trained judges into constructs that provided researchers with nascent operationalised dimensions for empirical study.

Initially, Bass and Avolio (1990) conceived four transformational leadership dimensions. However, scholars in the late 90's argued that the charismatic component had not sufficiently been altered from past theories (Avolio et al., 1999; Bass, 1997). In response to the criticism, Avolio and Bass (2003) revised idealised influence (charisma) into attributed and behaviour aspects. In the new millennium, the theory has settled on an expanded set of five transformational factors, which include idealised influence (attributed), idealised influence (behaviour), inspirational motivation, intellectual stimulation and individualised consideration. The dimensions are briefly described below.

Charismatic leadership as defined by Bass (1985) was a crucial pillar of transformational leadership theory. Although Bass drew inspiration from previous theories of charisma and charismatic behaviour, his idealised influence dimensions differ in distinct ways. For instance, shared leadership and subordinate empowerment are integral to transformational leadership whereas charismatic leaders seek to maintain sole power (Manning, 2012). Additionally, the falling dominoes effect illustrates how transformational leadership can occur at any level of the organisation whereas charismatic leaders are usually 
only in top administration (Yukl, 1989). In spite of the differences idealised influence is very similar as "leaders engender trust from and serve as role models for followers" (Bass et al., 1996, p. 14). Moreover, Bass (1997) believed idealised influence occurs when:

"Leaders display conviction; emphasize trust; take stands on difficult issues; present their most important values; and emphasize the importance of purpose, commitment, and the ethical consequences of decisions. Such leaders are admired as role models generating pride, loyalty, confidence, and alignment around a shared purpose" (p. 133).

Bass's early (1997) definition entails both the attributes and behaviours of idealised influence; however, the former is mainly concerned with the inherent aspects of the leader, such as confidence and power, whereas the behaviours are the application of these characteristics (Bass, 1999; McCarley et al., 2014). By setting high standards and expectations, followers respect the leader and want to follow and emulate these people (Bass et al., 2003).

The second dimension also acts to gather support around the leader's vision and performance expectations. Inspirational motivation is the ability of the leader to emotionally inspire, provide encouragement and meaning towards the follower's organisational efforts (Atmojo, 2012; Bass, 1997). Resultantly, followers become committed to and part of the shared vision of the organisation, working with extra exuberance and dedication (Northouse, 2012). The leader will challenge the basic expectations by "providing significance and challenge to the followers' work and by promoting team spirit, enthusiasm, and confidence" (Manning, 2012, p. 46). Open communication from the leader is also considered to be vital in order to gather trust and support. By operating with transparency, the followers will more readily understand the needs of the leader (and organisation) and meaning of their roles (Bass \& Rigio, 2006). As both constructs are tied to the vision of the organisation, inspirational motivation can be seen as a subset of idealised influence according to Avolio and Bass 
(2004). Yet it is still a distinct dimension, as one does not have to possess idealised influence to be inspirational.

For leaders to transform and revitalise an organisation, they must question old beliefs, practices and assumptions. Bass (1997), for example, believes the ability to stimulate followers in to viewing their practices and those of the organisation through a new lens are critical for change. In the early decades of the new millennium, the efficacy of intellectual stimulation is still considered a necessary component ensuring competitive advantage through responsive change (Jandaghi et al., 2009; McCarley et al., 2014). Broadly speaking, intellectual stimulation occurs when leaders seek "to bring forth the expert knowledge of the members of the organization [sic], thinking is stimulated at all levels about the organization's [sic] objectives and means to meet them" (Bass, 2000, p. 26). A transformational leader achieves these outcomes by encouraging followers to become more innovative and creative through questioning old traditions, beliefs and assumptions (Bass, 1997; 1999).

The leader supports these desired behaviours by creating an environment where there is no ridicule or public criticism of followers' mistakes, or of new ways of doing things. "New ideas and creative solutions to problems are solicited from followers, who are included in the process of addressing problems and finding solutions" (Bass et al., 2003, p. 208). As such, it is suggested that leaders can create a supportive climate where subordinates feel free to express themselves.

Finally, individualised consideration is a fifth dimension of transformational leadership, focussing on "development and mentoring of followers" and attending to their “individual needs" (Eagly et al., 2003, p. 571). The leader will act as a mentor or coach to the follower, engaging in a personal relationship with each individual (Judge \& Piccolo, 2004). When assigning tasks or engaging in exchanges, the leader will consider their individual needs, aspirations and abilities (Bass, 1997), delegating jobs as opportunities for growth 
(Bass, 1998). In summary, many scholars such as Atmojo (2012) contend that transformational leaders who display this behaviour encourage greater productivity, as followers feel valued and satisfied with their contributions.

The five dimensions of transformational leadership are often referred to as the '5 I's', which many scholars believe possess distinct advantages over previous theorising. Most pertinently, transformational leadership has maintained its prominence over time, with, for example, Stewart's (2006) review of leadership in education identifies transformational leadership as an effective leadership style for school administrators. Similar reviews in sports management (Soucie, 1994) and Australasian sports organisations (Gilbertson, 2000) assert that the notion of transformational leadership can provide reliable and valid framework for understanding effective management.

Northouse's (2012) Leadership: Theory and Practice outlines the standing of transformational leadership theory/model. He states that (1) transformational leadership has been researched extensively, constituting a third of articles on Leadership Quarterly from 1990-2000; (2) the model focuses on the leader-follower relationship, thereby examining the desires and outcomes for the follower and not solely focusing on the leader; (3) the broad theory has augmented trait, behavioural, situational, servant, and charismatic leadership theories; and (4) transformational leadership has surpassed previous theories due to its organisational practicality. Judge and Piccolo (2004) outline the substantial empirical evidence proving that it is not simply abstract and theoretical.

Although the strengths of transformational leadership theory are apparent and its popularity amongst scholarly researchers has been unprecedented, critics have still drawn attention to its flaws. The methodological rigour has been questioned by a number of scholars including Northouse (2012) who specifically discussed how the transformational dimensions often correlate highly with each other. Judge and Piccolo's meta-analysis on the validity of 
the Full Range Model also concluded "transformational and transactional leadership are so highly related that it makes it difficult to separate their unique effects" (2004, p. 765). Consequentially, continuing use of the existing MLQ instrument may not provide sufficient validity in its ability to differentiate leadership behaviours, let alone claims towards organisational effectiveness (Hartog et al., 1997; Meunjohn \& Armstrong, 2008).

Another methodological concern involves data gathering measures. Bryman recently claimed, "Leadership research has been and almost certainly still is a field that is dominated by a single kind of data gathering instrument - the self-administered questionnaire" $(2004, \mathrm{p}$. 731). Most notably the MLQ instrument has received criticism for bias and exaggeration by respondents, amplified by the difficult task of retrospectively rating leadership behaviours (Yukl, 1989). "As the limitations of questionnaire-correlational research have become more apparent, some leadership researchers have turned to qualitative, descriptive methods such as observation, interviews, and intensive case studies" (Yukl, 1989, p. 278). Bryman's (2004) review of qualitative leadership research illustrates the fields' response to this concern with a growing number of qualitative studies on transformational leadership. Additionally, Bryman and colleagues (1996) have also argued that qualitative research can provide a deeper understanding of context and other moderating variables.

In regards to the theoretical assumptions of transformational leadership, Tourish's (2013) book, The dark side of transformational leadership: A critical perspective, questions the excess agency of leaders along with the belief that followers are positioned as passive, compliant beings. The excess agency has been considered potentially detrimental to the organisation as such leaders may operate and make decisions without having to seek the consent of others. Bass and Riggio (2006) share this apprehension whereby they outline the potential destructive power of leaders who lack a strong moral foundation. Wilson's (2015) review of Tourish's work supports these concerns regarding power, reasoning that leadership 
assumptions may have be 'reinvisioned'. Likewise, de Villiers (2014) recommends that leaders (in the New Zealand context) should read this book to question their practices and ultimately improve their effectiveness.

Lastly, Bryman (1992) questioned whether or not some transformational leadership dimensions could really be taught or developed. He believed that many leaders had a predisposition and traits were congenital/innate as opposed to learned. For example, debate has emerged over whether or not it is possible to develop traits such as extraversion. In this instance Bennis and Nanus (1985) claim it to be an innate trait, however, Mason and colleagues (2014) found development courses could in fact improve self-efficacy, which may include traits such as extraversion.

\section{Laissez-Faire Leadership}

A conceptual polar opposite to transformational leadership on the Full Range of Leadership Model continuum is the non-leadership aspect, laissez-faire. Bass believed such leaders are neither transactional nor transformational, rather, there is no involvement with colleagues, and subordinates primarily function on their own (1985). As such, it may be thought that passive leaders avoid accepting their responsibilities - for example, through absenteeism, or in regards to defining agreements, expectations and standards (Bass et al., 2003). They may also fail to follow up the concerns and requests from their subordinates (Bass, 1997). Possibly the most detrimental aspect of laissez-faire leadership occurs when such leaders resist expressing their views on important issues (Avolio, 1999). This is in direct contrast to widespread belief that leaders (regardless of style) must provide vision and direction for their followers.

\section{Empirical research of Full Range of Leadership}

The full range model of leadership has been empirically tested across a wide range of environments and settings. Most notably, the Multifactor Leadership Questionnaire (MLQ) 
instrument has underpinned this research, which "implies that every leader displays a frequency of both the transactional and transformational factors, but each leader's profile involves more of one and less of the other" (Bass, 1999, p. 11). In order to test the validity and reliability of the model the MLQ has received rigorous reviews.

Over a decade after the preliminary conceptions of transformational leadership Avolio and colleagues (1999) sought to re-examine the dimensions of the model. The authors' review of 14 studies, comprising 3,786 respondents concluded that the nine-factor model used in this current research study - is most desirable in the MLQ literature. More recently, Meunjohn and Armstrong's (2008) also provided support for the nine-factor model in their evaluation of the structural validity of the MLQ instrument. Despite relatively high interconnectedness of leadership dimensions (Bass, 1997b), the forty-five questions in the MLQ 5X self-rater instrument have been found to provide appropriate measures of each of the nine factor dimensions.

In addition to testing the structural validity of the MLQ survey instrument, researchers have tested its external validity in a range of settings, attempting to generalise findings. Bass (1997) stated, "Many situational contingencies may be posed as variform functional universals that raise or lower the means, variances, and correlations with outcomes" (Bass, 1997, p. 137). As such, studies have been aimed at determining what demographics and features of leaders correlate with the leadership dimensions; whether transformational leaders can be developed; which leadership behaviours are more prevalent in different industries; which moderating variables and contingencies may influence the leader-follower behaviours; whether or not the transformational leadership theory has universal relevance, and which leadership behaviours are more pertinent to certain cultures.

Leader-centric studies have pursued understanding of the innate variables that may determine a propensity to adopt certain styles of leadership. Despite Bass (1985), and Bass 
and Avolio (1990) proposing that transformational leadership is a developed behaviour, leader characteristics such as gender, age, experience and organisational position may also predicate behaviours (Avolio, 1999). It is considered important that these are recognised as important antecedents, or not, as they may significantly impact a range of organisational outcomes.

Previous research on the relationship between gender and leadership in organisational settings led Bass, Avolio and Atwater (1996) to hypothesise that gender differences will occur in the surfacing frequencies of certain leadership dimensions. Testing this hypothesis has revealed mixed results, however, the general consensus is that women tend to display somewhat more transformational behaviours than men (Bass, 1999; Doherty, 1997). Similarly, a meta-analysis of leadership and gender studies between 1985 and 2000 revealed small gender differences (Eagly et al., 2003). Female leaders' greater frequency of transformational behaviours may be attributed to gender roles and norms, and glass ceilings, producing highly skilled female leaders, as discussed by Martin (2015).

Scholars have also been interested in the relationship between age and experience with leadership behaviours. "Just as with gender, bias in perception exists with age and leadership" (Martin, 2015, p. 337). Generational differences have led some leadership theorists to assert that older leaders would be more accustomed to transactional behaviours, as these behaviours dominated industry prior to Bass's (1985) transformational leadership theory. Doherty (1997) claimed younger managers may be more comfortable with transformational leadership, whereas those middle-aged and elderly leaders may favour their historical styles. Yet without significant experience in the role, leaders may seek stability and follower understanding through exchange relationships (Avolio, 1999). Conversely, Martin's (2015) recent exploration of literature has suggested that the age difference in the leader-follower relationship means that the leaders older than their subordinates may be more able to use 
transformational leadership. Nevertheless, legitimacy for both directional relationships has been shown illustrating the need for further research.

Innate leader characteristics may impact the frequency of transformational behaviours as shown above. Nevertheless, transformational leadership theory was conceived under the assumption that like the behavioural approaches, transformational leadership too can be taught and developed (Burns, 1978; Bass, 1985). Most recently de Vries et al's (2009) longitudinal study, and Mason's (2014) emphasis on psychological development, found support for transformational leadership development courses. These courses can also have significant positive impacts on the attitudinal and financial outcomes within the organisation (Barling, Weber, \& Kelloway, 1996). The implications for organisations are that it may be more beneficial to develop leaders internally as they "can reap huge benefits by helping good managers become great" (Zenger, 2013, p. 20).

Many leader characteristics may moderate their behaviours; similarly, widening the scope of inquiry to the organisational- and environmental-level reveals similar discrepancies. Firstly, transactional behaviours may be more suited to stable settings, whereas transformational behaviours may be required to adapt the organisation's vision and purpose in turbulent environments (Antonakis et al., 2003; Burns, 1978; Bass et al., 2003; Gunderson et al., 2012). Secondly, Bass (1985) posited that private organisations allow more transformational leaders to emerge when compared to mechanistic public organisations. Despite this, Lowe and colleagues' meta-analysis of research from various countries found a relationship, contrary to their expectations (1996). As research has produced contradictory results, one may not be able to generalise transformational behaviours in accordance to the organisation's sector.

Although mixed results have appeared at the organisational level, transformational leadership has been endorsed as a universally applicable theory (Bass, 1985, 1997; Den 
Hartog et al., 1999; Leong \& Fischer, 2010; Yukl, 1989). Bass affirms the theory can offer utility to all nations, however, "the linkages among concepts may strengthen or weaken as one moves from on culture to another" (1997, p. 132). To test cultural differences, Leong and Fischer (2010) analysed MLQ research between 1985 and 2006 from 18 nations. The authors found that cultural differences are marked, explaining up to $50 \%$ of the variability in leadership behaviours. Moreover, societies that emphasize egalitarianism, and are lower in power distance, were consistently associated with higher frequencies of transformational behaviours, as with collectivistic cultures (Ramachandaran \& Krishnan, 2009). These findings illustrate the profound cultural differences acting to shape desirable and appropriate leadership styles.

Understanding the relationship between leadership and culture is crucial to organisational performance (Imran, Zahoor \& Zaheer, 2012; Schein, 1992; Weese, 1996). Ramachandaran and Krishna argued "leaders will not be able to understand the true needs of followers if they do not understand their values, norms, and beliefs" (2009, p. 30). Bass (1985) and Bass and Riggio (2006) found that transformational leadership was the most preferred and favourable style for interacting with and satisfying followers. Bass then applied his theory to determine a hierarchy of correlations among leadership styles and outcomes in effectiveness (1997). Results supported his belief that transformational behaviours sit atop a pyramid of behaviours, followed by contingent reward, management-by-exception and finally laissez-faire behaviours.

\section{Organisational Effectiveness}

Many studies have examined organisational effectiveness, and the effectiveness of leaders in impacting organisational performance. In this study, organisational effectiveness is regarded as the dependent variable when related to transformational leadership. However, like leadership, as a multi-dimensional construct, it is hard to define and measure 
(Chelladurai, 1987). Slack (1997) explains "despite the problems associated with the ideas of organisational effectiveness, creating an effective organisation is in many ways the central task of the sport manager" (p. 19). The behaviours of the leader will have an impact on many micro and macro outcomes in the organisation.

Authors such as Cameron (1978) have outlined the numerous definitions along with the four main ways to assess organisational effectiveness: goals, process, systems and multiple constituency perspectives. The goals approach states an organisation can be effective if it can achieve its specific goals; the process perspective declares effectiveness in regards to internal processes and daily operations functioning smoothly; and effective organisations acquire scarce and valued resources from the environment according to the systems approach (Cameron, 1978; Soucie, 1994).

In the late 1990s, Weese claimed that the goals approach was the most commonly used approach to measure organisational effectiveness in sport management (1997). However, limitations and weaknesses with the goals, processes and systems approaches led to extensive recommendations for researchers of sport organisations to adopt the multiple constituency approach (Hsu et al., 2002; Soucie, 1994; Weese (1994). According to Yukl's (1989) review of managerial leadership, this approach is most appropriate as it synthesizes the other three perspectives - goals, processes, and systems resources - analysing organisational effectiveness using a variety of criteria reflecting the attitudes of different stakeholders of the organisation. For instance, it can encompass student participation and success. In addition, coaches' and school staff satisfaction and effort can be classified as a measurement of school sports coordinator effectiveness.

The multiple constituency approach has been employed to examine leadership behaviours in a variety of managerial and organisational contexts to better understand its relationship with effectiveness. Most notably the MLQ instrument has been used within this approach as it 
focuses attention on how perceived leader effectiveness, subordinates' job satisfaction and extra effort may contribute to greater organisational effectiveness (Bass \& Avolio, 1997). Bass's inspiration for the inclusion of these organisational outcomes in the MLQ instrument was drawn from findings in his book Leadership: Performance beyond expectations (1985), whereby greater effectiveness was achieved when leaders operationalize any combination of the five transformational behaviours and some transactional dimensions.

After 30 years of empirical research (Bass, 1994; Bass \& Avolio, 1994; Bass et al., 2003; Bass \& Rigio, 2006; Northouse, 2012) and meta-analyses (DeGroot, Kiker \& Cross, 2000; Lowe et al., 1996; Judge \& Piccolo, 2004; Wang, Oh, Courtright \& Coulbert, 2011), scholars have shown that transformational and transactional leadership may positively predict a range of organisational outcomes that contribute to greater performance and effectiveness.

The significance of the relationships of transformational leadership with effectiveness is that it is not limited to simple leader-follower exchanges; it has efficacy at the individual, group and organisational level (Bass, 1999; Weese, 1996; Wang et al., 2011). Accordingly, leaders can exert their influence at the micro level while simultaneously creating a culture of empowerment and shared leadership. Both effects can act to produce favourable outcomes for a number of stakeholders; for followers these can include greater job satisfaction, extra effort and perceived leader effectiveness.

\section{Job Satisfaction}

After reviewing the empirical and theoretical applications of the transformational leadership theory, Bass concluded that, "Transformational leadership, which fosters autonomy and challenging work, became increasingly important to followers' job satisfaction" (Bass, 1999, p. 10). Moreover, an employee's satisfaction with their job occurs only a number of levels. Locke's research, The nature and causes of job satisfaction (1976), 
discusses the multi-faceted construct of job satisfaction to include satisfaction with fellow individuals in the organisations and with the nature of their work. This can occur externally or internally, and different leadership styles will engender different working environments and directly affect the job satisfaction of the employees (Bass, 1985).

Academics and researchers have investigated the possible relationships between leadership styles and job satisfaction in a number of settings. Early research utilising the MLQ's ability to quantify leadership styles and organisational outcomes, for example, job satisfaction, has provided general support for a positive relationship with transformational leadership. Studies across a number of different nations have found that transformational leadership may predict satisfaction with Indonesian (Atmojo, 2012) and Libyan organisations (Shurbagi, 2014), and the Malaysian public sector (Voon, Lo, Ngui, \& Ayob, 2011). This indicates that the relationship may not be restricted to Western organisations.

Podsakoff and colleagues (1996) found general support for a relationship between leadership and job satisfaction, their findings indicating that leadership scores accounted for approximately $71 \%$ variance in follower's job satisfaction. However, the transformational leadership model explained less variance in their research than the substitutes of leadership model. Further critique of the transformational theory has been found in the educational sector, whereby Darvish and Shirazi Pour (2013) found that transactional behaviours had a greater impact on the satisfaction of 312 private sector college teachers.

Support for the transformational theory has been established in Rizi and colleague's (2013) study on physical education employees, finding transformational leaders to be a key determinant of job satisfaction. By contrast, Wallace and Weese (1995) have found there to be no significant differences in employee job satisfaction for different levels of transformational leadership. However, the work of Lok \& Crawford $(2002 ; 2004)$ suggests that this may be 
due to the antecedents of commitment and effects of culture, acting to affect the relationship between leadership and satisfaction.

Despite a majority of research findings that suggest a relationship between transformational leadership and job satisfaction, the mixed or ambiguous results that have surfaced in both sporting and educational settings mean that this setting may require different leadership than the private sector. As such, further research is required.

\section{Extra Effort}

The second organisational outcome explored through the MLQ is extra effort. The key pillar of transformational leadership is the ability to inspire followers to transcend their self-interests for those of the group, and in doing so, exert a greater effort in their pursuit of success (Bass, 1985; Bass \& Avolio, 1994). As such, scholars have hypothesized that leaders who exhibit transformational leadership behaviours will induce their followers to exert extra effort as a result.

According to findings from MLQ studies, a leader can induce extra effort in their followers by getting them 'to do more than they expected to do', increasing their 'desire to succeed', or 'increasing their willingness to try harder' (Bass \& Avolio, 1997). What's more, Atmojo (2012) claimed extra effort includes motivation, work ethics, work attendance and work planning. All of these are intended to culminate in a follower-base that contributes to greater organisational effectiveness.

In addition, the assumption that "followers feel trust and respect toward the leader and they are motivated to do more than they are expected to do" (Yukl, 1989, p. 272), has provided academic support of the correlation between extra effort and transformational dimensions (Bass, 1985; 1997; 1998; 1999; Bass et al., 2003). In subsequent studies, this relationship was also found to be applicable to physical education employees (Rizi et al., 2013) and the leaders of tertiary school sporting programmes (Doherty \& Danlychuk, 1996; 
Doherty, 1997). These results provide general support of Bass's findings that the effectiveness of leaders to inspire extra effort may be determined by their ability to adopt transformational behaviours.

In spite of the apparent relationship between transformational leadership and extra effort, rigorous reviews of literature have provided contrary evidence. For instance, Judge and Piccolo's (2004) meta-analysis included 87 studies from journal articles and dissertations, reporting a total of 626 correlations. In reviewing these correlations, the authors hypothesized that transformational leadership spurs extra effort and higher performance at the group level. Interestingly, their meta-analytical findings indicate the relationship between transformational leadership and contingent reward was not significant. Similarly, DeGroot and colleagues (2000) conducted a meta-analysis of organisational outcomes related to charismatic leadership, finding no support for the hypothesis that charismatic leaders will engender extra effort amongst followers.

With parallels to findings on job satisfaction, support for the positive organisational outcomes of transformational leadership has been inconclusive. The situational and subordinate contingencies for any given organisation may vary drastically, meaning that different leadership behaviours may elicit a range of low to high efforts from followers. Further research is required to gather a greater understanding of how leadership behaviours may affect extra effort, and under what circumstances.

\section{Perceived Leader Effectiveness}

A third organisational outcome measured through the MLQ instrument is perceived organisational effectiveness. Accordingly, a leader of an effective organisational will meet subordinates' job-related needs, represent their group to higher authorities, and meet the requirements of the organisation as a whole (Bass \& Avolio, 1997). The perceived effectiveness of transformational leaders occurs at multiple levels of the organisation (Wang 
et al., 2011) supporting the notions of empowerment and shared leadership that govern transformational leadership theory indicating that leaders who display transformational tendencies may be more effective than transactional behaviours.

The benefits of transformational leadership appear to be relevant to such leaders' effectiveness. A quantitative review of charismatic leadership (a subsumed dimension of transformational theory) revealed a strong and significant correlation coefficient of 0.79 with perceived leader effectiveness (Fuller et al., 1996). Within the business setting, Judge and Piccolo's (2004) review of the Full Range of Leadership Theory indicated a similar relationship. Educational research, such as tertiary school sport programmes (Doherty \& Danlychuk, 1996; Doherty, 1997), has revealed transformational behaviours to be more highly correlated than transactional factors with perceived leader effectiveness. Contrasting these findings, Burton and Peachey (2009) discovered that transformational leadership was not more highly correlated with effectiveness than transactional leadership.

Although the dimensions of leader effectiveness (Bass \& Avolio, 1994) encompass the individual-, team- and organisational-level needs, they are relatively vague and may include many organisational outcomes. Confounding this situation, the multiple constituency approach stipulates the ability of the leader to achieve these ends will vary according to the vision of the organisation and the diverse needs of stakeholders.

It is not surprising then, that perceived effectiveness of leaders is highly contextualised to their subordinates and environmental conditions, and that the nature of relationship between leadership behaviour and perceived leader effectiveness may vary across industries and cultures (Fuller et al., 1996).

Moreover, leader effectiveness may be affected by a variety of factors and constructs. The literature suggests that many such constructs are interrelated, and may contribute to overall perceived leader effectiveness, either independently or in tandem with one another 
(Bass \& Avolio, 1994). For example, organisational citizenship behaviours, or "those behaviors [sic] which are not formally prescribed, but yet are desired by an organization [sic]" have been linked with organisational outcomes such as commitment, satisfaction, and effectiveness (Schnake, 1991, p. 736). Further subsets of perceived leader effectiveness may include innovation, cohesion and motivation, and follower performance.

The ability of a leader to promote innovation throughout their organisation often enhances competitive advantages and quality of products or services. "By developing cultures that foster innovation, non-profit managers can help their organizations become more responsible to the changes in the external environment and become more effective" (Jaskyte, 2004, p. 164). This may help non-profit managers to overcome turbulent and competitive environments, which often jeopardise their survival.

Scholarly research has suggested that transformational leader behaviours may be most appropriate for fostering innovation. As transformational leadership theory emphasizes the role of empowerment, Jung and colleagues (2003) explored its association with innovation, finding positive relations. For example, if followers see themselves as significant and a significant part of the community they may feel more inclined to participate in decisionmaking processes. The capacity of the leader to promote debate among followers - possibly through intellectual stimulation - has also been shown to affect innovation (Boerner, Eisenbeiss, \& Grisser, 2007). Through transformational behaviours, subordinates are not only empowered to be more innovative. Tafvelin and colleagues' longitudinal study (2011) found that the effects of transformational leaders in creating a culture for innovation might mediate follower well-being. Workers who experience positive emotions, elicited by the leader, may also experience greater role clarity and meaningfulness of their work, and in turn, be more committed to the vision of the leader. 
Organisational commitment is defined as "The degree to which an employee identifies with a particular organization [sic] and its goals, and wishes to maintain membership in the organization [sic]" (Atmojo, 2012, p. 116). According to Meyer and Allen (1991), one can be committed through an emotional attachment or involvement (affective); by perceiving the cost of leaving as too high (continuance); or through an obligation to remain in the organisation (normative).

Transformational leadership dimensions have been shown to enhance commitment (Bass, 2000), for example, through vision and developed trust from followers (Atmojo, 2012). In the context of this study, similar results have been found where collectivistic cultures (Ramachandaran \& Krishnan, 2009), such as some Australasian organisations, are more committed to their leaders (Lok \& Crawford, 2004); the directors of school sporting programmes, who exhibit transformational behaviours, have more committed coaches (Kent \& Chelladurai, 2000); and transformational leadership is more effective in contemporary and unstable environments by significantly influencing follower's commitment (Tyssen, Wald, \& Heidenreich, 2013).

Lastly, empirical research from organisations with similar traits to secondary school sports programmes provide results that lead one to hypothesise that transformational leadership behaviours are important to quality provision. Small organisations can experience greater profitability and success through transformational leaders (Valdiserri \& Wilson, 2010). In addition, dynamic environments (Gunderson, Hellesoy, \& Raeder, 2012) and temporary teams (Jenewein \& Schmitz, 2007) may be more effective with transformational leaders.

"Since its inception, research has demonstrated the utility of transformational leadership for increasing organizational [sic] satisfaction, commitment and effectiveness" (Bass, 2000, p. 21). The relationship between transformational leadership and follower job 
satisfaction, extra effort and perceived leader effectiveness has also found general support in meta-analyses (DeGroot et al., 2000; Lowe et al., 1996; Judge \& Piccolo, 2004). As previous research studies within similar organisations (Doherty, 1997; Singer, 1985; Stewart, 2006) has illustrated, significant relationships exist between transformational leadership behaviours and the three mediating variables that contribute to organisational effectiveness.

Nevertheless, ambiguous, equivocal and contradictory findings have been uncovered in both the educational and sporting contexts, weakening generalizability and validity. The ambiguous results "suggest that more research is needed to test this theory in sport settings" (Aminuddin, 1998, p. 171).

\section{Leadership in Sport, Schools and New Zealand}

Sport management has seen a shift in focus to become more business-like and to incorporate private sector practices in the way they manage their employees (Chelladurai, 2001). In response to the changing environment in which they operate, sport managers are seeking to adopt and promote new leadership styles to ensure their organisations remain effective.

In light of increased pressure of professionalization, managerialism and accountability in sport, Weese stated that,

"Administering sport programs in the 1990s is a challenging exercise. Economic realities, coupled with the many a varied entities competing for limited resources, make the need for effective leadership even more acute. The transformational leadership literature offers valuable information to the practicing sport manager relative to focusing on a working force and inspiring people within it toward the attainment of organization's goals” (1995, p. 187).

To address the need for leaders to adopt a 'team' and more 'business-like' approach, transformational leadership may be employed as it has the potential to promote employee engagement with the organisation's vision, while also encouraging followers to exert extra 
effort and up skilling (Bass \& Rigio, 2006). Research from Sharpe (2006) has shown that financial and structural capacity are often lacking in grassroots sporting organisations. However, social capital (the interactions among members) can be utilized to overcome these matters. Doherty's (1998) review of organisational behaviour in sport reinforces the importance of the 'New Leadership' approach for favourable individual and group outcomes.

Bass believes his transformational leadership theory/model to be applicable to such contexts, especially given the shift to a team approach of management (Weese, 1994). Aminuddin considered "sports administrators may be able to motivate subordinates to achieve higher goals and to do more for the organization with fewer resources" (1998, p. 171). This claim has been supported by a number of key authors in sport management (Chelladurai, 2001; Gilbertson, Blyde, Gianotti, \& Gilbertson, 2000; Parry \& Proctor-Thomson, 2003; Slack \& Parent, 2006; Weese, 1994).

In the late 1980s, Paton (1987) believed that leadership has become the most popular subject within the field of sport management, largely thanks to the application of theories and instruments borrowed from other social sciences fields. Subsequent studies related to the New Leadership style rose to prominence in sport management research in the 1990s (Armstrong, 1992; Weese, 1995; Doherty, 1997; Doherty \& Danylchuk, 1996; Ristow et al., 1999). Scholarly research has maintained this thrust through the new millennium, as Peachey and Burton (2010) assert that transformational and transactional leadership has been the main focus in a variety of sporting contexts.

The popularity of transformational leadership studies in sport has provided managers and administrators seeking to improve their understanding of their organisation and of leadership abilities with practical advice and prescriptions. After thirty years of research in the area, Chelladurai (2011) presented a review of his work advocating vision creation, supportive leadership and personal recognition. Similarly, Slack and Parent's commentary 
(2006) on leadership in sport discusses theoretical applications to sports organisations and concludes the importance of and practicality of transformational leadership. Soucie (1994) has also drawn lessons from the leadership literature and provided advice for practicing sport administrators that align with many transformational dimensions.

Empirical research has also provided support for the conduct of transformational leadership in sport organisations. Within Canadian provincial sport organisations, Kent (1995) found that transformational behaviours might affect the organisational culture, which in turn, is positively linked to organisational effectiveness. Hsu and colleagues (2002) likewise argue that transformational leadership seems to be at least indirectly related to effective Taiwanese sport organisations through its effect on the culture. The direct effects of transformational leaders on organisational effectiveness have been evidenced in the administration of South African cricket (Ristow, Amos, \& Staude, 1999), along with follower job satisfaction in physical education organisations (Rizi et al., 2013).

Leadership in education has also evolved as a consequence of its changing environment. The public sector - and more specifically the educational sector - is expected to cope with a rapidly changing and turbulent environment through the adoption of private sector practices and leadership styles (Balyer, 2012; Parry \& Proctor-Thomson, 2003). In order to promote adaptability and change within a school, leaders may seek to adopt Bass's model, as "transformational leadership has similar effects on educational leaders such as school principals as it does on leaders in production and service organizations" (2000, p. 34).

The quality of school sport provision has a range of barriers, including financial constraints leading to reduced physical education (PE) budgets, lower importance of PE and sport, and inadequate resources both structurally and personnel-wise (Casper, Bocarro, Kanter, \& Floyd, 2011; Committee on Sports Medicine \& Committee on School Health, 1987; Marshall \& Hardman, 2000; Pope, 2011; Ross \& Cowley, 1995). More specifically, PE 
and school sport "is suffering from decreasing curriculum time allocation, budgetary controls with inadequate financial, material and personnel resources, has low subject status and esteem, and is being ever more marginalized and undervalued by authorities" (Marshall \& Hardman, 2000, p. 223). The apparent decline in the standing of PE and sport in schools was evident in the late 1980s (Marshall \& Hardman, 2000) and is still evident in New Zealand secondary schools more than thirty years later (Adamson \& Handcock, 2013).

In order to respond to the educational changes and overcome these barriers, Leithwood (1994) hypothesised that transformational leadership may be positively related to various school organisational conditions and outcomes. Leithwood began his research in the late 1980s and early 1990s, arguing that "transformational approaches to school leadership are especially appropriate to the challenges facing schools now and through the remainder of this decade" (1994, p. 499). According to Stewart's (2006) review of key authors for transformational leadership, Leithwood and his colleagues (Leithwood, 1992, 1994; Leithwood, Jantzi, \& Steinbach, 1999; Leithwood \& Jantzi, 1999, 2005) have been instrumental in bridging the work of Burns and Bass into the field of educational administration.

Prior studies have indicated that reform in secondary schools can be achieved through transformational leadership throughout various levels of management. Leithwood and Jantzi's most recent review (2005) provides a synthesis of 34 articles in elementary and secondary school outlining how transformational leaders may emerge, even in turbulent and resource constrained settings. School principals who demonstrate such behaviours have been shown to positively affect teacher job satisfaction, extra effort and commitment towards greater provision (Bayler, 2012; Geijel, Sleegers, Leithwood, \& Jantzi, 2003; McCarely, Peters, \& Decman, 2014; Sala, 2003). The positive outcomes can also be enhanced when 
decision-making is shared throughout the organisation (Copland, 2003) in positions such as senior management and sports coordinators.

Overall, the international literature relating to the sporting and educational sectors has explored the practicality and implications transformational leadership, concluding that it may be a pertinent and useful style for managers to adopt. More specifically, school sport managers are faced with challenges of operating in an unstable environment with scarce resources. Scholars suggest that overcoming these constraints may be possible through transformational leadership behaviours to enhance organisational effectiveness.

\section{New Zealand Studies}

Empirical research has been conducted in New Zealand exploring the prevalence and affects of transformational leadership theory on organisational outcomes such as job satisfaction, extra effort and perceived leader effectiveness. It is important to compare and contrast findings to other nations as the cultural values, beliefs and norms may determine the behaviours of leaders and in turn the implications these have on followers.

In congruence with Western countries such as the United States, transformational leaders appear to be highly valued and prevalent in New Zealand organisations (Bass \& Riggio, 2006). Originally Bass's (1985) book examined MLQ self-reports of 23 New Zealand school administrators, concluding significant effect sizes for transformational and contingent reward behaviours. Other researchers have applied transformational leadership theory to understanding leadership in New Zealand companies. Most notably, findings showed that followers and leaders alike prefer working with personnel who are more transformational in nature (Singer, 1985; Singer \& Singer, 1986; 1990).

In addition to subordinates preferring to work for leaders who exhibit transformational leadership, the New Zealand leaders also mirror the hierarchical of correlations among Full Range Model of Leadership styles and outcomes in effectiveness 
(Bass, 1997). The pattern emerged for New Zealand professionals and administrators (Bass, 1985; Singer, 1985) whereby the most effective behaviours were transformational, followed by contingent reward and the relatively ineffective behaviours of management-byexception and laissez-faire.

The three measures of effectiveness commonly explored through the MLQ have been job satisfaction, extra effort and perceived-leader effectiveness. Firstly, transformational leaders appear to satisfy subordinates to a greater extent than transactional leaders, regardless of national context. Research within New Zealand has provided similar results. Most notably, University of Canterbury's researchers, Ming and Alan Singer, explored the applicability of Bass's (1985) theory in New Zealand. Singer's (1985) study of 38 randomly selected New Zealand company managers found that transformational leadership factors were more highly correlated than transactional behaviours with job satisfaction. Building on these findings, Singer and Singer $(1986 ; 1990)$ surveyed the relationships between leadership preference and subordinate personality, revealing that followers will be more satisfied with transformational leaders.

In regards to the remaining organisational effectiveness measures, little is known within the New Zealand context. Support for the association between transformational leadership and perceived leader effectiveness has been found in empirical research within New Zealand companies (Singer, 1985; Singer \& Singer, 1986). However, a greater understanding for the implications leadership has on extra effort is needed as no significant associations have been found.

Although there is a lack of knowledge on the effects of leadership on organisational outcomes, there is an apparent utility of the transformational leadership theory within New Zealand. The relatively high prevalence and preference for transformational leaders may be due to the egalitarian, collectivistic and flat hierarchical structure of industries that have 
characterised New Zealand since the 1980s (Bass, 1985). Empowering and shared leadership has relevance in such a setting, as perhaps suggested by Parry and ProctorThompson (2003) and Bass and colleagues (1987), who found support for the falling dominoes effect in the New Zealand public sector.

Consequentially, transformational leadership appears to warrant further investigation in different New Zealand organisations. Previous academic literature has suggested that the 'New Leadership theory' has been the most favoured and relevant style for organisations across the country. Nevertheless, there is a gap in knowledge regarding how the transformational leadership style affects organisational effectiveness. It is important to understand the relationship in this context as it may determine the appropriate behaviours for current and future leaders.

\section{Sports Coordinators}

A school sports coordinator has become an established role within the New Zealand school sports system, whereby the role is often to formulate sporting plans and coordinate and oversee programmes (New Zealand Secondary School Sports Council, 2011). Sport NZ has outlined that those responsible for running secondary school sport must understand the maelstrom of requirements of all parties; these include educational curriculum and sporting needs of youth, management of volunteers and coaches, and collaborating with both internal and external entities to enhance financial, structural and personal capacities (Sport NZ, 2008, 2014).

The successful sports administrator will also be responsible for empowering and motivating subordinates to establish goals and seek their attainment (Soucie, 1994). Moreover, athletic directors or sports coordinators must articulate a vision for their department's future and possess the professional and technical skills to see their vision 
become a reality (Armstrong, 1992). Similar to North American athletic directors, Weese stressed their success can be determined by effective leadership practices,

"While it could be argued that effective leadership is required in any sport management setting, it may be particularly necessary in the campus recreation area. Directors of these programs are presented with perennial budget reduction and a smaller work force mandated to accomplish more with fewer resources" (1996, p. 199-200).

The leadership characteristics and posited benefits of transformational leadership in school sport programmes have predominantly been explored through doctoral dissertations in American intercollegiate athletics (Soucie, 1994). Some of the earliest pieces of research identified a paucity of transformational athletic directors and coaches (Armstrong, 1992), and by comparison, high frequencies of laissez-faire (Rouff, 2007). However, more contemporary studies have reported higher frequencies of transformational behaviours in athletic directors and coaches (Day, 2013; MacDonald, 2012) that may contribute to more effective and successful provision.

The effects that leadership styles have on organisational effectiveness have seen mixed results throughout a range of varying athletic departments. For instance, Kuchler (2001), Linam (1999) and Choi (2006) found the coaches' of American NCAA teams were not more satisfied in their roles when their athletic directors displayed transformational dimensions. On the other hand, positive correlations have been found more recently in selected NCAA schools (Rouff, 2007). Overall, findings suggest that a variety of factors may determine coaches' satisfaction in their roles and these may be out of the control of the leader.

Although past research has produced contradictory results for coaches' satisfaction, support for the utility of transformational leadership amongst athletic directors has been found in other dimensions of organisational effectiveness. For example, coaches' perceive their Directors' leadership to be more effective when displaying such behaviours (Choi, 2006; 
London, 1996; Miyashiro, 2007). More importantly, Macdonald's (2012) MLQ survey of ninety-six athletic directors concluded that transformational leadership might elicit greater effort from coaches.

The few numbers of scholarly articles that have been published in academic journals reveal general encouragement for athletic directors to exhibit transformational behaviours. Doherty and Danlychuk (1996) found that athletic leaders displayed behaviours across the whole Full Range of Leadership continuum, however, transformational behaviours were more highly correlated to coaches' satisfaction, extra effort and perceived leader effectiveness. A year later, Doherty (1997) revealed that mediating variables including gender, age and years of experience might determine leadership styles and resultant organisational outcomes. In spite of this, Kent and Chelladurai (2001) produced contrasting relationship, finding no associations between leadership and a number of organisational outcomes.

Attempting to generalise these findings to New Zealand secondary school sports coordinators may not be possible, given the different context and environments. According to a 2013 survey on employment conditions and characteristics, undertaken by the New Zealand Secondary Schools Sports Council, which 398 responses from sports coordinators, a number of interesting findings emerged. Most notably, the majority of sports coordinators only work on a part-time basis, and have less than three years' experience in the role. The lack of time available to part-time sports coordinators to interact with followers may make it difficult for the coordinators to act as a leader, or to encourage subordinates to act in line with the organisation's interests in mind.

Unlike the majority of NCAA schools who can fund the employment of skilled coaches, volunteers form the backbone of sport and recreation in New Zealand (SPARC, 2011). The need for volunteer coaches, managers and administrators is no different in secondary school sport as outlined by the Sport and Recreation Coordinators Manual (Sport 
NZ, 2008), and its guide to running secondary school sport (Sport NZ, 2014). According to Adamson and Handcock's (2013) survey of New Zealand sports coordinators, these volunteers are predominantly from within the school and include teachers, students and parents.

Despite the large proportion of volunteers who fill such roles, a common perception is that sports organisations still regard their volunteer base as being especially scarce (Wicker \& Breuer, 2011). Furthermore, the changing nature of modern sport has altered the volunteer experience, which in turn makes it more difficult to recruit and retain volunteers (Cuskelly, 2004; Cuskelly, Taylor, Hoye, \& Darcy, 2006). This can be attributed to the need for more 'business-like' practices (Gilbertson et al., 1999), the 'time-squeeze' phenomenon according to Sharpe (2006), different volunteer motivations (Kazakov \& Johnson, 2008), and barriers or dissatisfaction leading to quitting (SPARC, 2006).

As volunteers are not contractually obliged to continue with their work in sport organisations, these people who give up their valuable time, expertise, effort and skills can also easily leave an organisation at their own discretion, potentially harming the longevity of operations (Cuskelly, 2004). Correspondingly, Catano and colleagues (2001) claim leadership may play an important role in retaining personnel and improving the organisation's effectiveness. The transformational leadership model appears to be particularly applicable to volunteers through its ability to satisfy and inspire followers to exert extra effort and commitment.

A transformational leader in the organisation may be able to appease or support the intrinsic motivations for a range of volunteers. For instance, "volunteers are more satisfied with their service when team leaders are inspirational, show concern about their development, involve them in decisions, and focus on the meaning of work" (Dwyer et al., 2013, p. 143). Sport NZ's (formerly SPARC) document Finding and keeping volunteers outlines nine 
motivation segments of volunteers, which often can be met through transformational leaders (SPARC, 2006). For example, intellectual stimulation is what many Skill Seekers and Investors seek (SPARC, 2006). Such transformational leadership is also more effective than transactional when acknowledging contributions from followers (Bass \& Rigio, 2006). Extrinsic rewards and punishment are replaced with praise, which is actively sought by volunteers (i.e., The Frustrated). The personal nature of transformational leadership appears to offer practicality for sports coordinators as "Organisations need to develop a culture that reflects a greater 'customer care' philosophy and align their processes and practices more closely with what volunteers tell them is important to them" (SPARC, 2006, p. 6).

Empirical research from the early twenty-first century to today has found efficacy in transformational leadership behaviours in voluntary non-profit organisations. Within Australian rugby clubs and voluntary boards, leaders should be more relational than transactional in order to satisfy and retain volunteers (Cuskelly et al., 2006; Hoye, 2006). Transformational leadership which recognises the individual needs of volunteers may also create a more satisfied following that is willing to contribute more to their cause (Dwyer, Bono, Snyder, Nov, \& Berson, 2013; Parris \& Peachey, 2012; Rowold \& Rohman, 2009). Such findings suggest that transformational leadership behaviours may be useful for ensuring longevity of sport provision, particularly for those sport coordinators who lead a large volunteer-base.

In sum, New Zealand secondary school sports coordinators face an array of challenges in their pursuit of running successful sporting programmes. The literature suggests that leadership may provide them with an effective tool for overcoming their obstacles and ensuring organisational effectiveness. The benefits of transformational leadership have been illustrated in a range of settings including sport organisations (Soucie, 1994) and educational 
institutions (Leithwood \& Jantzi, 2005). Consequently, the appropriateness of leaderhsip style has been reinforced for New Zealand sporting managers by Gilbertson and colleagues stating:

"Given the very significant changes in our society and in the roles of sport and recreation... transformational leadership is the most relevant domain of study for leaders of New Zealand sport and recreation” (2000, p. 154).

In contrast to American sporting administrators, little is known about the leadership practices of New Zealand secondary school sport coordinators. The cultural contingencies that impact leadership practices within New Zealand may vary and result in significantly different organisational effectiveness outcomes to previously studied school sport leaders in the US (Doherty, 1996; Doherty \& Danylchuk, 1997). As "transformational leadership is an extremely popular image of ideal practice in schools at the present time" (Hallinger, 2003, p. 178), it is important to investigate the implications that sport coordinator leadership may have on organisational effectiveness in New Zealand.

Therefore, this research seeks to compare and contrast the leadership behaviours of New Zealand secondary school sport coordinators (i) amongst themselves and (ii) compared with the extant literature -and then whether and how consequential organisational outcomes impact on both internal and external stakeholders. 


\section{Chapter III: METHODOLOGY}

This chapter provides an outline of the processes and methodological procedures used to examine the leadership behaviours and organisational effectiveness of New Zealand secondary school sports coordinators in this Wellington region. Aspects of methodology discussed in this chapter include: (1) the research design; (2) the population surveyed; (3) the procedures followed; (4) the instruments and rationale for their inclusion; (5) a data analysis; and (6) the limitations of the methodological design.

\section{Research Design}

Aligned with research objectives intended to explore the applicability of the transformational leadership literature in sports coordinators, an interpretive paradigmatic stance has been taken for this study. Sparkes (1994) states that research studies from this perspective "argue that there are multiple realities and that the mind plays a central role, via its determining categories, in shaping and constructing these (multiple realities)" (p. 13). Implications for this study include a view that leadership behaviours and organisational outcomes can and will differ between sports coordinators and schools as meaning and experiences are socially constructed by and for various actors (Cavana Delahaye, \& Sekaran, 2001).

As the interpretive paradigm adopts an ontological assumption and acceptance of subjectivity, the validity and reliability often attributed to quantitative research findings are of less importance in this study, as it is not intended to generalize to all New Zealand secondary school sports coordinators. On the one hand, it is recognised that the approval or rejection of hypotheses applying statistical testing processes to data acquired using the MLQ instrument may offer insight on the statistical significance of different outcomes assumed to be related to 
the extant leadership styles of Wellington sports coordinators. On the other hand, it is also recognised that a major use of quantitative data is to provide a foundation for further enquiry and exploration into phenomena of interest. As such, for example, the validity and reliability of data measures relating quality are often measured through trustworthiness reflecting dependability and authenticity of findings (Cohen, Manion, \& Morrison, 2000; Golafshani, 2003).

For this study, it was decided to adopt a sequential explanatory design, with the purpose of supplementing quantitative data obtained from the MLQ instrument with semistructured interviews of sports coordinators. This sequential mixed-methods approach entails first collecting cross-sectional survey data, and then complementing the analysing of such quantitative data with the richer qualitative data obtained from individuals. Tashakkori \& Teddlie (2003) suggest that the use of such complementary data sources and complementary analysis would enable this study to more readily understand broader patterns and individualised behaviours arising from different school contexts. As such, the research design for this study has been chosen for similar reasons. For example, the 'quantitative' MLQ research instrument is used to explore leader demographics, leadership characteristics and their possible associations with measures of organisational effectiveness. Insights drawn from analysis of data can then be used to guide interview questions with individuals, thereby augmenting the quantitative data, and thereby seeking to provide more depth into patterns and trends.

This mixed-methods approach has "aims which are directed at providing an in-depth and interpreted understanding of the social world of research participants by learning about their social and material circumstances, their experiences, perspectives and histories" (Snape \& Spencer, 2003, p. 3). Its functions can be contextual, explanatory, evaluative, and generative according to Ritchie (2003). Combining the two methods not only allows a degree 
of testability, but also signals the importance of drawing context into the research, as two key aspects of the study.

Prominent authors who have reviewed leadership literature have also encouraged future research to adopt a mixed-methods approach. This point was evident even in the early developmental stages of transformational-transactional theory given the stated belief of Lowe and associates (1996) that a mono-methods bias may limit the generalizability of findings beyond the specific items measured through the MLQ. A sequential explanatory design may alleviate this problem and provide a context for, and enhanced understanding of findings from MLQ studies. A similar stance was taken in a more recent meta-analysis arguing for the implementation of a mixed-methods approach. Indeed, DeGroot et al. (2000, p. 364) claimed that a mixed-methods approach "must be employed to uncover the true relationship between variables." In addition, Leithwood and Jantzi's (2005) meta-analysis of transformational leadership studies in the school setting has shown that research has progressed from simply using the MLQ instrument for data collection, towards an acceptance of a qualitative and mixed-methods approach seeming to offer greater value.

For this study of leadership in the secondary school context, the limitations and deficiencies of both quantitative and qualitative research used alone, may be overcome through the mixed-methods approach (Creswell, 2002; Hanson, Creswell, Plano Clark, Petska, \& Creswell, 2005). Such limitations may relate to the narrow results generated from cross-sectional surveys which are also susceptible to a number of specific biases (Punch, 2003). By contrast, the subjective nature of interviews might also be vulnerable to reactive and instigator/investigator effects, and that personal responses may not be representative of the entire population (Maykut \& Morehouse, 2000; Patton, 2002). In addition, Yukl's study of managerial leadership and research has stated, "the limitations of each type of methodology make it imperative to use multiple methods in research on leadership (p. 278). Furthermore, 
as suggested by Fuller et al. (1996), by drawing on different, complementary data gathering methods it will be possible to provide greater understanding of organisational outcomes.

Thus, we note that data collected from the MLQ instrument is intended to explore the patterns of sport coordinators' leadership styles and behaviours whilst also determining the related impact that these styles have on coaches' job satisfaction, extra effort and perceived leadership effective. The semi-structured interviews then seek to explain why leadership is important for sport coordinators, how it may affect followers within the school sport setting, and when it is needed to achieve desired organisational outcomes.

\section{Participants}

The study has chosen to target those secondary school sports coordinators in the Wellington region as defined and listed in the Ministry of Education's (2014) directory and New Zealand Secondary School Sports Council database. The region includes Wellington City, Lower Hutt City, Upper Hutt City, Horowhenua District, Kapiti Coast District, Porirua City, Masterton District, and South Wairarapa District. The consequent sample frame of secondary schools comprised a total of forty-nine schools within the Wellington region,

These secondary schools vary according to their structure (private, public and integrated), gender (boys only, girls only and co-ed), location (urban or rural), decile and population. The second stage sample frame for the study consisted of the forty-nine participants who coordinate sport in a range of schools. Of the sample frame six schools were ruled out, as their sport coordinator was not available to be contacted. From the final sample twenty-six $(60 \%)$ sport coordinators responded to the survey questionnaire and were used in this study. At the next stage, four sports coordinators from a purposive convenience sample of schools with different characteristics agreed to participation in semi-structured interviews.

Convenience purposive sampling of individuals (Cavana et al., 2001) has been used, as it is believed necessary to gather information in depth on the specific behaviours and 
perceptions of sports coordinators deemed to be the most important individuals in secondary school sporting provision, and whose leadership behaviours may have most impact. Additionally, whilst face-to-face interviews with the whole set of sports coordinators was not feasible due to time constraints, there are also advantages accruing from researching a smaller set of sample individuals. In another context, not so relevant in this study, Cavana et al. (2001, p. 254) has suggested that "studying a sample rather than the entire population is also sometimes likely to lead to more reliable results, mostly because fatigue is reduced, resulting in fewer errors in collecting data, especially when the elements involved are large in number. In a few cases, it would also be impossible to use the entire population to learn about or test something”. The present study has chosen not to seek responses from athletes, parents, school staff, or coaches from lower-level teams given that they may not often be in a position to appropriately judge sports coordinator's leadership behaviours, thereby ensuring results are more accurate and reliable.

\section{Sampling Procedure and Participant Selection}

The names and contact details of the sports coordinators from Wellington secondary schools were not readily available from the Ministry of Education, New Zealand Secondary School Sports Council, or other governing organisations. This information was obtained by telephoning each school using contact details from the current schools directory (Ministry of Education, 2014). Sports coordinator information was then collated in a secure database used to directly telephone the potential participants to apprise them of the research study, and to seek their involvement. All of the contactable sports coordinators (forty-three) agreed to respond to the electronic survey questionnaire, whereas the remaining six schools either had their coordinator on leave, or were in the process of filling current vacancies in the position.

If a sports coordinator agreed to participate in the survey, they were sent an e-mail which included an information sheet outlining the purpose of the study (Appendix B); and a 
consent document to be completed by participants (Appendix A), and also a link to the online survey (Appendix E). The email was sent at midday on Monday the $16^{\text {th }}$ of February, as this had been identified as an ideal time and day, to gain an optimal response (Punch, 2003). A reminder phone call and email was sent to the sports coordinators a fortnight later, with the survey closing on the $2^{\text {nd }}$ of March.

Following the collection of survey data, the purposive convenience sample of potential interviewees were contacted via a personal telephone call to ascertain their availability for interview, and to provide further information. The direct contact with coordinators was also considered an important part of establishing an initial initial rapport, which can improve the ensuing interview process (Whiting, 2008). As the research objectives related to an exploration of leadership styles in a range of school settings, sport coordinators from different

school structures were desired. A second stage sample frame of 8 schools that were distinctly different in their structure, gender, location and student population were chosen and their coordinators were eager to be interviewed. However, due to time constraints and coordinator availability, only four sports coordinators could be participate.

Interviews were scheduled with the sports coordinators within the first three weeks of the 2015 school term. The recommended practices of Barriball \& White (1994) and Turner (2010) were followed, and semi-structured interviews of 30-60 minute duration, were conducted in private in the sports coordinators' offices. Questions followed the interview guide (Appendix D) and allowed probing of new questions from interesting responses.

\section{Instrumentation}

For the initial stage of data gathering, the MLQ instrument was used as a selfadministered online survey questionnaire. The procedure ensured anonymity, allowed sufficient time for response, and with the expectation that it could provide relevant data that was easily available at the least expense to the researcher (Dillman, 1991; Miller \& Salkind, 
2002; Mangione, 1995). An online survey allowed the research to gather data from a wide variety of geographical locations across the Wellington region, which would not have necessarily been available through personal contact. As stated above, it also allowed the respondents some privacy and time to collate relevant information, and consider their answers (Mangione, 1995).

In Chapter II, it was stated that Bass (1985) first developed the MLQ instrument with items measuring three dimensions of transformational leadership termed as: charismatic leadership, intellectual stimulation, and individualized consideration. Over time, a range of additions, alterations, and adaptations (Avolio, Bass, \& Jung, 1999; Bass, 1995, 1997; Bass \& Riggio, 2006) has been made resulting in a variety of MLQ instruments. For this study, the Multifactor Leadership Questionnaire Short-5X Self-Rater version was employed.

This version of the MLQ allows for operational measures of leadership styles and organisational effectiveness through a total of 45 questions, reflecting nine leadership dimensions, and three organisational dimensions (Avolio \& Bass, 2004). More specifically, the nine-factor Full Range Model of Leadership allows for the identification of five dimensions of transformational leadership: Idealised Influence (Attributed), Idealised Influence (Behaviour), Inspirational Motivation, Intellectual Stimulation, and Individualised Consideration; three dimensions of transactional leadership: Contingent Reward, Management-by-Exception (Active), and Management-by-Exception (Passive); whilst a 'nonleadership' dimension is operationally defined and identified as as Laissez-Faire. Moreover, organisational outcomes of perceived leader effectiveness, subordinate job satisfaction and extra effort are quantified through nine questions. Examples of instrument items can be found in Appendix E, for instance, question thirty-four constitutes an Idealised Influence (Behaviour) style:

'I emphasize the importance of having a collective sense of mission' 
Table 1 presents the leadership and organisational outcome subscales as well as the questions, which were summed up to represent these dimensions.

TABLE 1.

Scoring of MLQ 5X-Short Self-Rater Instrument

\begin{tabular}{|l|l|}
\hline \multicolumn{1}{|c|}{ Scale } & \multicolumn{1}{c|}{$\begin{array}{c}\text { Instrument } \\
\text { Items }\end{array}$} \\
\hline Idealised influence (Attributed) & $10,18,21,25$ \\
\hline Idealised Influence (Behaviour) & $6,14,23,34$ \\
\hline Inspirational Motivation & $9,13,26,36$ \\
\hline Intellectual Stimulation & $2,8,30,32$ \\
\hline Individualised Consideration & $15,19,29,31$ \\
\hline Contingent Reward & $1,11,16,35$ \\
\hline Management-by-Exception (Active) & $4,22,24,27$ \\
\hline Management-by-Exception (Passive) & $3,12,17,20$ \\
\hline Laissez-Faire & $5,7,28,33$ \\
\hline Extra Effort & $39,42,44$ \\
\hline Perceived Leader Effectiveness & $40,43,45$ \\
\hline Job Satisfaction & 38,41 \\
\hline
\end{tabular}

Respondents were asked to assess how frequently/how often each instrument item/statement reflected activities undertaken in their role as school sport coordinator, and to circle the point that best resembled their judgement. The instrument items are structured around 5-point interval scales ranging from 'Not at all' to 'Frequently, if not always'. Such an interval scale "allows [the researcher] to perform certain arithmetical operations on the data collected from the respondents" (Cavana, Delahaye, \& Sekaran, 2001, p. 196). Consequentially, the magnitude of the differences in leadership behaviours can find an 
arithmetic mean along with the standard deviation, variance, and coefficient of variation (Cavana et al., 2001).

The MLQ instrument provides the most commonly used operational measures of transformational and transactional leadership, with the 'short' form (as used in this study) offering a reliable and valid measurement tool (Avolio et al., 1999; Avolio \& Bass, 2004). It may also yield a greater response rate when compared to the longer versions (Avolio \& Bass, 2003), which may increase the validity of findings. The validity of the measurement model of this short form of MLQ instrument has been explored by several researchers revealing a range of benefits across various contexts along with a stable factor structure, when subject to multivariate statistical factorial analysis (Antonakis et al., 2003; Meunjohn \& Armstrong, 2008; Rowold, 2006; Tepper \& Percy, 1994). Further support of the short form model's internal consistency has been found through statistical hypothesis testing using Cronbach alpha coefficient statistics relating to the overall leadership mean scores. Examination of interval scale reliability for the operational measures of different dimensions has found statistical reliability to be high (Den Hartog et al., 1997; Avolio et al., 1999), with statistical tests exceeding the standard cut-offs for internal consistency $(.70)$ recommended elsewhere in literature (Bass \& Avolio, 1997).

Supporting the use of the MLQ instrument in the context of this study, we draw on Bass \& Avolio's (1997) comment that "sport management researchers interested in quantitatively measuring transformational leadership would be well served in utilizing the valid and reliable Multifactor Leadership Questionnaire (MLQ)” (p. 188). In addition to comments made above, the instrument's utility and reliability is also evident in empirical articles on leadership within sport organisations (Doherty, 1997; Hsu et al., 2002; Kent \& Chelladurai, 2001; Ristow et al., 1999). In particular, Doherty and Danylchuk's (1996) study 
of athletic directors revealed statistically significant reliability coefficients (between .74 and .88).

The subsequent stage, providing a qualitative aspect of the study, follows a semistructured interview (see interview guide, Appendix D) that covers four main topics: participant/school information, leadership behaviours in sport coordinators, the full range model of leadership, and organisational outcomes. Questions were designed to be open-ended, and as neutral as possible, whilst also allowing new themes to emerge (Turner, 2010; Whitting, 2008). For example, "Can you tell me ... about what you think makes a good sports coordinator?" permitted probing and unearthed many of the challenges faced in this role.

An initial version of the interview guide and its questions was piloted in December 2014 with a range of relevant sources including current sport coordinators and teachers. This was carried out to identify any complex and confusing questions; to seek feedback for additional questions and factors that should be considered; and to test data collection methods (Gratton \& Jones, 2004). Following sound feedback, the semi-structured guide and questions were finalised for the qualitative data collection in February 2015.

\section{Quantitative Data Analysis}

In order to analyse the data collected from the questionnaires, the researcher engaged in analysis of selected statistical measures for each of the hypotheses. The statistical software analysis package, SPSS, was chosen as the vehicle for data analysis. The SPSS software analysis package is a widely used in the social sciences and business management, and enable quick and reliable statistical analysis through a range of measures, while also reducing human errors (Coakes \& Steed, 2009). As a general statement of the approach to hypothesis testing, for this study, the chance explanations offered in support of a null-hypothesis will be rejected if those explanations rely on chance occurrences falling below a 5\% probability level (ie if the 
p-value is less than 0.05). As such, a null-hypothesis will be rejected at a 5\% significance level, in favour of a previously stated alternative hypothesis..

However, prior to any analysis, data will initially be verified for accuracy and completeness. The data will then be subject to descriptive statistical analysis for key variables, the mean and standard deviations for each of the leadership behavioural variables as well as specified dependent variables. This will enable the researcher to gauge the frequency and intensity of perceived leader behaviours, as well as measures of organisational effectiveness identified as the dependent variables. Once these are determined it may be possible to categorise leadership behaviours and organisational effectiveness variables as high and low frequency relative to the mean. This will be used to illustrate patterns and relationships whilst also serving as groupings for statistical testing of observed differences and correlations.

Secondly, multivariate analysis, for example, Pearson correlation matrix analysis will be used to establish the existence of statistical relationships between and across variables. Statistical testing of Pearson correlation measures would allow the researcher "to see the nature, direction and significance at the bivariate relationships of the variables used... A Pearson correlation matrix will provide this information" (Cavana et al., 2001, p. 419). Furthermore, SPSS software will be used to conduct Chi-squared categorical analysis (Plackett, 1983) and Fisher's tests on each of the dependent variables, regrouped as, say, 'high/low' categories, to investigate their relationship with organisational effectiveness. Categorical groupings based on 'high' and 'low' scores relative to respective means, will allow the construction of $2 \times 2$ contingency tables to facilitate such tests. Chi-squared and Fisher's statistics may be used to complement and possibly further substantiate any general association suggested by the Pearson correlation measures. In addition, the Pearson 
correlation measures may provide a 'goodness of fit' for relationships that are specified as mathematical relationships.

\section{Qualitative Data Analysis}

An inductive approach to analysing the open-ended responses was selected, as it is a commonly used approach for interpretive studies (Gratton \& Jones, 2004). This was used to analyse each response in order to code information and find higher order themes. The basis of an inductive approach is that allows inductive coding and categorisation of raw data into conceptual categories that are perceived to be mutually exclusive and exhaustive (Gratton \& Jones, 2004). This analysis is of value, in as much as it is not predicated on categorisation according to pre-conceived or imposed themes, but rather that it allows themes or patterns to emerge through the researchers' interactions with the data (Patton, 2002). 


\section{Chapter IV: RESULTS}

In this chapter the analysis of both the quantitative and qualitative data retrieved from the survey and interviews are presented. The chapter is organised in line with the first three research questions, therefore it presents and discusses quantitative data first, followed by qualitative results. There will be interplay between the two, as some qualitative answers are used for explanatory purposes, enabling a greater depth of analysis of the sports coordinators' leadership behaviours. It must be noted that while respondents are expected to be answering some questions regarding sporting provision on behalf of their school, personal opinion may influence some individual responses.

The survey for this study was chosen to be the self-rater version of the MultiFactor Leadership Questionnaire (MLQ). The survey was electronically sent to forty-three willing Sports Coordinators, with a total of 26 completed responses $(60 \%)$. The response rate was considered satisfactory, as research has shown that, on average, around $40 \%$ of surveys sent by email will be returned (Super Survey, 2009).

The reliability for each subsection of the survey instrument has been supported previously through rigorous testing (Den Hartog et al., 1997; Avolio et al., 1999), concluding each of the constructs of leadership and organisational outcomes are capable of accurately measuring the correct dimensions (Bass \& Avolio, 1997). Cronbach Alpha tests were performed through SPSS software for transformational, transactional and laissez-faire leadership scales. Additionally, the nine factors that comprise the above leadership styles and the three organisational outcomes (extra effort, perceived leader effectiveness and job satisfaction) were tested to examine if the survey questions accurately measure the scales according to sport coordinator's responses. 
Cronbach Alphas for the MLQ ranged from .58 to .87 (with two exceptions) as shown in Table 2, indicating that most subscales meet sufficient reliability estimates.

TABLE 2

Cronbach's Alpha scores for MLQ sub-scales

\begin{tabular}{ll}
\hline MLQ Sub-Scales & Cronbach's Alpha \\
\hline Transformational Leadership & .87 \\
Idealised Influence (Attributed) & .62 \\
Idealised Influence (Behaviour) & .70 \\
Inspirational Motivation & .75 \\
Intellectual Stimulation & .69 \\
Individualised Consideration & .68 \\
Transactional Leadership & .57 \\
Contingent Reward & .62 \\
Management-by-Exception (Active) & .58 \\
Management-by-Exception (Passive) & .26 \\
Laissez-Faire & .70 \\
Organisational Outcomes & .26 \\
Job Satisfaction & .77 \\
Extra Effort & \\
Perceived Leader Effectiveness & \\
\hline
\end{tabular}

Most notably, Cronbach's Alphas for transformational leadership were good at .87; the style's five dimensions scores on average were .69, which is relatively acceptable. Alphas of .70 for laissez-faire, .74 for job satisfaction, and .77 for perceived leader effectiveness were also satisfactory. Two significant scales were deemed poor due to low correlations in their items, pertaining to the constructs of transactional leadership (.57) and extra effort (.26). 
The questionable transactional alpha scores may have been a consequence of the relatively small sample size $(\mathrm{N}=26)$ according to Coakes and Steed (2009). Furthermore, many scholars proclaim that passive management-by-exception may in fact be a nonleadership dimension (Bass, 2000) thereby lowering the reliability of transactional question items. Lastly, if the item 'I get others to do more than they expected to do' were to be removed from the extra effort construct, reliability would significantly rise to .80 .

Although this application of the MLQ produced debatable internal reliability scores for a select few items, these were not altered or removed due to the nature of this research. The objectives were to explore a previously unknown context to determine the applicability of past theoretical models and findings, not to ascertain cause and effect of leadership behaviours.

In addition to the survey, four sports coordinators were willing to participate in semistructured interviews. These interviews ranged from 30-minutes to over an hour in length. Questions (Appendix D) were aimed at uncovering a deeper understanding of the background of the coordinators; the structure and roles within the position; the current status of secondary school sport; leadership profiles of coordinators and how these relate to organisational outcomes. Previous research has shown discrepancies in leadership behaviours according to personal characteristics (i.e. gender, age, ethnicity) (Avolio, 1999; Doherty, 1997) along with the organisational structures and systems (Lowe et al., 1996; Judge \& Piccolo, 2004). Interviewees covered a range of demographics and school structures, which was important for validity of findings. There was an even split with gender (two females and two males) and the age of participants ranged from 25 years-of-age to 55 years-of-age. All participants came from various backgrounds and their experience in the role also varied. 


\section{Research Question 1}

The first research question aimed to determine the leadership profiles of Wellington Secondary School Sports Coordinators. Moreover, these profiles will enable an analysis of the degree to which they exhibit transformational leadership behaviours. Sports coordinators were asked to describe and evaluate their leadership behaviours based the 45 items of the self-rater version of the MLQ (see Appendix E). The items are constructed to reflect the five transformational leadership behaviours (idealised influence (attributes), idealised influence (behaviours), inspirational motivation, intellectual stimulation and individualised consideration), three transactional leadership behaviours (contingent reward, active management-by-exception and passive management-by-exception), and finally one factor for laissez-faire, or non-leadership.

Participants were asked to answer each question using a 5-point Likert Scale ranging from 0 (Not at all), to 4 (Frequently, if not always) to determine how often they display the particular behaviour. For example, "I consider each individual as having different needs, abilities, and aspirations". Each item represents either one of the constructs from the ninefactor model of leadership or one of the three organisational outcomes. As Mind Garden does not allow the MLQ questions to be fully displayed in doctoral dissertations the items have been coded with a key word so as to reflect their nature. In addition, the following page provides the mean scores and standard deviations from each question measuring the sport coordinator's leadership profile. 
TABLE 3

Wellington Secondary School Sports Coordinator Responses to the MLQ leadership items.

\begin{tabular}{|c|c|c|c|c|c|}
\hline & $\begin{array}{l}\text { Item } \\
\text { number }\end{array}$ & Mean & & $\begin{array}{l}\text { Item } \\
\text { number }\end{array}$ & Mean \\
\hline $\begin{array}{l}\text { Idealised Influence } \\
\text { (Attributed) }\end{array}$ & & & Contingent Reward & & \\
\hline Pride & 10 & 2.80 & Assistance & 1 & 2.64 \\
\hline Self-interest & 18 & 3.46 & Responsibility & 11 & 2.54 \\
\hline Respect & 21 & 3.57 & Goals & 16 & 2.96 \\
\hline Confidence & 25 & 2.64 & Satisfaction & 35 & 3.27 \\
\hline $\begin{array}{l}\text { Idealised Influence } \\
\text { (Behaviour) }\end{array}$ & & & $\begin{array}{l}\text { Management-by-Exception } \\
\text { (Active) }\end{array}$ & & \\
\hline Values & 6 & 2.36 & Attention & 4 & 1.96 \\
\hline Purpose & 14 & 3.42 & Problems & 22 & 1.92 \\
\hline Ethical & 23 & 3.35 & Mistakes & 24 & 2.08 \\
\hline Mission & 34 & 2.69 & Failures & 27 & 1.17 \\
\hline Inspirational Motivation & & & $\begin{array}{l}\text { Management-by-Exception } \\
\text { (Passive) }\end{array}$ & & \\
\hline Optimistic & 9 & 3.08 & Intervene & 3 & 1.08 \\
\hline Enthusiasm & 13 & 3.42 & Wait & 12 & 0.35 \\
\hline Vision & 26 & 2.40 & Broke & 17 & 1.96 \\
\hline Achievement & 36 & 3.27 & Chronic & 20 & 0.28 \\
\hline Intellectual Stimulation & & & Laissez-Faire & & \\
\hline Assumptions & 2 & 2.68 & Avoidance & 5 & 0.38 \\
\hline Seek & 8 & 3.12 & Absent & 7 & 0.19 \\
\hline Perspectives & 30 & 2.69 & Avoid decision & 28 & 0.62 \\
\hline Suggest & 32 & 2.88 & Delay & 33 & 0.65 \\
\hline \multicolumn{6}{|l|}{$\begin{array}{l}\text { Individualised } \\
\text { Consideration }\end{array}$} \\
\hline Coaching & 15 & 2.92 & & & \\
\hline Individuals & 19 & 3.38 & & & \\
\hline Needs & 29 & 3.42 & & & \\
\hline Help & 31 & 3.16 & & & \\
\hline
\end{tabular}


The responses from above table were then collated into each of the nine leadership factors. Table 4 displays the descriptive data of the summarised leadership profiles of sport coordinators in the Wellington region, according to the MLQ 5X-Short Self-Rater version.

\section{TABLE 4}

Wellington Secondary School Sports Coordinator Leadership Sub-scales

\begin{tabular}{llll}
\hline Leadership Sub-scales & Mean & Std Dev. & Std. Error \\
\hline Transformational Leadership & 3.01 & 0.57 & 0.11 \\
Idealised Influence (Attributes) & 3.14 & 0.66 & 0.13 \\
Idealised Influence (Behaviours) & 2.86 & 0.81 & 0.16 \\
Inspirational Motivation & 3.08 & 0.63 & 0.12 \\
Intellectual Stimulation & 2.85 & 0.59 & 0.11 \\
Individualised Consideration & 3.23 & 0.60 & 0.15 \\
Transactional Leadership & 1.86 & 0.51 & 0.10 \\
Contingent Reward & 2.87 & 0.74 & 0.15 \\
Active Management-by-exception & 1.80 & 0.79 & 0.16 \\
Passive Management-by-exception & 0.90 & 0.52 & 0.10 \\
Laissez-Faire Leadership & 0.47 & 0.66 & 0.13 \\
\hline
\end{tabular}

Results indicate that sports coordinators display distinctly more transformational leadership $(M=3.01, S D=0.57)$ behaviours than transactional $(M=1.86, S D=0.51)$ or laissez-faire leadership $(M=0.47, S D=0.66)$. From this data it can be concluded that the sports coordinators most frequently exhibit individualised consideration $(M=3.23, S D=$ $0.60)$, followed by idealised influence (attributed) $(M=3.14, S D=0.66)$ and inspirational motivation $(M=3.08, S D=0.63)$ in their relationships with coaches. 
A similar pattern in the frequency of behaviours emerged supporting Bass's (1985) proposed hierarchy. The transformational-transactional continuum is illustrated below in Figure 1 clearly displaying a drop-off in frequency of behaviours when nearing the nonleadership end. It is also apparent that transformational leader behaviours and contingent reward were utilised by sports coordinators 'Fairly Often' as reinforced by Table 4 listed all six constructs with means above 2.85 . The dip in frequencies visibly starts with active management-by-exception only occurring 'Sometimes' to 'Once in a While' $(M=1.80, S D=$ $0.79)$, trending downwards to passive $(M=0.90, S D=0.52)$, and reaching the least observed leadership behaviour of laissez-faire $(M=0.47, S D=0.66)$ occurring 'Once in a While' to 'Not at all'.

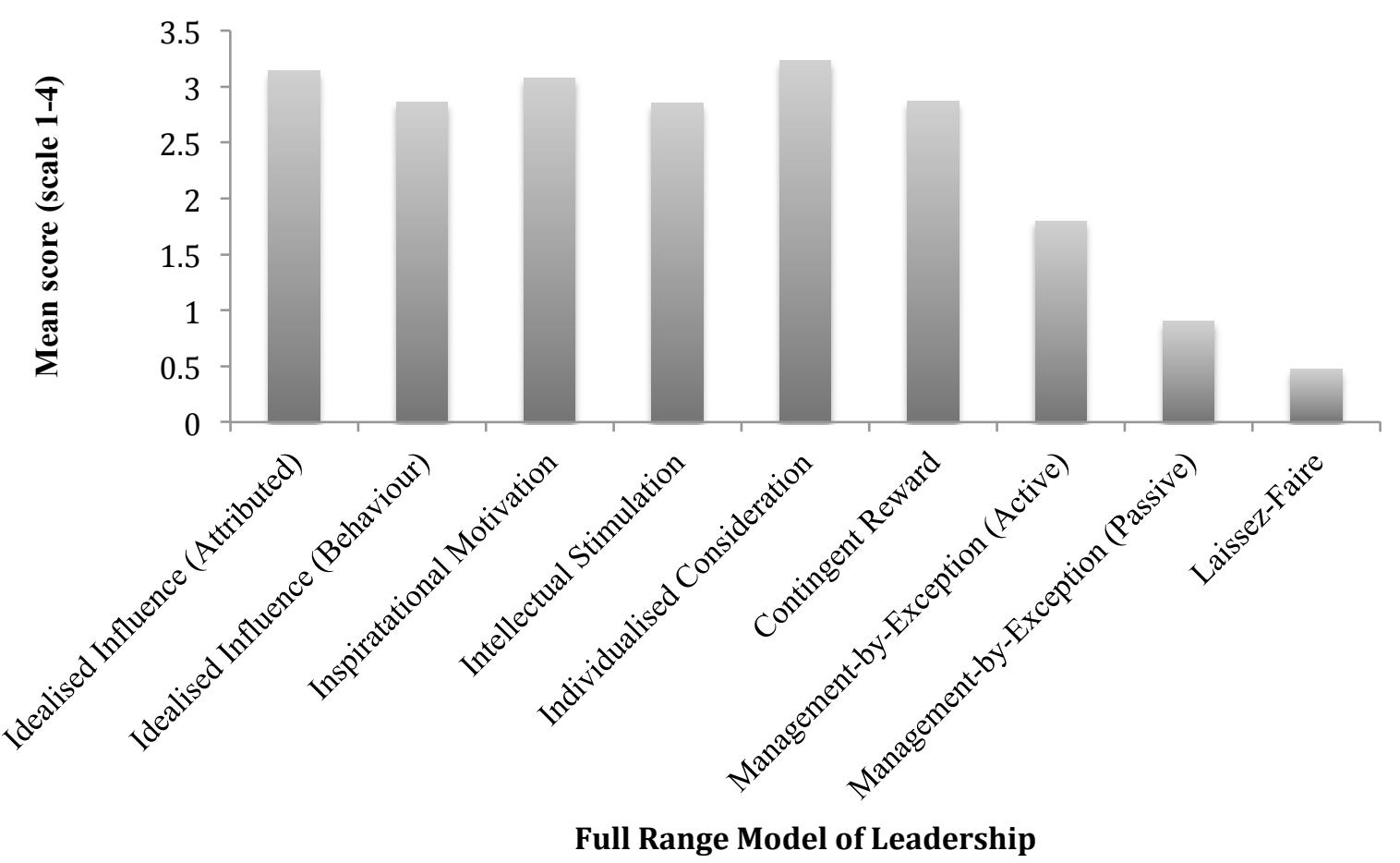

\section{FIGURE 1}

Frequency of mean leadership behaviours exhibited by Wellington Secondary School Sport Coordinators. 
The frequency of sports coordinators exhibiting certain leadership behaviours was also found not to be independent of one another. The correlation matrix (see Table 4) indicates the significant correlations among several leadership dimensions. Pearson Correlation tests performed on the data through SPSS software found a number of important associations between leadership behaviours. First, sport coordinators who display any of the transformational leadership behaviours will often simultaneously display the other four dimensions. All five constructs except idealised influence (attributed) and individualised consideration $(r=0.23)$ were significantly correlated with one another at a p-level of 0.05 or higher. For instance, inspirational motivation held an extremely positive association $(\mathrm{p}<.001)$ with idealised influence (behaviour) $(r=.79)$, intellectual stimulation $(r=.74)$, individualised consideration $(r=.70)$ and a very significant correlation with idealised influence (attributed) $(r=.52, p<.01)$. Interestingly contingent reward was positively correlated to all transformational behaviours bar idealised influence (attributed), providing potential support for the augmentation effect (Bass, 1985; Yammarino \& Bass, 1990). Finally, laissez-faire was negatively associated with idealised influence (attributed), inspirational motivation and individualised consideration ( $r=-.01, r=-.04$, and $r=-.05$ ), although these correlations were not significant. 
TABLE 4

Pearson's Correlations matrix for leadership behaviours and organisational outcomes.

\begin{tabular}{llllllllll}
\hline & \multicolumn{1}{c}{$A T T$} & BHV & MTV & STM & CSD & CTG & ACT & PAS & LF \\
\hline ATT & 1.00 & & & & & & & & \\
BHV & $0.48^{*}$ & 1.00 & & & & & & & \\
MTV & $0.52^{* *}$ & $0.79^{* * *}$ & 1.00 & & & & & & \\
STM & $0.44^{*}$ & $0.69^{* * *}$ & $0.74^{* * *}$ & 1.00 & & & & & \\
CSD & 0.23 & $0.53^{* *}$ & $0.70^{* * *}$ & $0.65^{* * *}$ & 1.00 & & & & \\
CTG & 0.36 & $0.73^{* * *}$ & $0.67^{* * *}$ & $0.80^{* * *}$ & $0.54^{* *}$ & 1.00 & & & \\
ACT & 0.17 & 0.34 & 0.27 & 0.35 & -0.01 & 0.31 & 1.00 & & \\
PAS & 0.12 & $0.39^{*}$ & 0.19 & 0.12 & -0.13 & 0.11 & $0.54^{* *}$ & 1.00 & \\
LF & -0.01 & 0.16 & -0.04 & 0.23 & -0.05 & 0.22 & 0.27 & 0.39 & 1.00 \\
\hline
\end{tabular}

Notes: ${ }^{*} \mathrm{p}<0.05 ;{ }^{*} \mathrm{p}<0.01 ;{ }^{* * *} \mathrm{p}<0.001$; ATT $=$ idealised influence (attributes) scale; BHV $=$ idealised influence (behaviours) scale; MTV = inspirational motivation scale; STM = intellectual stimulation scale; $\mathrm{CSD}=$ individualised consideration scale; $\mathrm{CTG}=$ contingent reward scale; ACT = management-by-exception (active) scale; PAS = management-byexception (passive) scale; $\mathrm{LF}=$ laissez-faire scale.

Sports coordinators who engaged in interviews provided similar responses. The participants believed that "all three styles are covered within the spectrum of school sport" (Sport coordinator 2). Of these styles, interviewees stated that both transactional and transformational styles are needed and more commonly used; coordinators need to be display a range of behaviours according to the context. Sport coordinator 1 explained, "You can't be one person and get along with everyone. You've got to be able to change your style and approach people differently". Qualitative responses may explain the strong correlations between leadership constructs found in the MLQ data.

When questioned on their own leadership behaviours sports coordinators claimed they exhibited transformational behaviours most frequently. All four interviewees believed their leadership style includes at least one aspect related to the transformational construct. Sport coordinator 1 was relatively new to the role and believed individualised consideration to be 
integral to how they established relationships in the role. Relationship maintenance was crucial when seeking to establish programmes and monitor progress.

The coordinators also all spoke of their application of idealised influence and inspirational motivation. A common theme within the leadership profiles was their desire to change attitudes of followers to move beyond their own interests and contribute towards attainment of the sport department's vision. Sports coordinator 4 spoke of how their influence diffused through the school creating a culture of shared purpose, "It's not about you're going to get a reward at the end, it's more about being a part of something". Similarly, sports coordinator 3 claimed,

SC3: "You need less self-interest. Kids need to be aware of the fact that sport is only ever functional when people are giving up their own time and expertise. So, it's an attitude change and awareness".

Sport coordinator 1 outlined how they exerted inspirational motivation to inspire followers to "common goals to make sport bigger and better in the school and help all the coaches and managers through administration and finance". Moreover, they provided a recent example of their leadership in action within the secondary school board,

SCI: “There's inspirational motivation where we're putting together a three-year plan and goals we want to achieve. The board is a hard thing to come up against but I think we got across our vision quite well and I think with some of the examples that we had in making this school a really big sports school and you know the funds that would come in from it were a big thing. So I think we inspired them a bit!"

Transactional behaviours, according to Sport coordinator 2 and 3, were also inherent in their leadership style. This occurred more for the management responsibilities of the role and for monitoring and rewarding coaches - when possible. 
Finally, no sports coordinator talked of passive or laissez-faire styles in their own leadership behaviours. Those who 'do not display any leadership behaviours or traits; rather, they avoid accepting their responsibilities, are absent when needed, fail to follow up requests for assistance, and resist expressing their views on important issues' (Appendix E) would be a rarity in the role. "They wouldn't last long if they didn't follow up with requests for assistance" (Sports coordinator 1).

\section{Research Question 2}

The second research question pursued an indicative and illustrative understanding of how leadership behaviours may affect organisational outcomes. More specifically, the research asked:

Are there any significant relationships between sport coordinators self-rated leadership styles (transformational, transactional and laissez-faire) and organisational effectiveness measures (coaches' job satisfaction, extra effort and leader effectiveness)?

The mean scores, standard deviations and standard errors of the mean were initially calculated through Mind Garden's electronic survey software and are presented in Table 5. Sports coordinators were asked to rate their influence on coaches' job satisfaction through questions 38 and 41 of the MLQ. Overall, coordinators perceived their methods of leadership and manners in which they work with coaches to be very satisfactory and occur frequently $(M$ $=3.33, S D=0.88)$. Perceived leader effectiveness was also rated very highly $(M=3.38, S D=$ 0.52) and measured through questions $37,40,43$, and 45. Lastly, sports coordinators viewed their leadership abilities to only 'Sometimes' influence coaches to apply extra effort according to a mean score of 2.58. Most notably, question 39 "I get others to do more than they expected to do" $(M=1.74, S D=0.73)$ only occurred 'Once in a while' to 'Sometimes'. 


\section{TABLE 5}

Organisational Effectiveness Measures as rated by Sports Coordinators

\begin{tabular}{llll}
\hline $\begin{array}{l}\text { Organisational } \\
\text { Outcome }\end{array}$ & Mean & Standard Deviation & $\begin{array}{l}\text { Standard Error of } \\
\text { the Mean }\end{array}$ \\
\hline Job Satisfaction & 3.33 & 0.88 & 0.17 \\
Extra Effort & 2.58 & 0.73 & 0.14 \\
$\begin{array}{l}\text { Perceived Leader } \\
\text { Effectiveness }\end{array}$ & 3.38 & 0.52 & 0.10 \\
\hline
\end{tabular}

Congruence in results was also uncovered from interviews when sports coordinators were asked to quantify how their leadership affects organisational outcomes on a scale of one to five. When questioned 'how satisfied do you believe your followers are with your leadership' the four coordinators rated their styles as highly satisfactory. These rating were manifested in the quote,

SC2: "I think if you value your staff they always want to try and do the best for you; if you act like you don't care or you're not interested then [unsatisfied coaches question] 'ohh what the hell am I doing this for?!' So definitely a four and a half'

In regards to their perceived effectiveness, most consider themselves to be successful in their roles. Sport coordinator 3 gave a rating of five (highly effective) and believed perceptions of their performance were pleasing from a number of stakeholders, such as the students, principal and teachers. Contrasting this, sport coordinator 1 rated their effectiveness to be "right around the middle, just yet as I haven't had a chance to really exert my leadership because everything I'm doing at the moment is I'm still learning and it's all still new". This may suggest that effectiveness is mediated by experience and tenure in the role.

Finally, interviewees didn't believe they could influence extra effort to the same extent as the previous two outcomes. Ratings were 'probably middle of the range' (Sport coordinator 2) as "we can motivate to a certain point to influence people but I don't know" 
(Sport coordinator 3). Although sport coordinator 1 believed "extra effort goes a long way", the other three coordinators were more reserved in assuming their abilities to influence greater effort.

SC2: "Teachers are teachers at the end of the day and if they want to help they will. A lot help for the right reasons - they want to help the boys and be able to help for whatever reasons [to] manage and coach or whatever. But at the same time there's a lot of people that don't want to be there and I think they would be there whether they wanted to be there or not"

This quote suggests that many teachers may not volunteer for roles as a response to the influence of sports coordinators; rather, they may contribute due to school expectations and job requirements. Sport coordinator 4 summarised this point,

SC4: "I think when they do it, they do it and I think they are prepared to do it for the same reasons. It's not because I asked them. There's a requirement and they're quite happy to do it".

Following the analysis of descriptive statistics, correlational tests were performed on the quantitative data provided by the MLQ self-rater $5 X$ form to test a number of hypotheses. Questions were framed around null hypotheses for each leadership construct in the nine-factor model and their associations with coaches' extra effort, job satisfaction, and perceived leader effectiveness. The SPSS software programme was used to analyse the data through two statistical measures; Pearson correlation tests were performed to illustrate any significant correlations between mean scores whereas the chi-squared and Fisher's tests determined how likely it is that the observed distribution between high and low scores is due to chance.

Findings will be presented in line with the Full Range Model of Leadership continuum, meaning transformational leadership and its five subscales will be explored first, 
followed by transactional leadership and its three subscales, and lastly the laissez-faire style. A summary of the Pearson correlation results is presented in Table 6 below, followed by results from each hypothesis.

\section{TABLE 6}

Matrix correlation between sport coordinators' self-rated leadership styles and organisational outcomes

\begin{tabular}{|c|c|c|c|}
\hline & Job Satisfaction & Extra Effort & Effectiveness \\
\hline$M(S D)$ & $3.33(0.88)$ & $2.58(0.73)$ & $3.38(0.52)$ \\
\hline Transformational Leadership & $0.42 *$ & $0.43 *$ & $0.49 *$ \\
\hline Idealised Influence (Attributes) & $0.48^{*}$ & $0.51 *$ & $0.62 * *$ \\
\hline Idealised Influence (Behaviours) & 0.32 & 0.32 & $0.40 *$ \\
\hline Inspirational Motivation & $0.41 *$ & $0.44^{*}$ & $0.56^{* *}$ \\
\hline Intellectual Stimulation & 0.31 & $0.44^{*}$ & 0.34 \\
\hline Individualised Consideration & 0.36 & 0.15 & 0.29 \\
\hline Transactional Leadership & 0.33 & $0.53 * *$ & 0.37 \\
\hline Contingent Reward & 0.31 & $0.63^{* *}$ & $0.42 *$ \\
\hline Active Management-by-exception & 0.12 & 0.26 & 0.29 \\
\hline Passive Management-by-exception & 0.33 & 0.23 & 0.04 \\
\hline Laissez-faire Leadership & 0.06 & 0.34 & -.005 \\
\hline
\end{tabular}

Notes: ${ }^{*} \mathrm{p}<0.05 ; * * \mathrm{p}<0.01 ; * * * \mathrm{p}<0.001$ 


\section{Transformational Leadership Behaviours}

\section{Null Hypothesis One}

There is no significant relationship between sports coordinators who exhibit transformational leadership and coaches' extra effort.

For this hypothesis, three tests were performed from SPSS software to determine the possible nature and direction of the relationship. The independent variable was measured through the means of the 5 I's provided by Mind Garden's data output; the dependent variable (extra effort) was tested through survey questions 39, 42, and 44 on the MLQ. The items only had 25 respondents; therefore, participant 1 was removed from data analysing effort.

A two-tailed Pearson correlation test was initially conducted, revealing a significant correlation between the two variables $(r=.43, N=25, p<0.05)$. Contrary to the initial findings, results from the chi-squared $(r=0.96, p=0.33)$, chi-squared with Yate's correction $(r=0.33, p=0.57)$, and Fisher's exact tests $(p=0.43)$ did not reveal any significant relationship between transformational leadership and extra effort. Although findings indicate that there may be a significant relationship in the population of Wellington secondary school sport coordinators, it appears that high levels of transformational leadership may not lead coaches to exert any extra effort. Thus, $\mathrm{H}_{\mathrm{o}} 1$ was only partially rejected.

\section{Null Hypothesis Two}

There is no significant relationship between sports coordinators who exhibit transformational leadership and coaches' job satisfaction.

For this hypothesis job satisfaction was measured through items 38 and 41 of the MLQ questionnaire. A Pearson product-moment correlation coefficient was computed to assess the relationship finding a significant association $(r=0.42, N=26, p<0.05)$. A chisquare value of $19.15, p<0.0001$ was generated for high transformational leadership style, 
suggesting that the relationship may in fact possess an extremely strong positive association. The null hypothesis was consequently fully rejected.

\section{Null Hypothesis Three}

There is no significant relationship between sports coordinators who exhibit transformational leadership and perceived leader effectiveness from coaches.

The hypothesis measured perceived leader effectiveness based on responses to questions 37, 40, 43, and 45 from the survey questionnaire. Rejection of the null hypothesis occurred according to a Pearson coefficient of $r=0.49$ representing a significant relationship between the variables. The probability associated with the chi-square statistic of 15.48 was less than .0001 and indicates there is an extremely strong positive relationship between transformational leadership of sports coordinators and coaches' extra effort. Results were replicated according to chi-squared with Yates correction $(r=12.54, p<0.0004)$ and Fisher's exact test $(p=0.00021)$.

\section{Transformational Leadership Sub-Scales}

$H_{o} 4$ : There are no significant relationships between sports coordinators who exhibit idealised influence (attributed) behaviours and the organisational outcomes

$H_{o}$ 5: There are no significant relationships between sports coordinators who exhibit idealised influence (behaviours) behaviours and the organisational outcomes

$H_{o} 6:$ There are no significant relationships between sports coordinators who exhibit inspirational motivation behaviours and the organisational outcomes

$H_{o} 7:$ There are no significant relationships between sports coordinators who exhibit intellectual stimulation behaviours and the organisational outcomes 
$H_{o} 8$ : There are no significant relationships between sports coordinators who exhibit individualised consideration behaviours and the organisational outcomes

The above hypotheses were all related to the five subscales of transformational leadership. These dimensions include: idealised influence (attributed), idealised influence (behaviour), inspirational motivation, intellectual stimulation, and individualised consideration. Results from the Pearson correlation test are shown in Table 6, indicating a number of significant relationships.

Null hypothesis four postulated that idealised influence (attributed) would have no relationship between any of the three dependent variables. Pearson correlation scores revealed that the leadership behavior may in fact be significantly associated with job satisfaction $(r=$ $0.48, p<0.05)$, extra effort $(r=0.51, p<0.05)$, and perceived leader effectiveness $(r=0.62$, $p<0.01)$. The chi-square values for job satisfaction of 8.55 and perceived effectiveness $(r=$ 7.80) indicated very significant positive relationships $(p<0.01)$. The leadership behavior, however, did not appear to have a significant positive relationship with extra effort in either of the chi-squared $(r=2.16, p=0.14 ; r=1.13, p=0.29)$ or Fisher's $(p=0.29)$ tests. Thus, $\mathrm{H}_{0} 4$ could only be partially rejected, as findings were not sufficiently conclusive for extra effort.

The fifth hypothesis, $\mathrm{H}_{0} 5$, tested the behavioural construct of idealised influence and produced different outcomes to the above. A Pearson product-moment correlation coefficient was computed to assess the relationships, finding no significance between the behaviour and job satisfaction $(r=0.32)$ and extra effort $(r=0.32)$, although a coefficient of $r=0.40$ for perceived effectiveness was significant at the p-value of 0.05 . Further chi-squared tests supported the finding that high scores of idealised influence (behaviour) may not be related to coaches' extra effort $(r=0.96, p=0.33)$. On the other hand perceived effectiveness $(r=9.91)$ and job satisfaction $(r=10.40)$ chi-squared scores were very significant $(p<0.01)$. Once again 
$\mathrm{H}_{\mathrm{o}} 5$ could only be partially rejected, as there appeared to be no correlation with coaches' extra effort.

Results from the tests on the sixth hypothesis were contradictory once again. According to the Pearson correlation coefficients for job satisfaction $(r=0.41)$, perceived effectiveness $(r=0.56)$ and extra effort $(r=0.44), \mathrm{H}_{0} 6$ could be fully rejected. Chi-square values of 11.92, $p=0.0006$ (job satisfaction) and 16.34, $p<0.0001$ (perceived effectiveness) similarly reported that it is extremely unlikely the correlations between the two dependent variables and inspirational motivation is due to chance. Nevertheless, chi-squared, chisquared with Yate's, and Fisher's exact tests all reported no significant association with extra effort. Therefore, $\mathrm{H}_{\mathrm{o}} 6$ can only be rejected for two out of the three organisational outcomes.

Null hypothesis suggested that intellectual stimulation behaviours of sport coordinators are not related to perceived leader effectiveness or coaches' job satisfaction and extra effort. Congruent with the previous leadership dimensions, chi-square values of 5.58 (perceived effectiveness) and 10.40 (job satisfaction) indicate significant associations; extra effort was not significant according to p-value scores above 0.05 for all three chi-squared test variations. The Pearson correlation test remarkably produced opposite results as extra effort had the only significant association $(r=0.44, p<0.05)$. Mixed results between statistical measures mean that $\mathrm{H}_{0} 7$ cannot be fully rejected, despite chi-square scores revealing significant associations between the supposed effectiveness of leaders and coaches' job satisfaction.

Lastly, $\mathrm{H}_{0} 8$ stated that individualised consideration behaviours would not affect organisational outcomes for followers. The null hypothesis was fully supported for perceived leader effectiveness and extra effort according to Pearson correlation coefficients $(r=0.29, r$ $=0.15)$ and chi-square values $(r=2.33, r=0.49)$ both being below desired significance. On 
the other hand, chi-square analysis revealed a very strong positive relationship $(p<0.01)$ for job satisfaction.

\section{Transactional Leadership Behaviours}

\section{Null Hypothesis Nine}

$H_{o}$ 9: There is no relationship between sports coordinators who exhibit transactional leadership and coaches' extra effort.

A two-tailed Pearson correlation test was initially conducted revealing a significant correlation between the two variables $(r=.53, N=25, p<0.01)$. The chance that transactional leadership may cause greater levels of effort in followers was not quite significant in chisquared tests $(r=3.34, p=0.07)$. Although findings indicate that there may be a significant relationship in the population of Wellington secondary school sport coordinators, $\mathrm{H}_{\mathrm{o}} 1$ was only partially rejected as it appears that high levels of the transactional leadership style as a whole may not lead coaches to exert any extra effort.

\section{Null Hypothesis Ten}

$H_{0}$ 10: There is no significant relationship between sports coordinators who exhibit transactional leadership and coaches' job satisfaction.

Results from all statistical measures provide full support for the hypothesis. A Pearson product-moment correlation coefficient of 0.33 ; a chi-square value of 2.60 and 1.46 with Yate's correction; and Fisher's exact score all produced p-values $(0.11,0.23$, and 0.24$)$ much higher than the stated significance level of 0.05 in the relationship between transactional leadership and coaches' job satisfaction. It can be stated sports coordinators who exhibit all an overall transactional style of may not lead coaches' who are satisfied in their roles. 


\section{Null Hypothesis Eleven}

$H_{0}$ 11: There is no significant relationship between sports coordinators who exhibit transactional leadership and perceived leader effectiveness from coaches.

Null hypothesis ten postulated that transactional leadership would have no relationship with perceptions of effectiveness. Pearson correlation scores revealed that the leadership style does not quite possess a significant association $(p<0.05)$ with the dependent variable $(r=0.37)$. Chi-squared analysis resembled similar results with scores of 2.48 ( $p=$ $0.12)$ and $r=1.39(p=0.24)$ with Yate's correction. Lastly, Fisher's exact score provided a p-value of 0.24 . All statistical measures conclude that $\mathrm{H}_{\mathrm{o}} 10$ is fully supported.

\section{Transactional Leadership Sub-Scales}

$H_{0}$ 12: There is no significant relationship between sports coordinators who exhibit contingent reward behaviours and the organisational outcomes

$H_{0}$ 13: There is no significant relationship between sports coordinators who exhibit active management-by-exception behaviours and the organisational outcomes

$H_{0}$ 14: There is no significant relationship between sports coordinators who exhibit passive management-by-exception behaviours and the organisational outcomes

The above hypotheses were all related to the three subscales of transactional leadership. These dimensions include: contingent reward, management-by-exception (active) and management-by-exception (passive). Results from the Pearson correlation test are shown in Table 6, indicating a number of significant relationships.

Null hypothesis twelve assumed that contingent reward behaviours would not elicit an influence on any either coaches' job satisfaction, extra effort, or their perceived effectiveness as a sport coordinator. Firstly, results imply that this behavioural dimension has no significant 
association with job satisfaction. The lack of statistical significance was indicative throughout the Pearson correlation coefficient $(r=0.31, p>0.05)$, chi-squared $(r=3.72, p=$ $0.54)$, chi-squared with Yate's correction $(r=2.32, p=0.13)$ and Fisher's exact score of 0.11 . The second variable, perceived effectiveness, produced an $r=0.42, p<0.05$ in the Pearson's tests indicating significance. The chi-squared test did not quite reveal similar significance though with a p-value of 0.052 , signifying that the relationship from MLQ responses may in fact be due to chance.

Most notably, contingent reward behaviours appeared to be the only leadership dimension in the nine-factor model to significantly affect coaches' extra effort though. A Pearson coefficient of 0.63 was very significant $(p<0.01)$; as were chi-squared $(r=4.57, p=$ $0.03)$ and Fisher's exact values (0.049).

In sum, $\mathrm{H}_{\mathrm{o}} 12$ was fully supported in relation to job satisfaction; partially supported for perceived effectiveness; and fully rejected for extra effort. This illustrated that contingent reward behaviours may be employed by sport coordinators to ensure a satisfied following. Moreover, coordinators wishing to have their coaches' exert greater effort in their provision of sport may find utility in exhibiting contingent reward practices.

Null hypothesis thirteen postulated that management-by-exception (active) would have no relationship with organisational outcomes. Results imply that this leadership behaviour is not significantly $(\mathrm{p}<0.05)$ correlated to any of the organisational effectiveness measures. The findings were consistent for perceived leader effectiveness $(r=0.29$, job satisfaction $(r=$ $0.12)$ and extra effort $(r=0.26)$ throughout the Pearson correlation analysis. This was no different in the chi-squared, chi-squared with Yate's correction and Fisher's exact tests for each organisational outcome, meaning full support of $\mathrm{H}_{0} 12$.

The other management-by-exception dimension (passive) similarly could not prove the effects on the any of the organisational outcomes were not down to chance. Pearson 
correlation values ranged from $r=0.04$ to $r=0.33$ and were therefore not statistically significant at the 0.05 level. The explanatory power of discrepancies between high and low frequencies were also very weak in chi-squared tests $(p=0.67 ; p=0.62 ; p=0.74)$ respectively for perceived effectiveness, job satisfaction and extra effort. Consequently the null hypothesis $\left(\mathrm{H}_{\mathrm{o}} 14\right)$ was fully supported.

\section{Laissez-Faire Leadership}

\section{Null Hypothesis Fifteen}

$H_{0}$ 15: There is no significant relationship between sports coordinators who exhibit laissez-faire leadership and coaches' extra effort.

A total of four correlational tests were performed to find support for the above null hypothesis, or reject it in favour of an alternate. Firstly, a Pearson correlational test was performed on the data illustrating no statistical significance $(\mathrm{p}<0.05)$ between the laissezfaire style and coaches' extra effort $(r=0.34)$. The findings were replicated in further tests with values of $r=2.16, p=0.14$ for the chi-squared test; a score of $r=1.13, p=0.29$ when performed with Yate's correction; and a Fisher's exact score of 0.29. Taken together the results provide full support for $\mathrm{H}_{0} 15$, suggesting no significant relationship appears between sports coordinator who exhibit laissez-faire leadership and coaches' extra effort.

\section{Null Hypothesis Sixteen}

$H_{0}$ 16: There is no significant relationship between sports coordinators who exhibit laissez-faire leadership and coaches' job satisfaction.

When testing this null hypothesis the statistical significance for the relationship varied between Pearson and chi-squared tests. A very significant correlation value $(r=7.49, p<$ 0.01) was exemplified through the chi-squared tests between low and high frequencies of each variable. A Fisher's exact score of 0.01 supported this relationship. However, when 
measuring the overall scores through the Pearson test, the coefficient value was very weak and suggested no statistical significance $(r=0.06)$. It was concluded that $\mathrm{H}_{\mathrm{o}} 16$ was only partially rejected.

\section{Null Hypothesis Seventeen}

$H_{0}$ 17: There is no significant relationship between sports coordinators who exhibit laissez-faire leadership and perceived leader effectiveness.

The final hypothesis anticipated that sport coordinators' self-reported frequencies of laissez-faire leadership would not be significantly correlated to perceived leader effectiveness. Statistical significance at the $p<0.05$ level was not achieved in any of the Pearson, chi-squared, chi-squared with Yate's correction or Fisher's tests $(r=-0.05 ; r=1.33$; $r=0.58, P=0.43)$ respectively. The Pearson test did reveal a negative relationship suggesting (although without significance) that as sport coordinators' frequency of exhibiting laissezfaire leadership rises, correspondingly their perceived leader effectiveness ratings will drop. Findings did not provide any indication to otherwise disprove $\mathrm{H}_{\mathrm{o}} 17$, thus the hypothesis was fully supported.

In relation to possible correlations between the three organisational outcomes result revealed an extremely significant positive relationship between perceived leader effectiveness and coaches' job satisfaction. A chi-squared value of 12.57 also produced a p-value of 0.0004 . Conversely this was the only significant interplay between the dependent variables.

\section{Research Question 3}

The third and final research question that will be presented in the results questioned, "What leadership behaviours do sports coordinators identify as most valuable to the effectiveness of their school sport provision?" Responses were gathered from the semistructured interviews and provided both a diverse and diverging range of opinions. 
When interviewees were asked to broadly describe what they believed makes a good sport coordinator, it was commonly stated that you must willing to go above and beyond the cause. A number of key behavioural items arose from interviews including passion, flexibility, and dedication. Sport coordinator 2 summarised this theme,

SC2: "I think they've got to have a passion for what they do... they've got to be prepared to give up their own time... you've got to be pretty flexible in terms of staff... and expertise in sport".

Comparably Sport coordinator 1 outlined how those in the role need to be able to assume a variety of roles, often beyond the job description:

SC1: "They need to take responsibility and take leadership of any sort of role or just whatever is going on, they need to take control but be able to relate to everyone as well and listen to everybody else".

Interviewees were further probed to discuss the specific leadership behaviours that are valued in the role. Overall, all four coordinators were zealous in their views that leadership is crucial for sports coordinators to effectively lead and manage school sport. Sport coordinator 4 claimed, "Leadership, it's definitely a huge thing. I think it would be pointless doing sport without it". Without much knowledge on leadership literature all sport coordinators were emphatic that dimensions of transformational leadership are a necessity within the school sport environment.

Firstly, before specifically explaining the 'New Leadership' theory and its subscales to respondents, all coordinators had either loosely heard of transformational leadership or had been encouraged to adopt its behaviours. When asked what leadership behaviours were appreciated and useful for their roles a number of transformational constructs were alluded to 
and specifically stated. Themes of mentoring, inspiring and seeking to achieve group goals emerged in the answers; all of which underpin the transformational theory.

The ability of sport coordinators to build commitment for the department's vision and goals was exhibited in Sport coordinator 2's response. Throughout the interviews both Sport coordinators 2 and 3 repeatedly stated that their delivery of sport to students needed the input of staff that want to achieve a common goals. This was displayed when Sport coordinator 2 stated, "We've got a lot of student coaches who we couldn't function without". The ability to assign coaching, management and administrative to volunteers who can commit to their vision of sport was integral. Moreover, an effective leader was,

SC2: "Someone who' able to pick people up and carry them with them to an end product. Then if you've got four or five people heading in the same direction then also a wave starts and you get a few more, and a few more, and it snowballs in! That would be my idea of leading the way. I often say to get people involved you have to get them to stop thinking about having to do something, and think about wanting to do it”.

The above quote can also be assumed to provide a clear example of the 'falling dominoes effect' (Bass \& Avolio, 1994) in the secondary school sport setting. Taken together the quotes can be seen to express that idealised influence and inspirational motivation are esteemed leadership behaviours.

Likewise, three of the four interviewees valued innovation and being responsive to the needs of students, the school, and sport programmes in general. In order to impart such organisational change, the transforming nature of the 'New leadership' theory to alter existing structure and reform practices (Bass, 1999; Yukl, 1989) has been said to have utility. This was no different for leaders in the secondary school sport setting. Responses suggest that coordinators have to be willing to change and not always accept the traditional ways of 
operating; they must question assumptions and always attempt to implement the best options. Quotes representing this belief are presented below.

SC4: “A leader would be somebody who'd be going out and being innovative and actually saying 'well you know I can think of a better way of doing this or we've got an issue here".

SC2: "We've always done it this way! I actually saw a quote the other day that said 'We've always done it this way - the seven most expensive words in business'. I'm going to put it up there on my wall somewhere cause you hear it an awful lot... but now it's actually like no we've actually done this before and I don't think it works and I guess you need to put both of these together to really understand and get people on the same page".

SC3: "If it's not broken don't fix it isn't always a good thing".

Achieving the desired changes, however, can be very difficult. It is vital to be diplomatic and pick your battles when trying to induce change, according to Sport coordinator 2. Further responses advise that relationship maintenance provides the foundation for all sporting codes and practices; you must be relatable and able to work with a number of different parties to gain support. For example, Sport coordinator 1 alluded to the efficacy of such individualised consideration with each relationship as, "if one falls over it feels like everything falls over. So it's about making sure that every single relationship that you've got is getting the attention it needs, and the help and the support" (Sport coordinator 1).

On the other hand, responses indicate that poor leaders would be more aligned with leadership dimensions at the opposite end of the transformational-transactional continuum. When asked, "What do you think makes a poor leader in the coordinator role? Can you provide any traits or behaviours?" it was stated that such a person would act selfishly, only be willing to complete the bare minimum requirements, and avoid making changes despite the 
apparent benefits. Combined these behaviours can be seen to loosely represent the nonleadership construct, laissez-faire.

Following the above questions a handout was presented to each sport coordinator that guided them through each dimension within the Full Range Model of Leadership (Appendix D). Respondents were then asked to discuss which behaviours are prevalent within secondary school sport and provide examples; additionally, sport coordinators were questioned on which behaviours they believed to be most effective or ineffective. Responses were collated in accordance to the specific leadership subscales and are presented below.

In similar light to the unguided question on ineffective leadership, transactional leadership and more fervently laissez-faire styles were deemed to be out-dated and ineffectual.

During the interview with Sport coordinator 3 the most ineffective style was declared to be "Laissez-faire of course! You just can't do a job like that". In regards to the specific actions (or lack off) inherent within the style, "avoid accepting responsibilities, absent when needed... the whole ethos of it goes against the values of the school" (Sport coordinator 4). The changing nature of desirable leadership styles was apparent in this setting. Coordinators believe that they cannot sit back and assume school sport will be ever present; rather, you must get the most out of available resources. Sport coordinator 4's summary "I don't think the last one has a place anywhere anymore. You can't do that anymore; it's the old way isn't it! So I'd write that off' clearly illustrates the need for pragmatic leadership.

Passive management-by-exception may also result in negative consequences. According to Sport coordinator 1, this can also be ineffective "somewhat, if you stand by too much". Instead, the coordinator believed you have to be in constant communication and provide support networks for coaches. Sport coordinators 3 and 4 also held this principle that was exemplified in the following response, 
SC4: "Like you said the Passive Management, rather than anticipate an issue 'cause you can see where things are going if you don't deal with it until it happens... something might happen by that time and it could have been avoided. Or something has happened that you can't change and it's too serious so might have long-term impacts".

Overall responses point to the fact that traditional leadership styles may no longer hold a place for secondary school sport coordinators. Nevertheless, all management roles require observation of followers and correctional action if necessary. Active management-byexception also appears to have function in secondary school sport. This behaviour is commonly referred to as "leaders [who] actively monitoring the work of followers and making sure standards are met" (Appendix D). In the studied context sport coordinators are required to fill numerous coaching roles within their sporting codes offered. As such, it is imperative that those people who assume paid or voluntary jobs are competent and continually provide effective coaching. Interviews results reinforce this point, yet monitoring and interventions must be subtle so as to retain coaches. Sport coordinator 3's quote exhibited that,

SC3: “Again we have to do this quite gently because we don't pay anybody. So unless we do this gently people go, so we have to sort of be there and [say] 'great job!'

There's no way we could do that with a strong approach but we certainly still have to keep the standards up as far as looking after the girls and making sure they're looked after well".

The above response also illustrates the need to praise followers for their contributions. Such praise constitutes the verbal aspect in contingent reward behaviours, which was deemed an effective behaviour by sport coordinators in the semi-structured interviews. Responses indicate that it is important to reward coaches' efforts with any available resource, whether 
this be monetary or through personal recognition. School sport literature suggests that the majority of coaches did not receive paid employment (SPARC, 2011; Sport NZ, 2008) and this was found to be consistent according to Sport coordinator 4 who said, "we're working in and environment where there's very little money to go around so there's very little you can offer for rewards". Nevertheless, the capacity to provide praise and recognition is unlimited and provides an effective alternative to retain coaches and keep them motivated (Sport coordinator 3, 4). A simple pat on the back or acknowledgment in the school newsletter can suffice.

Despite the benefits associated with contingent reward behaviours, sport coordinators strongly encourage the use of transformational leadership. An effective coordinator will influence followers to transcend their own self-interests for those of the school as, "it's not about you're going to get a reward at the end, it's more about being a part of something" (Sport coordinator 4). All four participants converged on the notion that the philosophy of transformational leadership is the dominant and preferred way of leading their respective sport departments.

Responses provided support for each of the five subscales and sport coordinators suggest that these can be utilised to achieve the best performance from followers. Firstly, three of the four sport coordinators specifically stated that inspirational motivation is an effective behaviour. Sport coordinator 2 argued this to be the single most effective behaviour in the nine-factor model, specifically for its purpose to gain respect and subsequently a following. Conversely, Sport coordinator 3 could not single out the 'most valuable behaviour' stating, "No I don't think any one of the behaviours stands out. I think it's a very big combination of all that [pointing to transformational handout]". Yet the coordinator did state that individualised consideration, idealised influence and inspirational motivation are essential for getting volunteers to contribute and keeping them around. The other three 
respondents also touched on the valued outcomes of these behaviours. Finally, encouraging debate around the best methods for providing sport to students through intellectual stimulation was conceived as a valuable part of Sport coordinator 4's role. 


\section{Chapter V: DISCUSSION AND CONCLUSION}

Prior chapters introduce the study, provide a comprehensive literature review on the theoretical and empirical foundations of the 'New Leadership' model, outline the methodological approach used for this study, and provide the results from the MLQ survey and semi-structured interviews. This chapter will present a summary of key findings and implications for sport coordinators while also confirming, or not, the applicability of previous theoretical extant theoretical frameworks and findings. In a final section, the practical implications and limitations of the research will be considered with an ensuing discussion on how future research could expand this field of knowledge.

\section{Research Question 4}

Confirming or not the applicability of previous studies frameworks and findings on transformational, transactional, and laissez-faire leadership and organisational effectiveness to the context of Wellington Secondary School Sport'

The Full Range Model of Leadership has received empirical focus predominantly in the business setting, whereas this study's context has been scantly researched. Only a handful of books and articles published in academic journals have explored transformational leadership in New Zealand organisations (Bass, 1985; Bass \& Rigio, 2006; Bass et al., 1987; Parry \& Proctor-Thomson, 20003; Singer, 1985; Singer \& Singer, 1986, 1990), and school sport administrators in the U.S. (Burton \& Peachey, 2009; Doherty, 1986; Doherty \& Danylchuk, 1987; Kent \& Chelladurai, 2001; Peachey \& Burton, 2010; Weese, 1995; Zhang et al., 2004). The lack of research in these settings is interesting despite key authors recently promoting transformational leadership as the most relevant behaviour for sport managers (Gilbertson et al., 2000; Soucie, 1994; Weese, 1994) and educational administrators (Leithwood \& Jantzi 2005). 
This current research study's objective to compare and contrast previous theoretical frameworks and findings is not to conclude cause and effect, rather, it is intended to determine the currency of the model for the New Zealand school sport sector. This aim becomes more appropriate as the thirty years of research on the model has promoted transformational leadership to be the most desirable (Den Hartog et al., 1999; Leong \& Fischer, 2010; Singer, 1985) and effective (Bass \& Riggio, 2006) style in a range of organisational settings (Bass, 1997; Yukl, 1989). Meta-analyses of literature (Bass, 1999; DeGroot et al., 2000; Lowe et al., 1996; Judge \& Piccolo, 2004; Wang et al., 2011) has also shown that transformational and transactional leadership may positively predict a range of organisational outcomes - such as follower's job satisfaction, the extra effort of colleagues, collaborators, volunteers, followers etc. and perceived leader effectiveness - that contribute to greater performance and effectiveness.

Despite a widespread view that transformational leadership offers universal practicality (Bass \& Riggio, 2006), research has shown that its applicability and effectiveness may be moderated by situational contingencies. For example, Leong and Fischer (2010) revealed that the values, beliefs and norms that underpin national cultures also impact leadership preference and effects. When discussing the model's value for sport managers, Bass himself has acknowledged that situational contexts impact the efficacy of the model (Weese, 1994). Empirical research employing the MLQ instrument has produced mixed or ambiguous findings in the sporting and school fields. The conflicting results "suggest that more research is needed to test this theory in sport settings" (Aminuddin, 1998, p. 171). Correspondingly, the following discussion will provide points and counter-points between statistical and behavioural significance of this study's findings in relation to earlier literature. 


\section{General Leadership Profile}

Bass (1999) proposed that the Full Range Model of Leadership predicates that leaders display both transformational and transactional behaviours, but that each leader's profile will be characterised by one having a higher frequency. Bass also stated that the success of attempts to improve leadership depends on reducing management-by-exception behaviours whilst also increasing the regularity of transformational behaviours (2000). Furthermore, in relation to organisational outcomes, transformational behaviour tends to be the most successful, followed in order of effectiveness by contingent reward, active management-byexception, passive management-by-exception, and laissez-faire (Avolio \& Bass, 1990; Bass, 1997).

The leadership profiles of Wellington secondary school sport coordinators were measured through self-rater versions of the MLQ Form 5X (Avolio \& Bass, 2004). The findings provided overall support for the theoretical propositions of the Full Range Model stated above. All of the 26 respondents perceived their own leadership style to be dominantly transformational. Results implied that sports coordinators display distinctly more selfreported transformational leadership $(M=3.01, S D=0.57)$ than transactional $(M=1.86, S D$ $=0.51)$ or laissez-faire leadership behaviours $(M=0.47, S D=0.66)$, thereby providing support for Bass' proposed continuum (2000). Comparable to Bass' (1999) claim that leaders can simultaneously exhibit a number of leadership behaviours, both correlational analyses and interview responses illustrate that sport coordinators exhibit a combination of the nine leadership factors. Sport coordinator 2 claimed:

SC2: "All three styles are covered within the spectrum of school sport", although transformational leadership was more valued and sought. 
The sport coordinator characteristics provide a similar pattern when compared to Bass' (1996) large sample study, and Lowe and colleagues' meta-analysis (1996). All of these studies reported the highest mean frequency or usage scores in the set of transformational behaviours, closely followed by transactional leadership behaviours. By contrast, laissezfaire was only observed 'Once in a While'.

A comparison of the observed leadership behaviour profiles to similar settings and contexts demonstrates similar patterns to those of New Zealand company managers (Bass, 1985; Singer, 1985; Singer \& Singer, 1986), and also American school athletic directors (Doherty, 1997; Doherty \& Danylchuk, 1996; MacDonald, 2012; Manning, 2012; Miyashiro, 2007; Rouff, 2007). Wellington secondary school sport coordinators were similarly characterised by the MLQ instrument to be predominantly transformational leaders that also frequently utilised the provision of contingent rewards. Additionally, interview participants spoke of their leadership styles more frequently and favourably - in a manner identifying resemblance to the '5 I's' and contingent reward.

It was interesting to see that contingent reward behaviours (manifest as scores: $M=$ $2.87, S D=0.74)$ were displayed to a similar level of frequency as scores for transformational behaviours. Furthermore, Pearson correlation scores showed that whilst contingent reward behaviour displayed significant positive associations with scores of four of the five transformational constructs, it was not associated with either management-by-exception behaviour. These findings may be explained by either of the following narratives.

Firstly, the findings could be seen to provide evidence of the augmentation effect (Bass, 1985; Yammarino \& Bass, 1990). This stipulates that contingent reward is important for establishing trust and providing the foundation for transformational leadership to follow. More specifically, Bass (1998) stated that "consistent honouring of transactional agreements builds trust, dependability, and perceptions of consistency with leaders by followers, which 
are each a basis of transformational leadership" (p. 11). This present study may also provide corroborative support for the effect (Avolio, 1999; Bass \& Avolio, 1994; Parry \& Proctor-Thomson, 2003) despite Bass and colleagues' (2003) study contrarily providing no evidence for the augmentation effect.

Alternatively, contingent reward may actually be better represented as a transformational dimension of leadership according to Goodwin and colleagues (2001). Their study attempted to develop a theoretical framework to explain why recent empirical results, such as those of this study, have linked contingent reward to transformational rather than transactional leadership. Goodwin's study concluded that the MLQ items measuring the construct might be further divided into explicit and implicit exchange behaviours. The former is associated with clarifying expectations associated with rewards, whereas the latter may be more related to transformational leaders in as much as they guide subordinates and others to fulfil implicit expectations without overtly negotiating with them. Qualitative responses from this study may be seen to further elucidate the nature of such relationships. For example, whilst coordinators stated that the majority of coaches are volunteers and do not enter in to contracts with coordinators, this not did not prevent coordinators from providing appropriate recognition and praise for volunteer coach efforts.

\section{Leadership subscale frequencies and means}

Following the examination of general patterns in the leadership profiles of sport coordinators, it was important to compare and contrast the self-reported behaviours through the operational measures such as the frequencies and mean scores of the nine leadership behaviours. The findings are firstly presented to illustrate the similarly high occurrences of transformational leadership behaviours when compared to school sport leaders from previous literature, such as Doherty (1997) and MacDonald (2012). Following this, it is shown that reported frequencies of the three transactional dimensions varied significantly for individual 
coordinators, as management-by-exception was reported far less than contingent rewards.

Lastly, the mean scores for passive and non-leadership styles were shown to be much lower in the school sport environment when compared to other organisational settings. The following discussion proceeds to provide possible explanations for this study's findings based on existing theoretical frameworks and assumptions.

A deeper exploration of leadership subscales revealed that no single transformational dimension occurred more often than others. Instead all five behaviours were displayed 'Fairly Often'. Quantitative scores revealed a minor difference $(M=0.38)$ between the lowest (intellectual stimulation, $M=2.85$ ) and highest (individualised consideration, $M=3.23$ ) mean scores for transformational constructs. Moreover, the five transformational behaviours were perceived to occur regularly and were evenly spread around the mean score of 3.01. The findings were also consistent with previously reported MLQ frequencies (Doherty, 1997; Doherty \& Danlychuk, 1996; Manning, 2012; Rouff, 2007; Singer, 1985), and there were minimal differences between mean scores of the ' 5 I's', suggesting that all of the constructs are also commonly used by Wellington school sport leaders.

Generally, the findings from the quantitative results imply that sport coordinators are seen to utilise all transformational behaviours regularly, if not habitually. Interview responses were interpreted to supplement the MLQ data, explaining that sport coordinators must employ different behaviours to favourably influence the various parties and stakeholders. Notably consistent through the interviews conducted was the participants' belief that sport leaders are required to consciously modify their leadership styles according to the situation and/or person. For instance, Sport coordinator 1 claimed:

SC1: "You can't be one person and get along with everyone.

You've got to be able to change your style and approach people differently". 
The notion of a fluent and adjustable leadership style was also observed as being valuable, as sport coordinators indicated that they are responsible for providing a vast amount sporting activities and for maintain a variety of internal and external relationships.

The self-reported consistency of use of all transformational behaviours by Wellington secondary school sport coordinators was in itself, pleasing for the secondary school sport sector, given that Bass (1985) has declared the best leaders use all dimensions of leadership behaviour in creating success. The semi-structured interviews added further to the researcher's ability to explain observed behaviours, suggesting for example, that coordinators were consciously modifying their styles in response to situational contingencies. The ability of leaders to be flexible and adaptable to their constituents was also characteristic of effective leader behaviour found elsewhere (Bass et al., 2003; Lowe et al., 1996). Overall, the transformational leadership styles were reported to be high, stable, and adaptable; and the literature indicates that this may serve sport coordinators well in promoting change and positively influencing their school sport programmes.

Although the sport coordinator's Full Range Model of Leadership profiles reported transformational and contingent reward characteristics comparable to previous studies, there were some differences in the mean scores self-reported for transactional and laissez-faire styles.

Most notably, there were statistically significant differences between the three transactional constructs, as contingent reward $(M=2.87)$ arose more commonly than management-by-exception (1.80 active and 0.90 passive). The qualitative behavioural responses corroborated the statistical scores, as manifested in participant assertions that coordinators should reward coaches whenever possible, but never sit back and fail to intervene when problems arise. These findings provide another parallel to the transactional behaviours of American athletic directors from inter-university athletic (Doherty, 1997; 
Doherty \& Danylchuk, 1996; Manning, 2012; Rouff, 2007) and high school (MacDonald, 2012; Miyashiro, 2007) athletic programmes. Overall, the findings suggest that sport coordinators may demonstrate similar sport leadership profiles regardless of the national differences or settings.

When comparing the leadership behaviours of sport coordinators in this study to the behaviours of individuals in others organisational contexts, there are fewer frequent occurrences of management-by-exception (active or passive) and laissez-faire. For instance, an early meta-analysis of MLQ studies (Lowe et al., 1996) collated an average managementby-exception score from studies across a diverse range of private and public organisations in various countries. The collective $M=2.32$ was much higher than the reported score of $M=$ 1.35 for this study. Similarly, Bass and colleagues' (1996) large sample study and Singer and Singer's studies of New Zealand company managers (1985; Singer \& Singer, 1986) reported much higher management-by-exception frequency means around 1.8-2.2 for both active and passive behaviours.

The lower levels of passive and non-leadership behaviours in the school sport context may be due to the different organisational structure and requirements when compared to business settings. For example, leaders in private companies will often have explicit contracts with subordinates outlining job requirements and expectations. Interventions and correlated actions may therefore only be necessary retrospectively when performance measures are not met. Conversely, coaches are responsible not only for performance outcomes (win/loss ratio etc.), but also the enjoyment and safety of students, as reported in participant interviews. Thus, sport coordinators may be required to be more pragmatic and responsive to their coaches because as sport coordinator 1 said: 
SC1: "if one [relationship] falls over, it feels like everything falls over. So it's about making sure that every single relationship that you've got is getting the attention it needs, and the help and the support."

\section{Summary of Leadership Profiles}

Leadership behaviour frequencies self-reported in this study were very similar to Manning's (2012) self-perceived leadership profiles of NCAA athletic directors. Mean scores were much higher in the transformational and contingent reward dimensions, whilst also reporting statistically significantly lower means for management-by-exception and laissezfaire when compared to studies that employed rater-versions of the MLQ (Bass et al., 1996; Doherty \& Danylchuk, 1996; Doherty, 1997; Lowe et al., 1996; MacDonald, 2012; Miyashiro, 2007).

Comparable research findings suggest that Wellington secondary school sport coordinators also exhibit the more favourable leadership behaviours identified by Bass's (1985. Conversely, one could argue that the high frequencies of transformational and contingent reward, in conjunction with low passive leadership rates, may not reflect reality. Manning (2012) believed that the similar self-reported profiles of their study might have been biased due to the methodological nature of data collection. This may signify that the selfreported leadership behaviours may be prone to distortion or exaggeration.

The possibility that sport coordinators overstated or overemphasised their leadership behaviours may be further explained through prior theoretical analysis of self-report questionnaire instruments as data gathering tools. Such surveys have been shown to produce 'inflated' results, as respondents perceive themselves to be engaging in a greater frequency of desirable behaviours (Crampton \& Wagner, 1994; Kolb, 1995; Podsakoff, MacKenzie, Lee \& Podsakoff, 2003). This phenomenon has also been identified elsewhere in organisational 
behaviour research (Spector, 1994) and more specifically in reviews of MLQ research (Avolio, Yammarino \& Bass, 1991; Yukl, 1989).

It is important not to conflate the practical and statistical significance of apparent Wellington secondary school sport coordinator leadership profiles, or to overstate the findings from each stage of the study. On the one hand, this study has been successful in avoiding the biases of mono-methods through its second stage semi-structured interviews, which, in this study, provided support for the leadership characteristics surfaced via the quantitative operational measures. Nevertheless, the self-rater version of the MLQ may have generated frequencies that do not reflect the actual behaviours of sport coordinators. In addition, the researcher is aware of the need to make explicit the methodological weakness arising from the small sample of participants that effectively limit the reliability of results and findings related to leadership behaviours, and which, in turn, impact the generalizability of findings across New Zealand.

\section{Relationships between leadership behaviours and organisational effectiveness}

In addition to determining the leadership behaviour profiles of Wellington secondary school sport coordinators, the present study also sought to evaluate through the MLQ (5X Short-Form) how leaders influence coaches' job satisfaction, extra effort and perceived leader effectiveness. Likewise, interviews with sport coordinators were conducted to explore the influence their leadership behaviours have on the various other parties that help contribute to sporting programmes.

Within the present research study, each dependent variable (job satisfaction, extra effort and leader effectiveness) was examined in association with the independent variables (transformational, transactional and laissez-faire) and their respective subscales. Null hypotheses were formulated for observed associations and relationships and were subject to statistical hypothesis testing using Pearson correlation and chi-squared statistics. Results from 
the quantitative method helped develop and inform further lines of inquiry, investigated through subsequent semi-structured interviews. As indicated by Hanson et al. (2005), the semi-structured interviews with sport coordinators provided qualitative data that enabled the researcher to interpret and elaborate on the quantitative statistical relationships that emerged between measures of the different leadership behaviours and, for example, outcome measures related for coaches. Moreover, the qualitative data also shed light and extended the breadth of inquiry to include the various internal and external parties that interact with the sport coordinators. Indeed, the interactions that sport coordinators have with fellow staff members, students, parents and external organisations (e.g., Sport Wellington employees) may equally impact organisational outcomes, performance and effectiveness.

It is therefore important to establish whether knowledge/measures of leadership behaviours can be used to predict such outcomes given that such behaviours and outcomes may also have a positive impact on followers' performance and effectiveness (Bass et al., 2003; Yukl, 1989). In addition, sport managers may be interested to discover whether the findings of other studies in other contexts may hold true in the Wellington secondary school sport setting. Indeed, if specific leadership styles are positively related to followers' satisfaction and efforts, then sport coordinators may have the potential to impact on the quantity and quality of sporting provision.

Several researchers have suggested that use of the transformational-transactional model can be used to predict job satisfaction, extra effort and perceived leader effectiveness (Bass, 1994; Bass \& Avolio, 1994; Bass et al., 2003; Bass \& Rigio, 2006; Northouse, 2012), which may contribute to greater performance and effectiveness. However, whilst empirical studies have provided support for such theorising and predictions (DeGroot, Kiker \& Cross, 2000; Lowe et al., 1996; Judge \& Piccolo, 2004; Wang, Oh, Courtright \& Coulbert, 2011, this study has identified variances across a range of contextual factors. 
The findings of the present study reveal that of the proposed null hypotheses related to the three leadership styles, only $\mathrm{H}_{0} 2, \mathrm{H}_{0} 3$ were fully rejected.

$H_{o} 2$ : There is no relationship between sports coordinators who exhibit transformational leadership and coaches' job satisfaction.

$H_{o} 3$ : There is no significant relationship between sports coordinators who exhibit transformational leadership and perceived leader effectiveness.

Statistical testing of Pearson correlation coefficient scores indicated that only transformational leadership styles of sport coordinators were statistically significantly correlated to coaches' job satisfaction and perceived leader effectiveness $(p<0.05)$. Chisquared tests of association identified the same relationships as also being statistically significant, whereby explanation purely as a result of chance were very low $(p<0.05)$. As a whole, the findings suggest that only transformational leadership behaviour styles that might usefully predict organisational outcomes.

\section{Job satisfaction}

Transformational leadership encompasses behaviours that have been shown to engage in satisfying the higher order needs of followers (Burns, 1978). Transformational leaders communicate and strive to achieve a desirable vision for the future often act selflessly and in the interests of both individuals and groups (Bass, 1999). Bass has suggested (1997) that leadership style may have direct and indirect effects on a number of outcomes valued by subordinates, such as autonomy, pride, and meaning in their work.

Results and findings from this study support these claims in as much as Chi-square analysis indicated that transformational leadership exhibited an extremely positive association $(p<0.0001)$ with coaches' job satisfaction. Likewise, tests of association of job satisfaction with all five transformational leadership subscales, reported chi-square statistics and related probabilities that on the basis of a null hypothesis of no association, were well below a p-value of 0.01 . The statistically significant association/relationship between 
transformational leadership and coaches' job satisfaction was supported by the qualitative data, that is, the comments of interviewee participants. Indeed, sports coordinator interviewee participants were unanimous in their belief that the coordinator role requires them act in ways that show the value and appreciation they have towards their staff, otherwise followers won't be motivated to contribute to the cause.

The findings of this study corroborate with other studies conducted within New Zealand of company managers (Bass, 1985; Singer, 1985; Singer \& Singer, 1986). This possibly signifies that the 'team approach' to leadership inherent in the transformational style offers greater satisfaction to workers in collectivistic and egalitarian cultures like New Zealand (Bass, 1999; Leong \& Fischer, 2010; Ramachandaran \& Krishnan, 2009). Leong and Fischer state that, "in egalitarian contexts, individuals are expected to take care of fellow citizens and show moral integrity and responsible behaviour" (2010, p. 166). It is claimed that dimensions of individualised consideration and idealised influence act to make the followers feel valued and perhaps to transcend their own self-interests for the good of the group, thereby explaining why transformational leadership may be a preferred style of behaviour, and may offer greater satisfaction to Wellington secondary school sport coaches.

Although such findings point to the efficacy of leadership for positively influencing follower satisfaction, Locke (1996) provides a reminder that satisfaction is a multidimensional construct and may not solely determined by the leader or leader behaviours. Voon and colleagues' review of the literature (2011) claimed it has many sub-facets, and that many contextual factors may also impact on job satisfaction, such as job responsibilities, working conditions, colleagues and so forth. The inability of many erstwhile leaders in school sport to impact/affect job satisfaction in followers (MacDonald, 2012; Wallace \& Weese, 1995) may indicate that inducing job satisfaction may be beyond not just their immediate 
control, but beyond their control of external factors. Thus, it would be inappropriate to conclude that leadership is solely responsible for affecting coaches' satisfaction.

\section{Perceived leader effectiveness}

In regards to the other measured organisational outcomes, including perceived leader effectiveness, it was no surprise that transformational leadership was the only style to be significantly correlated. Bass and Avolio (1997) conceived leadership effectiveness to be measured by the capacity to meet followers' job-related needs, represent their group to higher authorities, and contribute to the accomplishment of organisational goals and requirements. Northouse (2012) has theorised that appropriate transformational leadership behaviours can lead can facilitate the goals that define organisational effectiveness. Bass (1985) has long claimed that transformational leaders transcend their own self-interests for those of their followers and the overall group. Therefore, transformational leaders can be seen to possess the capabilities to realise followers' needs, and also act in their best interests when reporting to their superiors. Transformational leadership is also characterised when leaders, in embracing an organisation's vision for the future, are then able to motivate followers to greater levels of performance (Bass \& Riggio, 2006). The requirements of the organisation may thus be realised and often exceeded through this style.

Sport coordinator interviewees spoke of the benefits of adopting a transformational leadership style for attracting and retaining volunteers (Sport coordinators 2, 3, and 4); which they suggested was amongst their most important requirements in the provision of sporting opportunities. Moreover, interviewees expressed a view that effective leaders enacted individualised consideration to stay in constant communication with followers to ensure their needs were being met. Synthesising the quantitative analyses and qualitative findings of this study thus corroborate the effectiveness of transformational leaders in this respect. 
While scholars such as Bass and Riggio (2006) and Northouse (2012) contend that the 'New Leadership' approach is universally the most desirable and effective style for promoting greater organisational performance and success, recent meta-analyses have also illustrated the efficacy of contingent reward behaviours (Bass, 1999; Lowe et al., 1996; Judge \& Piccolo, 2004). This research suggests that sport coordinators who exhibit transformational behaviours may be enacting the most effective style whilst also promoting greater satisfaction for coaches; however, the transformational leadership style may fall short in the pursuit of influencing followers to go above and beyond their expectations.

\section{Extra effort}

A central precept of transformational leadership theory is the ability to inspire followers to set aside their own self-interests and assume those of the organisation (Bass, 1985). It is assumed that by adopting the values and objectives required to achieve the leader's vision of the future, subordinates will then apply themselves more enthusiastically and rigorously in their pursuit of success (Bass \& Avolio, 1994).

However, and by contrast, the statistical analyses conducted in this study did not provide statistically significant evidence that sport coordinators who exhibit transformational leadership could influence coaches' to exert extra effort. Although Pearson correlation coefficients produced significant scores for transformational leadership and its subscales of idealised influence (attributed), inspirational motivation and intellectual stimulation, chisquared tests indicated that these associations may be explained as chance occurrences assuming the null hypothesis to be true. The statistical findings were in direct contrast to Bass's research $(1985,1997,1998,1999)$ and other research studies employing the MLQ on American school sport leaders (Choi, 2006; Doherty \& Danylchuk, 1996; Doherty, 1997; MacDonald, 2012; Miyashiro, 2007). Indeed, results and findings corresponded to DeGroot et al.'s meta-analysis (2000) that found transformational leadership cannot predict extra 
effort in followers. However, the lack of evidence, or apparent inability of transformational leaders to influence extra effort in this study, may be due to the situational context and organisational structures of Wellington secondary school sport.

Unfortunately, unlike many NCAA organisations, New Zealand secondary school sport coordinators, and their schools, lack the financial resources to employ paid coaches and administrators (Adamson \& Handcock, 2013; Marshall \& Hardman, 2000), and are thus are dependent on voluntary contributions from teachers, students, and parents. As stated by sport coordinator 4, ever-increasing demands on staff members mean:

SC4: "Teachers just don't seem to have the time to do it all the time... So it's always a struggle trying to get teachers involved."

It is presumed that whilst the sport coordinator may be able create a desire and willingness to volunteer, that, for example, teachers as volunteers are often constrained by other work commitments.

Evidence for these claims can be found upon further examination of the three items within the MLQ that constitute extra effort. Question 42 asked coordinators to estimate the frequency with which they may 'heighten other's desire to succeed', revealing a high mean score of 3.14. The regularity of leaders increasing "others' willingness to try harder" was measured through item 44 and produced a similar mean score $(M=3.08)$. Interpreting these additional pieces of information, it may be suggested that Wellington sport coordinators appeared to be successful in engendering an eagerness to exert more effort and contribute. However, the practical reflection of such 'eagerness' may be assessed in item 39 - 'I get others to do more than they expected to do' (Item 39, $M=1.74$ ) - when coordinators estimated that it only happened 'Once in a While' or 'Sometimes'. These findings were in line with those of Dwyer et al's study (2013), which found that whilst transformational leadership can positively influence volunteers, it may not increase their contributed effort. 
Furthermore, a chi-squared value of $\chi^{2}=4.57, p=0.033$ and Fisher's exact score $(P=0.049)$ provided evidence of statistical significance $(p<0.05)$ in the association between measures of contingent reward behaviours and measures of the induced efforts of followers. These findings mirrored those of Judge and Piccolo's (2004), specifying that contingent reward behaviours significantly predict extra effort in followers. Interestingly, Macdonald's (2012) study of Massachusetts high school athletic directors' leadership styles and coaches' outcomes likewise reported that only this transactional behaviour was statistically significant in explaining extra effort.

At first glance, a positive correlation between contingent reward and extra effort may appear counter-intuitive in this setting. At the turn of the millennium, Marshall and Hardman (2000) warned of the apparent decline in school sport and PE in the climate of administrators facing struggles in terms of status, importance and financial resources. These problems have been characteristic of New Zealand schools (Ross \& Cowley, 1995) for two decades, and are still relevant today (Adamson \& Handcock, 2013; Pope, 2011).

The comments of interviewees drew attention to related difficulties in Wellington secondary school sport in terms of growing dissonance and expressed cynicism about the importance of sport. Coordinators believe the traditional curriculum is still perceived to be more valuable than sporting provision, often meaning they have to operate with limited budgets, as:

SC1: "sport is self-funded. Oh I'd like more [laughs], definitely would like more! There's so much more we could be doing and providing for the students if we had more funding".

In terms of coaching personnel, the findings were congruent with the NZ literature (SPARC, 2011; Sport NZ, 2008) in stating that most coaches operated on a voluntary basis and did not receive paid employment. This finding might also relate to why constraints exist 
on the resourcing of coach support and the successful completion of tasks by coaches (Bass, 1997). Thus, the tangible and explicit exchanges inherent within contingent reward behaviours may not be applicable to this context, as the majority of coaches were not contracted in paid roles. The materialistic reinforcement behaviours of contingent reward, therefore, may not explain why coaches' are willing to exert greater efforts.

The relationship between contingent reward and extra effort may, however, be explained by sport coordinators symbolically reinforcing coaches' efforts through praise and recognition. Brymer and Gray (2006) point to the usefulness of the contingent reward dimension of verbal praise in task-based requirements - for instance, and in particular, the coaching of sport. Sport coordinator 2 highlighted the importance of rewarding coaches as:

SC2: "the best people give up so many hours, so unless you do give them a lot back, they won't stick around; the best coaches are the ones that stick around".

By symbolically rewarding and acknowledging coaches contributions, sport coordinators may satisfy the volunteers' needs for recognition and feed their motivations (Catano et al., 2001; Cuskelly et al., 2006), such as the desire to express humanitarian values (Dwyer et al., 2013) or SPARC's volunteer group of 'Aspirers' who “need to feel important, recognised and appreciated" (2006, p. 19). The behavioural significance embedded in these findings may provide meaning to the statistical significance of statistical correlation. As a consequence, the findings perhaps reveal why the implicit and sometimes intangible aspect, that is, the symbolic reinforcement which constitutes part of contingent reward behaviour, will often offer be of greater practical value, when the ability to provide extrinsic motivation is limited by constrained financial resources in schools. 


\section{Key Findings and Conclusions}

Data collected from the Multifactor Leadership Questionnaire (5X Short-Form) was used to provide operational measures and describe the leadership profiles of Wellington secondary school sport coordinators. In addition to determining how frequently coordinators display the behaviours of the nine-factor Full Range Model of Leadership, the MLQ also provided scores for three organisational outcome measures. Analysis of these scores revealed the impact that sport coordinators had on coaches' satisfaction, the extra effort of collaborators and volunteers, and perceived leader effectiveness. Sport coordinators were characterised as exhibiting behaviours in all five transformational leadership dimensions as well contingent reward behaviours. Moreover, correlational analyses revealed that these behaviours were associated with, and might explain and predict coaches' satisfaction and perceived effectiveness of the leader.

Although results appear stark in describing sport coordinator leadership styles along with effects these behaviours have on organisational outcomes, conclusions must be reserved due to methodological limitations. It is important to understate the findings, as reported frequencies may have been prone to self-rater bias (Avolio, Yammarino \& Bass, 1991; Yukl, 1989). Moreover, concluding cause and effect between leadership styles and organisational effectiveness measures must be tempered due to the small sample size $(N=26)$ and low Cronbach Alpha's $(\alpha>0.7)$ of MLQ items. The weaknesses of this approach suggest questions may not reliably measure the constructs and that findings cannot be generalised across New Zealand as a whole. Therefore, in order to overcome the restrictive validity and reliability of this study along with the limitations of the MLQ, a sequential explanatory design was used to reinforce and explain statistical results.

Findings were successful in complementing and further explaining the quantitative results. Interview responses replicated the quantitative results and provided behavioural 
significance to transformational and contingent reward leadership. All interviewees spoke confidently about the value of transformational behaviours in secondary school sport, summarized by Sport coordinator 2 stating "You can't do anything without it!" Moreover, sport coordinators claimed that both transformational and contingent reward behaviours will positively affect coaches' satisfaction, extra effort and perceived leader effectiveness.

The quantitative side of this study explored leadership profiles and its relation organisational effectiveness and can be viewed to have largely followed the process perspective (Cameron, 1978). According to this approach, "a sport organization [sic] may be considered effective if its internal processes and general functioning are smooth and efficient in converting inputs into outputs" (Soucie, 1994, p. 3). Taken alone, the results from the quantitative aspect of this study only provide a narrow understanding of the effects of sport coordinators leadership behaviours on their coaches.

The weakness of employing a singular approach to measuring organisational effectiveness led to scholars recommending an adoption of the multiple constituency approach when exploring sport organisations (Soucie, 1994; Weese, 1994). The additional benefit of conducting interviews was to go beyond the MLQ and assess how their leadership practices are viewed to affect various other constituents in the school sport programmes. Sport coordinators perceived their leadership practices to positively affect outcomes with a number of stakeholders. For instance, Sport coordinator 1 gained support from the board through their visionary goals for sport; Sport coordinator 3 referred to themself as a mentor for students, which increased their satisfaction and participation in sport; and Sport coordinators 2 and 4 were influential in convincing teachers to contribute through volunteering.

\section{Significance and relevance of leadership in Wellington secondary school sport}

According to Soucie (1994), leadership is perhaps the most vital skill that sport coordinators should possess, given that they are the causal agents for effective school sport 
programmes. Findings indicate the potential efficacy that leadership behaviours can have on a variety of organisational outcomes as diverse as coaches' job satisfaction, extra effort of collaborators and subordinates, perceived leader effectiveness etc. In comparison to literature across related fields, the transformational leadership framework appears to be a meaningful and effective style for Wellington secondary school sport coordinators.

The existence of self-reported leadership characteristics and the significant correlations that these transformational and contingent reward leadership characteristics have with desired organisational outcomes were recognised as evidence of existing positive behaviours within the secondary school environment. This is especially so, since the 'New Leadership' paradigm promotes transformational leadership as a necessary style for the $21^{\text {st }}$ century organisation (Avolio \& Bass, 2003). As an additional indirect endorsement of existing leadership behaviours, it may be also be noted that other sport management researchers have also claimed that transformational leadership to be a most desirable and effective style for administrators and leaders (Chelladurai, 2007; Gilbertson et al., 2000; Slack \& Parent, 2006; Soucie, 1994; Weese, 1994). Chelladurai (2001) has even stated that extraordinary leadership is necessary as sport managers are facing increasing pressures, including becoming more business-like in their practices. Furthermore, and in order to cope with the unstable and competitive environments in which they operate, Soucie (1994) has asserted that leaders are required to empower and motivate subordinates 'beyond expectations'.

In a different context, Balyer (2012) and Parry and Proctor-Thomson (2003) suggest that educational leaders must also be able to cope with a rapidly changing and turbulent environment through the adoption of private sector practices and leadership styles. Several scholars have explored educational leadership practices, concluding that transformational leadership has not only been promoted, but also found to have efficacy in stimulating 
adaptability and change within schools (Bass, 2000; Leithwood \& Jantzi, 1999, 2005;

Stewart, 2006). More particular to this present study, academic researchers have also investigated school settings, seeking to gain a deeper understanding of how leadership operates in its sport departments (Armstrong, 1992; Doherty, 1996; Doherty \& Danylchuk, 1997). The need to gain a greater understanding of leadership in school sport has also been pursued by researchers such as Day (2013) and MacDonald (2013), who found positive effects from transformational leadership of athletic directors.

Lastly, scholars have explored transformational theory within New Zealand organisations to assess the models' relevance and effects. Congruent with international contexts, research suggests that transformational leadership is highly preferred (Singer, 1985; Singer \& Singer, 1986) and prevalent (Bass, 1985; Bass \& Riggio, 2006) throughout New Zealand organisations. More importantly Bass's (1985) study on New Zealand company managers employed the MLQ self-rater version, concluded that transformational and contingent reward behaviours exhibited significant statistical relationships with several organisational effectiveness measures. Gilbertson et al. (2000) similarly concluded that a transformational leadership style were appropriate for leaders within New Zealand sport and recreation organisations.

\section{Practical implications}

The rationale for exploring transformational leadership and organisational effectiveness in Wellington secondary school sport coordinators comes from a thrust in contemporary literature emphasizing the necessity for shared leadership that is capable of empowering subordinates to greater performance (Avolio \& Bass, 1999; Bass, 1997; Bass \& Avolio, 1994; Leithwood \& Jantzi, 2000). Of the numerous theorised approaches to understanding leadership - such as the trait, behavioural and situational paradigms - the transformational leadership framework has been endorsed as the most fitting and effective 
for applicability modern organisations (Bass, 1985; Bass \& Riggio, 2006).

Correspondingly, transformational leadership theory has been "one of the current and most popular approaches to leadership that has been the focus of much research since the early 1980s" (Northouse, 2012, p. 171).

In the fields of sport and educational management, the transformational leadership framework has been deemed to be applicable and useful for erstwhile leaders (Leithwood \& Jantzi, 2005; Soucie, 1994; Stewart, 2006). Furthermore, scholars have called for more leaders to encompass transformational roles across all New Zealand organisations (Milne, 2007), and more specifically within the public (Parry \& Proctor-Thomson, 2003) and sporting sectors (Gilbertson et al., 2000). In spite of such endorsement, there has been a lack of systematic research in this domain, which this study has attempted to address within the secondary school sport sector (Riggio, Bass \& Smith Orr, 2004; Pope, 2002). This study thus presents an opportunity to explore how leadership operates in a relatively under-researched context, whilst also providing a base for further studies in additional New Zealand regions, school types, and sport managers in general.

Overall, the findings of this study may offer practical implications for current and future sport coordinators in the wider Wellington region. In keeping with the findings of North American studies of school athletic directors (Doherty, 1996; MacDonald, 2012), this study finds that a transformational leadership style and contingent reward behaviour appear to be most effective for eliciting higher levels of organisational performance and effectiveness in secondary school sports programmes. The study concludes that it is important for sport coordinators to recognise that both transformational and transactional behaviours can provide the foundation for effective leadership in their coordinator roles; and also that it is valuable to be able to adjust leadership style according to the situational contingencies. 
The current study has sought to identify individual leadership practices and to identify patterns of practice of Wellington secondary school sport coordinators. As such, the findings may be of use to those organisations having responsibility for sport including Sport New Zealand, the Ministry of Education and the New Zealand Secondary School Sport Council. These organisations can in turn help inform practice, especially those existing and prospective leadership practices exhibited by secondary school sport coordinators and fellow leaders.

The managers of school sport departments, given a more informed understanding of leadership practices and outcomes, might also consider provision of leadership development programmes to help sport coordinators fulfil more transformational roles. Bass (1985) had claimed that his theory had superseded previous theorising by suggesting that transformational behaviours are not innate, instead they can be taught and developed. Leadership development programmes have reinforced Bass' theoretical stance (Kets de Vries et al., 2009; Mason, 2014; Zenger, 2013), providing further demonstration that even NZ sport coordinators can learn to adopt and improve their transformational behaviours to better fulfil transformational roles. The potential for leadership training is implicit in recent statistics from the New Zealand Secondary Schools Sports Council (2013), which indicate that almost nine in ten $(88 \%)$ sport coordinators already have access to professional development opportunities. Participant responses in this study show that coordinators would welcome such courses, as illustrated by the following comments:

SC1: "I think it would be really beneficial "cause not everyone comes in with that sort of background", while Sport coordinator 2 believed

SC2: "I'm sure people out there are crying out for it".

It may be concluded that in response, future courses could focus on the identification, development, and retention of transformational behaviours. 


\section{Future Research}

The main limitation of this research comes from the small and specific sample size. As the study sought participation from Wellington secondary school sport coordinators, these findings cannot be applied to New Zealand secondary school sport coordinators as a whole. Despite receiving a pleasing response rate $(N=26,56 \%)$, the researcher cannot conclude that the leadership profiles or their relations with organisational effectiveness measures are generalizable across all of New Zealand secondary schools, as no information is presented about the remaining $90 \%$ of schools from the country's other regions. However, future research can build on this preliminary study by seeking a larger cross-sectional sample, more representative of the 238 New Zealand secondary schools (Ministry of Education, 2014). It would be interesting to determine whether findings would be replicated across the nation or whether they were exclusive to Wellington schools.

The possibility that the MLQ Self-Rater 5X Short-Form self-administered and selfreported instrument may have distorted or exaggerated leadership characteristics and organisational effectiveness scores warrants further complementary research. Self-reported surveys have been formerly shown to generate distorted findings as respondents wish to convey themselves in a more favourable light (Crampton \& Wagner, 1994; Podsakoff et al., 2003). The MLQ survey instrument is also prone to what are known as percept-percept biases (Avolio et al., 1991; Yuk1, 1989) meaning that future research would likely benefit from further insight into leadership practices from different perspectives.

This study is limited in that it only explores the perceptions of sport coordinators through the MLQ and semi-structured interviews. Prospective studies could administer the survey to other New Zealand sport coordinators in conjunction with a multitude of constituents such as their followers and superiors. It would be beneficial to employ both raterand self-rater versions of the MLQ for more accurate, precise and reliable measures of 
coordinator leadership styles. Additionally, the range of qualitative data collection methods considered might incorporate some of the following: (a) participant observation to assess the leadership behaviours of coordinators in action; (b) interviews with subordinates and/or other stakeholders to effect comparisons with perceptions of self-reports; or (c) focus group interviews with a variety of sport coordinators to reveal commonalities and allow participants to further explain fellow coordinators responses.

Another interesting domain to explore further would be the demographic characteristics of sport coordinators. Forthcoming research with a larger cross-sectional representative sample might look at differences in respondents' age, gender, experience and longevity in the role. Prior literature has suggested that such variables can predict leadership tendencies and also mediate their effects (Avolio, 1999; Bass, 1999; Doherty, 1997; Eagly et al., 2003; Martin, 2015). Likewise, the demographic and other characteristics of schools possibly influence the suitability, applicability and usefulness of leadership behaviours to affect organisational outcomes. For instance, analysis of school decile categorisation, structure, location etc. might reveal specific communalities and differences in characteristics, relationships and outcomes.

It may also be possible to examine the relationship between leadership behaviours with other criterion variables in the sport setting. As the MLQ is primarily designed to measure job satisfaction, extra effort, and perceived leader effectiveness, a different or modified instrument may be useful for exploring diverse factors encompassed, for example, within the Organizational Commitment Scale (Meyer \& Allen, 1997). The relationship between transformational leadership and commitment has been previously explored in a number of studies (Atmojo, 2012; Lok \& Crawford, 2004; Tyssen et al., 2013) and has been shown to increase commitment of coaches in school sport programmes (Kent \& Chelladurai, 2000). Some of the findings from this qualitative study might be interpreted as suggesting that 
commitment may actually be a better indicator of organisational effectiveness in New Zealand school sport. Specifically, sports coordinators believed that the contributions of volunteers are vital for provision of sport in their school environments, and that without their commitment many sports would cease to be provided for.

\section{Conclusion}

In conclusion, the researcher understood that this study was carried out to provide an indicative and illustrative account of a previously under-explored context. The objectives of this research were not intended to determine the generalizability of cause and effect relationships involving leadership behaviours, rather, the aim was to shed light on an otherwise under-researched managerial role in a secondary school setting. Additionally, the study purported to reinforce findings and existing frameworks from existing literature, and establish which may be applicable to the New Zealand secondary school sport sector.

In this respect, it is noted that the leadership behaviours of Wellington secondary school sport coordinators were congruent with the findings and theorising arising from prior research, for example, where participants who characterised themselves to be transformational leaders also utilised contingent reward behaviours to motivate others to greater levels of effort. Similarly, several empirical studies relating to sport, school and inter-university athletic managers, such as Doherty and Danylchuk's (1997) study of American athletic directors, have suggested that individuals filling roles such as sports coordinators may be able to significantly affect a number of positive organisational outcomes leading to greater quantity and quality of sporting provision. Likewise, this study concludes that the New Leadership' style may also be most pertinent and effectual in this particular setting.

The significance of this present study has shown that sport coordinators are required to be influential and inspirational in their roles. The ability to formulate, communicate and implement a favourable vision for future school sporting practices often hinges on the 
coordinators' leadership behaviours to attract and retain volunteers. Accordingly, the transformational leadership model appears to offers greatest utility in this setting for sustained quality of provision. The exploratory nature of this research has provided a foundation for future research to be enacted that may further contribute to our understanding of the effectiveness of transformational leadership in New Zealand sport. 


\section{REFERENCES}

Adamson, T. C., \& Handcock, P. (2014). Resistance training in New Zealand secondary schools: A survey of the practices and perceptions. University of Otago.

Aminuddin, Y. (1998). The relationship between transformational leadership behaviors of athletic directors and coaches' job satisfaction. Physical Educator, 55(4), 170-175.

Antonakis, J., Avolio, B. J., \& Sivasubramaniam, N. (2003). Context and leadership: An examination of the nine-factor full-range leadership theory using the Multifactor Leadership Questionnaire. The Leadership Quarterly, 14(3), 261-295.

Armstrong, S. (1992). A study of transformational leadership of athletic directors and head coaches in selected NCAA Division III colleges and universities in the Midwest. (Doctoral Dissertation).

Arzi, S., \& Farahbod, L. (2014). Relationship of transformational and transactional leadership style with job satisfaction. Interdisciplinary Journal of Contemporary Research in Business, 6(3), 187-204.

Atmojo, M. (2012). The influence of transformational leadership on job satisfaction, organizational commitment, and employee performance. International Research Journal of Business Studies, 5(2), 113-128.

Avoilio, B. J., Bass, B, M., \& Jung, D. I. (1999). Re-examining the components of transformational and transactional leadership using the Multifactor Leadership Questionnaire. Journal of Occupational and Organizational Psychology, 72(1), 441462. 
Avolio, B. J., Yammarino, F. J., \& Bass, B. M. (1991). Identifying common methods variance with data collected from a single source: An unresolved sticky issue. Journal of Management, 17(3), 571-587.

Avolio, B. J. (1999). Full leadership development: Building the vital forces in organizations. Thousand Oaks, CA: Sage.

Avolio, B. J., \& Bass, B. M. (2004). Multifactor leadership questionnaire, manual and sampler. Redwood City, CA: Mind Garden.

Balyer, A. (2012). Transformational leadership behaviours of school principals: A qualitative research based on teachers' perceptions. International Online Journal of Educational Sciences, 4(3), 581-591.

Barling, J., Weber, T., \& Kelloway, E. K. (1996). Effects of transformational leadership training on attitudinal and financial outcomes: A field experiment. Journal of Applied Psychology, 81(6), 827-832.

Barriball, K. L., \& While, A. (1994). Collecting data using a semi-structured interview: A discussion paper. Journal of Advanced Nursing, 19, 328-335.

Bass, B. M. (1985). Leadership and performance beyond expectations. New York: Free Press.

Bass, B. M., Waldman, D. A., Avolio, B. J., \& Bebb, M. (1987). Transformational leadership and the falling dominoes effect. Group and Organization Management, 12(1), 73-87.

Bass, B. M., \& Stogdill, R. M. . (1990). Handbook of leadership: Theory, research \& managerial applications. London: Free Press.

Bass, B. M. (1991). From transactional to transformational leadership: Learning to share the vision. Organizational Dynamics, 18(3), 19-31. 
Bass, B. M., \& Avolio, B. J. (1994). Improving organizational effectiveness through transformational leadership. Thousand Oaks, CA: Sage Publications.

Bass, B. M., \& Avolio, B. J. (1995). MLQ multifactor leadership questionnaire. Redwood City, CA: Mind Garden.

Bass, B. M., Avolio, B. J., \& Atwater, L. (1996). The transformational and transactional leadership of men and women. Applied Psychology: An International Review, 45(1), 5-34.

Bass, B. M. (1997). Does the transactional-transformational leadership paradigm transcend organisational and national boundaries. American Psychologist, 52(2), 130-139.

Bass, B. M., \& Avolio, B. J. (1997). Full range of leadership: Manual of the multifactor leadership questionnaire. Palo Alto, CA: Mind Garden.

Bass, B. M. (1998). Transformational leadership: Industry, military, and educational impact. Mahwah, NJ: Lawrence Erlbaum Associates.

Bass, B. M. (1999). Two decades of research and development in transformational leadership. European Journal of Work and Organizational Psychology, 8(1), 9-32.

Bass, B. M. (2000). The future of leadership in learning organizations. The Journal of Leadership Studies, 7(5), 18-40.

Bass, B. M., Avolio, B. J., Jung, D. I., \& Berson, Y. (2003). Predicting unit performance by assessing transformational and transactional leadership. Journal of Applied Psychology, 88(2), 207-218. doi: 10.1037/0021-9010.88.2.207

Bass, B. M., \& Riggio, R. E. (2006). Transformational leadership: Psychology Press.

Bennis, W. G. (1959). Leadership theory and administrative behaviour: The problem of authority. Administrative Science Quarterly, 4(3), 259-301. 
Bennis, W. G. (1975). Leadership book.

Bennis, W. G. (1984). The Four Competencies of Leadership.

Bennis, W. G., \& Nanus. B. . (1985). Leaders: The strategies for taking charge. New York: Harper \& Row.

Bennis, W. G. (2001). Leading in unnerving times. MIT Sloan Management Review, 42(2), 97-103.

Boerner, S., Eisenbeiss, S. A., \& Griesser, D. (2007). Follower behaviour and organizational performance: The impact of transformational leaders. Journal of Leadership \& Organizational Studies, 13, 15-26.

Bryman, A. (1992). Charisma \& leadership in organizations. Newbury Park, CA: Sage Publications.

Bryman, A., Stephens, M., \& Campo, C. (1996). The importance of context: Qualitative reserach and the study of leadership. Leadership Quarterly, 7(3), 353-370.

Bryman, A. (2004). Qualitative research on leadership: A critical but appreciative review. The Leadership Quarterly, 15(6), 729-769. doi: 10.1016/j.leaqua.2004.09.007

Brymer, E., \& Gray, T. (2006). Effective leadership: Transformational or transactional? Australian Journal of Outdoor Education, 10(2), 13-19.

Burns, J. M. (1978). Leadership. New York: Harper \& Row Publications.

Burton, L. J., \& Peachey, J. W. . (2009). Transactional or transformational? Leadership preferences in division III athletic administrators. Journal of Intercollegiate Sport, 2, 245-259. 
Cameron, K. (1978). Measuring organizational effectiveness in institutions of higher education. Administrative Science Quarterly, 23, 604-632.

Catano, V. M., Pond, M., \& Kevin Kelloway, E. (2001). Exploring commitment and leadership in volunteer organizations. Leadership and Organization Development Journal, 22(6), 256-263.

Cavana, R. Y., Delahaye, B. L., \& Sekaran, U. (2001). Applied business research: Qualitative and quantiative methods: John Wiley \& Sons Australia.

Chelladurai, P., \& Saleh, S. D. (1980). Dimensions of leader behaviour in Sports: Development of a leadership scale. Journal of Sport Psychology, 2, 34-45.

Chelladurai, P. (2007). Leadership in Sports. The Ohio State University.

Choi, J. H. (2006). The relationship among transformational leadeership, organizational outcomes, and service quality in the five major NCAA conferences. (Doctoral Dissertation), Texas A\&M University.

Coakes, S. J., \& Steed, L. (2009). SPSS: Analysis without anguish using SPSS version 14.0 for Windows: John Wiley \& Sons, Inc.

Cohen, L., Manion, L., \& Morrison, K. (2000). Research methods in education (6th ed.). Abingdon, OX: Routledge.

Committee on Sports Medicine, \& Committee on School Health. (1987). Physical fitness and the schools. Pediatrics, 80(3), 449-450.

Conger, J. A., \& Kanungo, R. N. (1987). Toward a behavioural theory of charismatic leadership in organizational settings. The Academy of Management Review, 12(4), 637-647. 
Copland, M. A. (2003). Leadership of inquiry: Building and sustaining capacity for school improvement. Educational Evaluation and Policy Analysis, 25(4), 375-395.

Crampton, S. N., \& Wagner, J. A. . (1994). Percept-percept inflation in microorganizational research: An investigation of prevalence and effect. Journal of Applied Psychology, 79(1), 67-76.

Creswell, J. W. (2002). Educational research: Planning, conducting and evaluating quantitative: Prentice Hall.

Crust, L., \& Lawrence, I. (2006). A review of leadership in sport: Implications for football management. Athletic Insight: The Online Journal of Sport Psychology, 8(4), 28-48.

Cuskelly, G. (2004). Volunteer retention in community sport organisations. European sport management quarterly, 4(2), 59-76.

Cuskelly, G., Taylor, T., Hoye, R., \& Darcy, S. (2006). Volunteer Management Practices and Volunteer Retention: A Human Resource Management Approach. Sport Management Review, 9(2), 141-163. doi: 10.1016/s1441-3523(06)70023-7

Darvish, H., \& Shirazi Pour, M. (2013). Measuring employees job satisfaction as outcome of multifactoral leadership questionnaire (MLQ) leadership styles: An empirical study. Reef Resources Assessment and Management Technical Paper, 38(2), 622-627.

Davis, T. R. V., \& Luthans, F. (1979). Leadership reexamined: A behavioural approach. Academy of Management, 4(2), 237-248.

Day, D. V. (2001). Leadership development. A review in context. Leadership Quarterly, 11(4), 581-613.

Day, D. V. (2013). Athletic director leadership and success in NCAA Division III athletic departments. (Master of Arts), University of North Carolina. 
de Villiers, R. (2014). Book essay on "The Dark Side of Transformational Leadership: A Critical Perspective". Journal of Business Research, 67(12), 2512-2514. doi: 10.1016/j.jbusres.2014.01.006

DeGroot, T., Kiker, D. S., \& Cross, T. C. (2000). A meta-analysis to review organizational outcomes related to charismatic leadership. Canadian Journal of Administrative Sciences, 17(4), 356-371.

Den Hartog, D. N., Van Muijen, J. J., \& Koopman, P. L. (1997). Transactional versus transformational leadership: An analysis of the MLQ. Journal of Occupational and Organizational Psychology, 70(1), 19-34.

Den Hartog, D. N., House, R. J., Hanges, P. J., Ruiz-Quintanilla, S. A., \& Dorfman, P. W. (1999). Culture specific and cross-culturally generalizable implicit leadership theories: Are attributes of charismatic/transformational leadership universally endorsed? The Leadership Quarterly, 10(2), 219-256.

DeRue, D. S., Nahrgang, J. D., Wellman, D., \& Humphrey, S. E. (2011). Trait and behavioural theories of leadership: An integration and meta-analytic test of their relative validity. Personnel Psychology, 64(1), 7-52.

Dillman, D. A. (1991). The design and administration of mail surveys. Annual Reviews Sociology, 17, 225-249.

Doherty, A. J., \& Danylchuk, K. E. (1996). Transformational and transactional leadership in interuniversity athletics management. Journal of Sport Management, 10(3), 292-309.

Doherty, A. J. (1997). The effect of leader characteristics on the perceived transformational/transactional leadership and impact of interuniviersity athletic administrators. Journal of Sport Management, 11, 275-285. 
Doherty, A. J. (1998). Managing Our Human Resources: A Review of Organisational Behaviour in Sport. Sport Management Review, 1(1), 1-24. doi: 10.1016/s14413523(98)70097-x

Dwyer, P. C., Bono, J. E., Snyder, M., Nov, O., Berson, Y. (2013). Sources of Volunteer Motivation: Transformational Leadership and Personal Motives Influence Volunteer Outcomes. Nonprofit Management and Leadership, 24(2), 181-205. doi: $10.1002 / \mathrm{nml} .21084$

Eagly, A. H., Johannesen-Schmidt, M. C., van Engen, M. L. (2003). Transformational, transactional, and laissez-faire leadership styles: A meta-analysis comparing women and men. Psychological Bulletin, 129(4), 569-591. doi: 10.1037/0033-2909.129.4.569

Education, Ministry of. (2014). Schools directory. Retrieved March, 23, 2014, from http://www.educationcounts.govt.nz/directories/list-of-nz-schools

Fuller, J. B., Patterson, C. E., Hester, K., \& Stringer, D. Y. . (1996). A quantitative review of research on charismatic leadership. Psychological Reports, 78(1), 271-287.

Galton, F. (1869). Hereditary Genius: MacMillan and Company.

Geijel, F., Sleegers, P., Leithwood, K., \& Jantzi, D. . (2003). Transformational leadership effects on teachers' commitment and effort toward school reform. Journal of Educational Administration, 41(3), 228-256.

Gilbertson, D. W. (2000). Transformational leadership in Australasian sports organisations.: New Zealand Innovation and Competitiveness Project Victoria University of Wellington.

Golafshani, N. (2003). Understanding reliability and validity in qualitative research. The Qualitative Report, 8(4), 597-607. 
Goodwin, V. L., Wofford, J. C., \& Whittington, J. L. . (2001). A theoretical and empirical extension of the transformational leadership construct. Journal of Organizational Behaviour, 22(7), 759-774.

Gratton, C., \& Jones, I. (2004). Research methods for sports studies. London: Routledge.

Gundersen, G., Hellesoy, B. T., \& Raeder, S. (2012). Leading international project teams: The effectiveness of transformational leadership in dynamic work environments. Journal of Leadership \& Organizational Studies, 19(1), 46-57. doi:

$10.1177 / 1548051811429573$

Hallinger, P. (2003). Leading educational change: Reflections on the practice of instructional and transformational leadership. Cambridge Journal of Education, 33(3), 329-352. doi: $10.1080 / 0305764032000122005$

Hanson, W. E., Creswell, J. W., Plano Clark, V. L., Petska, K. S., \& Creswell, J. D. (2005). Mixed methods research designs in psychology. Journal of Counseling Psychology, 52(2), 224-235.

Hersey, P., Blanchard, K. H., \& Johnson, D. E. (1988). Management of organizational behavior (Vol. 9).

House, R. J. (1977). A 1976 theory of charismatic leadership. Faculty of Management Studies, University of Toronto.

Hoye, R. (2006). Leadership within Australian voluntary sport organization boards. Nonprofit Management and Leadership, 16(3), 297-313. doi: 10.1002/nml.108

Hsu, C. H. (2002). A structural equation modeling analysis of transformational leadership, organizational culture and organizational effectiveness in Taiwanese sport/fitness organizations. (Doctoral Dissertation). 
Hsu, C. H., Bell, R. C., \& Cheng, K. (2002). Transformational leadership and organizational effectiveness in recreational sports/fitness programs. The Sport Journal, $5(1), 1-5$.

Imran, R., Zahoor, F., \& Zaheer, A. (2012). Leadership and performance relationship: Culture matters. International Journal of Innovation, Management and Technology, 3(6), 713717. doi: 10.7763/ijimt.2012.v3.325

Jandaghi, G., Matin, H. Z., \& Farjami, A. . (2009). Comparing transformational leadership in successful and unsuccessful companies. African Journal of Business Management, $3(7), 272-280$.

Jaskyte, K. (2004). Transformational leadership, organisational culture, and innovativeness in nonprofit organisations. Nonprofit Management \& Leadership, 15(2), 153-168.

Jenewein, W., \& Schmitz, C. (2007). Creating a high performance team through transformational leadership:The case of Alinghi. Business Case Journal, 15(1), 26-53.

Judge, T. A., Bono, J. E., Ilies, R., \& Gerhardt, M. W. (2002). Personality and leadership: A qualitative and quantitative review. Journal of Applied Psychology, 87(4), 765-780. doi: $10.1037 / / 0021-9010.87 .4 .765$

Judge, T. A., \& Piccolo, R. F. (2004). Transformational and transactional leadership: A metaanalytic test of their relative validity. Journal of Applied Psychology, 89(5), 755-768. doi: $10.1037 / 0021-9010.89 .5 .755$

Jung, D. I., Chow, C., \& Wu, A. (2003). The role of transformational leadership in enhancing organizational innovation: Hypotheses and some preliminary findings. The Leadership Quarterly, 14(4-5), 525-544. doi: 10.1016/s1048-9843(03)00050-x

Kent, A. . (1995). Organizational effectiveness, executive leadership and organizational 
culture: A study of selected provincial sport organizations.

Kent, A., \& Challadurai, P. (2001). Perceived transformational leadership, organizational commitment, and citizenship behavior: A case study in intercollegiate athletics. Journal of Sport Management, 15(2), 135-159.

Kets de Vries, M., Hellwig, T., Vrignaud, P., Guillen Ramo, L., Florent-Treacy, E., \& Korotov, K. (2009). Sustainable effectiveness of a transformational leadership development program: An exploratory study. INSEAD Business School Research Paper.

Kirkpatrick, S. A., \& Locke, E. A. (1991). Leadership: Do traits matter? The Executive, 5(2), $48-60$.

Kotter, J. P. (1982). What effective general managers really do. Harvard Business Review.

Kuchler, W. A. (2001). The association of perceived leadership behaviours with subordinate job satisfaction at selected NCAA Division III Midwest collegiate athletic departments. (Doctoral Dissertation).

Leithwood, K. A., \& Poplin, M. S. (1992). The move toward transformational leadership. Educational Leadership, 49(5), 8-12.

Leithwood, K. A. . (1994). Leadership for school restructuring. Educational Administration Quarterly, 30(4), 498-518.

Leithwood, K. A., Jantzi, D., \& Steinbach, R. . (1999). Changing leadership for changing times: McGraw-Hill International.

Leithwood, K. A., \& Jantzi, D. (1999). The effects of transformational leadership on organizational conditions and student engagement with school. Journal of Educational Administration, 38(2), 112-129. 
Leithwood, K. A., \& Jantzi, D. (2005). A review of transformational school leadership research 1996-2005. Leadership and Policy in Schools, 4(3), 177-199. doi: $10.1080 / 15700760500244769$

Leong, L. Y. C., \& Fischer, R. (2010). Is Transformational Leadership Universal? A MetaAnalytical Investigation of Multifactor Leadership Questionnaire Means Across Cultures. Journal of Leadership \& Organizational Studies, 18(2), 164-174. doi: $10.1177 / 1548051810385003$

Linam, K. R. (1999). Leadership styles of collegiate athletic directors and head coaches' satisfaction. (Doctoral Dissertation).

Locke, E. A. (1976). The nature and causes of job satisfaction. Handbook of industrial and organizational psychology, 1, 1297-1343.

Lok, P., \& Crawford, J. (2002). Antecedents of organizational commitment and the mediating role of job satisfaction. Journal of Managerial Psychology, 16(8), 594-613.

Lok, P., \& Crawford, J. (2004). The effect of organisational culture and leadership style on job satisfaction and organisational commitment. Journal of Management Development, 23(4), 321-338. doi: 10.1108/02621710410529785

London, C. L. (1996). Transformational, transactional leadesrhip and organizational effectiveness of Canadian University athletic programs. (Doctoral Dissertation).

Lowe, K. B., Kroeck, K. G., \& Sivasubramaniam, N. (1996). Effectiveness correlates of transformational and transactional leadership: A meta-analytic review of the MLQ literature. The Leadership Quarterly, 7(3), 385-425. doi: 10.1016/s10489843(96)90027-2 
MacDonald, J. H. (2012). A quantitative examination of leadership styles of athletic directors and coaches' leadership outcomes in Massachusetts.

Mangione, T. W. (1995). Mail surveys: Improving the quality. California, US: Sage Publications Inc.

Manning, L. W. (2012). NCAA Athletic Directors' self-perspective of transformational/transactional leadership. (Doctoral Dissertation).

Marshall, J., \& Hardman, K. (2000). The state and status of physical education in schools in international context. European Physical Education Review, 6(3), 203-229.

Martin, J. (2015). Transformational and transactional leadership: An exploration of gender, experience, and institution type. portal: Libraries and the Academy, 15(2), 331-351. doi: $10.1353 /$ pla.2015.0015

Maslow, A. H. (1954). Motivation and personality. New York: Harper Row.

Mason, C., Griffin, M., Parker, S. (2014). Transformational leadership development. Leadership \& Organization Development Journal, 35(3), 174-194. doi: 10.1108/lodj05-2012-0063

Maykut, P. \& Morehouse, R. (2000). Beginning qualitative research: A philosophic and practical guide. UK: Falmer Press.

McCarley, T. A., Peters, M. L., \& Decman, J. M. (2014). Transformational leadership related to school climate: A multi-level analysis. Educational Management Administration \& Leadership, 1-21. doi: 10.1177/1741143214549966

Meunjohn, N., \& Armstrong, A. (2008). Evaluating the structural validity of the MLQ, capturing the leadership factors of transformational-transctional leadership. 
Contemporary Management Research, 4(1), 3-14.

Meyer, J. P., \& Allen, N. J. (1991). A three-component conceptualization of organizational commitment. Human Resource Management Review, 1(1), 61-89.

Meyer, J. P., \& Allen, N. J. (1997). Commitment in the workplace. Thousand Oaks, CA: Sage Publications.

Miller, D. C., \& Salkind, N. J. (2002). Handbook of research design and social measurement (6th ed.). Thousand Oaks, California: Sage Publications.

Milne, R. (2007). Management and leadership. In L. Gill, \& Pio, E. (Ed.), Organisations and Management (pp. 194-217). Auckland, New Zealand: Pearsons Education New Zealand.

Ministry of Education. (2014). Schools directory. Retrieved March, 2015, from http://www.educationcounts.govt.nz/directories/list-of-nz-schools

Miyashiro, A. D. (2007). A quantitative descriptive correlational analysis of perceived leadership styles of athletic directors and coaches' satisfaction levels.

Muhlberger, M. D., \& Traut-Mattausch, E. (2015). Leading to Effectiveness: Comparing Dyadic Coaching and Group Coaching. The Journal of Applied Behavioral Science, 51(2), 198-230. doi: 10.1177/0021886315574331

New Zealand Secondary Schools Sports Council. (2001). Census of sport participation. Christchurch: New Zealand Secondary School Sports Council.

Northouse, P. G. (2012). Leadership: Theory and Practice: Sage.

O'Neil, D. A., Hopkins, M. M., \& Bilimoria, D. (2015). A Framework for Developing Women Leaders: Applications to Executive Coaching. The Journal of Applied Behavioral Science, 51(2), 253-276. doi: 10.1177/0021886315575550 
Parris, D. L., \& Peachey, J. W. (2012). Building a legacy of volunteers through servant leadership: A cause-related sporting event. Nonprofit Management and Leadership, 23(2), 259-276. doi: 10.1002/nml.21047

Parry, K. W., \& Proctor-Thomson, S. B. (2003). Leadership, culture and performance:The case of the New Zealand public sector. Journal of Change Management, 3(4), 376399.

Parry, K. W., \& Proctor-Thomson, S. B. (n.d.). Testing the validity and reliability of the organisational description questionnaire (ODQ). International Journal of Organisational Behaviour, 4(3), 111-124.

Paton, G. (1987). Sport management research: What progress has been made? Journal of Sport Management, 1(1), 25-31.

Patton, M. Q. (2002). Qualitative research and evaluation methods (3rd ed.). London, UK: Sage Publications.

Peachey, J. W., \& Burton, L. J. (2010). Male or female athletic director? Exploring perceptions of leader effectiveness and a (potential) female leadership sdvantage with intercollegiate athletic directors. Sex Roles, 64(5-6), 416-425. doi: 10.1007/s11199010-9915-y

Piccolo, R. F., Colquitt, J. A. (2006). Transformational leadership and job behaviours: The mediating role of core job characteristics. Academy of Management Journal, 49, 327340.

Plackett, R. L. (1983). Karl Pearson and the chi-squared test. International Statistical Review, 51(1), 59-72. 
Podsakoff, P. M., MacKenzie, S. B., Ahearne, M., \& Bommer, W. H. (1995). Searching for a needle in a haystack: Trying to identify the illusive moderators of leadership behaviors. Journal of Management, 21(3), 422-470.

Podsakoff, P. M., MacKenzie, S. B., \& Bommer, W. H. (1996). Transformational leader behaviors and substitues for leadership as determinants of employee satisfaction, commitment, trust, and organizational citizenship behaviors. Journal of Management, 22(2), 259-298.

Podsakoff, P. M., MacKenzie, S. B., Lee, J. Y., \& Podsakoff, N. P. (2003). Common method biases in behavioral research: A critical review of the literature and recommended remedies. Journal of Applied Psychology, 88(5), 879-903.

Pope, C. C. (2002). Plato makes the team: The arrival of secondary school sport academies. Waikato Journal of Education, 8, 89-100.

Pope, C. C. (2007). Happy 21st birthday sport education: Where are we now?

Pope, C. C. (2011). The physical education and sport interface: Models, maxims and maelstrom. European Physical Education Review, 17(3), 273-285. doi: $10.1177 / 1356336 \times 11416728$

Pope, D. G., \& Pope, J. C. (2009). The impact of college sports succes on quantity and quality of student application. Southern Economic Journal, 75(3), 750-780.

Punch, K. F. (2013). Introduction to social research: Quantitative and qualitative approaches: Sage.

Ramachandran, S., \& Krishnan, V. R. (2009). Effect of transformational leadership on followers' affective and normative commitment: Culture as moderator. Great Lakes 
Herald, 3(1), 23-38.

Riggio, R. E., Bass, B. M., \& Orr, S. S. (2004). Transformational leadership in nonprofit organizations. In R. E. Riggio and S. Smith (Ed.), Improving Leadership in Nonprofit Organizations. San Francisco: Jossey-Bass.

Ristow, A. M., Amos, T. L., \& Staude, G. E. (1999). Transformational leadership and organisational effectiveness in the administration of cricket in South Africa. South African Journal of Business Management, 30(1), 1-5.

Ritchie, J. (2003). The application of qualitative methods to social research. In J. Ritchie \& J. Lewis (Eds.), Qualitative research practice: A guide for social science students (pp. 24-46). London, England: Sage Publications Ltd.

Rizi, R. M., Azadi, A., Farsani, M. E., \& Aroufzad, S. (2013). Relationship between leadership styles and job satisfaction among phyiscal education organizations employees. European Journal of Sports and Exercise Science, 2(1), 7-11.

Ross, J., \& Cowley, V. (1995). Just how much physical education are students getting? Part 1: Junior Levels to Form Two. Journal of Physical Education New Zealand, 28(1), 3-8.

Rouff, K. J. (2007). An examination of selected NCAA Division III coaches' perceptions of athletic directors, are they leaders or are they managers? (Doctoral Dissertation).

Rowold, J., \& Heinitz, K. (2007). Transformational and charismatic leadership: Assessing the convergent, divergent and criterion validity of the MLQ and the CKS. The Leadership Quarterly, 18(2), 121-133. doi: 10.1016/j.leaqua.2007.01.003

Rowold, J., \& Rohmann, A. (2009). Transformational and transactional leadership styles, followers' positive and negative emotions, and performance in German nonprofit orchestras. Nonprofit Management and Leadership, 20(1), 41-59. doi: 
$10.1002 / \mathrm{nml} .240$

Sala, F. (2003). Leadership in education: Effective U.K. college principals. Nonprofit Management \& Leadership, 14(2), 171-189.

Schein, E. H. (1992). Organizational culture and leadership. San Fransisco: Jossey-Bass.

Schmid, H. (2006). Leadership styles and leadership change in human and community service organizations. Nonprofit Management and Leadership, 17(2), 179-194. doi: $10.1002 / \mathrm{nml} .142$

Schnake, M. (1991). Organizational citizenship: A review, proposed model, and research agenda. Human Relations, 44(735-759).

Shamir, B., House, R. J., \& Arthur, M. B. (1993). The motivational effects of charismatic leadership: A self-concept based theory. Organization Science, 4(4), 577-594.

Sharpe, E.K. (2006). Resources at the grassroots of recreation: Organizational capacity and quality of experience in a community sport organization. Leisure Science, 28, 385401.

Sheehan, K. B. . (2001). E-mail survey response rates: A review. Journal of ComputerMediated Communication, 6(2), 0-0.

Shurbagi, A. M. A. (2014). The Relationship between transformational leadership style job satisfaction and the effect of organizational commitment. International Business Research, 7(11), 126-138. doi: 10.5539/ibr.v7n11p126

Singer, M. S. (1985). Transformational vs transactional leadership: A study of New Zealand company managers. Psychological Reports, 57, 143-146.

Singer, M. S., \& Singer, A. E. (1986). Relation between transformational vs transasctional leadership preference and subordinates' personality: An exploratory study. Perceptual 
and Motor Skills, 62, 775-780.

Singer, M. S., \& Singer, A. E. (1990). Situational constraints on transformational versus transactional leadership behavior, subordinates' leadership preference, and satisfaction. The Journal of Social Psychology, 130(3), 385-396.

Slack, T., \& Parent, M. M. (2006). Understanding sport organizations: The application of organizational theory: Human Kinetics.

Snape, D., \& Spencer, L. (2003). The foundations of qualitative research. In J. Ritchie \& J. Lewis (Eds.), Qualitative research practice: A guide for social science students (pp. 2-23). London, England: Sage Publications Ltd.

Soucie, D. (1994). Effective managerial leadership in sport organizations. Journal of Sport Management, 8, 1-13.

Sparkes, A. (1994). Research paradigms in physical education: Some brief comments on differences that make a difference. British Journal of Physical Education Research Supplement, 14, 11-16.

SPARC. (2006). Finding and keeping volunteers: What the research tells us. Wellington, New Zealand: SPARC.

SPARC. (2011). Volunteers. The heart of sport. Wellington, New Zealand: SPARC.

Spector, P. E. (1994). Using self-report questionnaires in OB research: A comment on the use of a controversial method. Journal of Organizational Behaviour, 15, 385-392.

Sport NZ. (2008). New Zealand secondary school sport and recreation coordinators manual. Retrieved from http://www.sportnz.org.nz/en-nz/young-people/Ages_1318_Years/Sport--Recreation-Co-ordinators-/ 
Sport NZ. (2014). Running secondary school sport. Retrieved from

http://www.sportnz.org.nz/assets/Uploads/attachments/managing-sport/youngpeople/RSSS-Full-Guide.pdf

Stanbrough, M. (2010). Developing team leaders: A leadership development program to build effective team leaders. Journal of Coaching Education, 3(2), 102-105.

Stewart, J. (2006). Transformational leadership: An evolving concept examined through the works of Burns, Bass, Avolio, and Leithwood. Canadian Journal of Educational Administration and Policy, 54, 1-29.

Stogdill, R. M. (1974). Handbook of leadership: A survey of literature.

Stothart, B. (2000). Uncomfortable bedfellows: Sport and physical education, a problematic relationship. New Zealand Physical Educator, 33(1), 40.

Survey, Super. (2009). Online survey response rates and time. from www.supersurvey.com

Tafvelin, S., Armelius, K., \& Westerberg, K. (2011). Toward Understanding the Direct and Indirect Effects of Transformational Leadership on Well-Being: A Longitudinal Study. Journal of Leadership \& Organizational Studies, 18(4), 480-492. doi: $10.1177 / 1548051811418342$

Tashakkori, A., \& Teddlie, C. (1998). Mixed methodology: Combining qualitative and quantitative approaches. Thousand Oaks, California: Sage Publications Ltd.

Taylor, C. M. (2014). Transformational leadership in a nonprofit organization: A cast study of a Filipino nonprofit in diverse communities. (Doctoral Dissertation), Temple University.

Tejada, M. J., Scandura, T. A., \& Pillai, R. (2001). The MLQ revisited: Psychometric properties and recommendations. The Leadership Quarterly, 12, 31-52. 
Tepper, B. J., \& Percy, P. M. (1994). Structural validity of the multifactor leadership questionnaire. Educational and Psychological Measurement, 54(3), 734-744.

Tourish, D. (2013). The dark side of transformational leadership: A critical perspective New York: Routledge.

Turner, D. W. (2010). Qualitative interview design: A practical guide for novice investigators. The Qualitative Report Volume, 15(3), 754-760.

Tyssen, A. K., Wald, A., \& Heidenreich, S. (2013). Leadership in the Context of Temporary Organizations: A Study on the Effects of Transactional and Transformational Leadership on Followers' Commitment in Projects. Journal of Leadership \& Organizational Studies, 21(4), 376-393. doi: 10.1177/1548051813502086

Valdiserri, G. A., \& Wilson, J. L. (2010). The study of leadership in small business organizations. Impact of profitability and organizational success. The Entrepreneurial Exectuive, 15, 47-71.

Voon, M. L., Lo, M. C., Ngui, K. S., Ayob, N. B. (2011). The influence of leadership styles on employee's job satisfaction in public sector organizations in Malaysia. International Journal of Business, Management and Social Sciences, 2(1), 24-32.

Wallace, M., \& Weese, W. J. (1995). Leadership, organisational culture, and job satisfaction in Canadian YMCA organisations. Journal of Sport Management, 9, 182-193.

Wang, G., Oh, I. S., Courtright, S. H., \& Colbert, A. E. (2011). Transformational leadership and performance across criteria and levels: A meta-analytic review of 25 years of research. Group and Organization Management, 36(2), 223-270.

Wicker, P., \& Breuer, C. (2011). Scarcity of resources in German non-profit sport clubs. Sport management review, 14(2), 188-201. 
Weese, W. J. (1994). A leadership discussion with Dr. Bernard Bass. Journal of Sport Management, 8, 179-189.

Weese, W. J. (1995). Leadership and organizational culture: An investigation of Big Ten and Mid-American conference campus recreation administrators. Journal of Sport Management, 9, 119-134.

Weese, W. J. (1996). Do leadership and organizational culture really matter? Journal of Sport Management, 10, 197-206.

Weese, W. J. (1997). The development of an instrument to measure effectiveness in campus recreation programs. Journal of Sport Management, 11, 263-274.

Whiting, L. S. (2008). Semi-structured interviews: Guidance for novice researchers. Nursing Standard, 22(23), 35-40.

Wilson, S. (2014). Book Review: The dark side of transformational leadership: A critical perspective. Organization, 22(1), 150-152. doi: 10.1177/1350508413510000

Weber, M., \& Maximilian, K. E. (1947). From Max Weber: Essays in sociology. Translated, edited and with an introduction by HH Gerth and C. Wright Mills. H. H. Gerth (Ed.). Kegan Paul.

Yammarino, F. J., \& Bass, B. M. (1990). Transformational leadership and multiple level of analysis. Human Relations, 43(10), 975-995.

Yukl, G. (1989). Managerial leadership: A review of theory and research. Journal of Management, 15(2), 251-189.

Yukl, G. (2001). Leadership in organizations: Pearson Education India.

Yukl, G., \& Mahsud, R. (2010). Why flexible and adaptive leadership is essential. Consulting Psychology Journal: Practice and Research, 62(2), 81. 
Zaccaro, S. J. (2007). Trait-based perspectives of leadership. American Psychologist, 62(1), 6-15. doi: 10.1037/0003-066X.62.1.6

Zenger, J., Sandholtz, K., \& Folkman, J. . (2013). Developing leaders. Leadership Excellence, $30(8), 20$.

Zhang, J., DeMichele, D. J., Douglas, J., \& Connaughton, D. P. (2004). Job satisfaction among mid-level collegiate campus recreation program administrators. Journal of Sport Behavior, 27(2), 184-212. 


\section{APPENDICES}

\section{Appendix A}

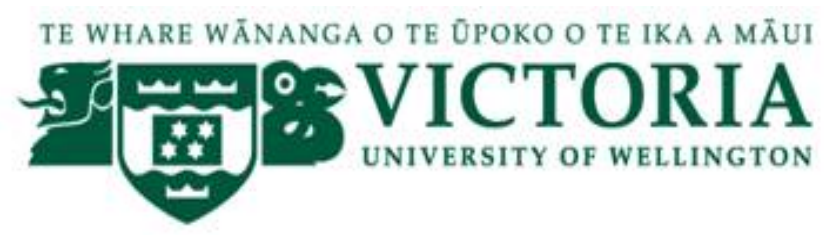

\section{CONSENT FORM FOR PARTICIPANTS}

\section{Researcher: Tom Adamson $\quad$ Supervisor: Professor John Davies}

I have read the Information Sheet concerning this project and understand what it is about. All my questions have been answered to my satisfaction. I understand that I am free to request further information at any stage.

I know that:

1. This research has been approved by Victoria University of Wellington Human Ethics Committee;

2. My participation in the research is entirely voluntary;

3. I am free to withdraw from the research within two weeks after completing the interview. This will not provide any disadvantage;

4. Personal information/raw data [audio-recordings] will be destroyed one year following the completion of the research to avoid the possibility of future unwanted publication. This information will be retained in secure storage until destroyed;

5. This project involves a series of formal and informal questions about my experiences and opinions of my leadership behaviours and subsequent organisational effectiveness as the school's sports coordinator.

This will include pre-determined questions, but the majority of the questions that will be asked have not been determined in advance, but will depend on the way in which the interview develops.

In the event that the line of questioning develops in such a way that I feel hesitant or uncomfortable I may decline to answer any particular question(s) and/or may withdraw from the project without any disadvantage of any kind; and

6. The aggregated and/or anonymised results of the project may be published and will be available in the Victoria University Library but my identity will remain confidential.

7. The semi-structured interviews will be audio-recorded using a voice recorder.

By ticking the following box, I agree to the audio-recording of the interview: I..................................... give consent to be involved in this research.

(Please print name clearly) 


\section{Appendix B}

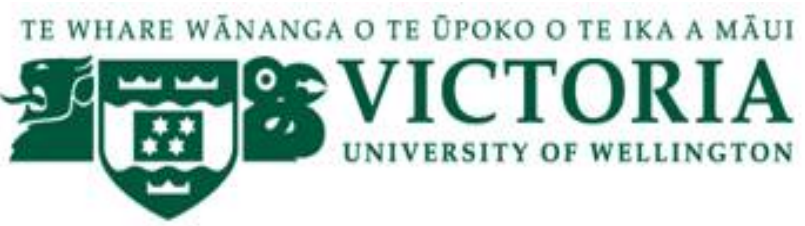

\section{INFORMATION SHEET/LETTER FOR SURVEY PARTICIPANTS}

\section{Research project:}

\section{Transformational Leadership and Organisational Effectiveness in Wellington Secondary School Sport Coordinators}

Date:

To whom it may concern,

My name is Tom Adamson.

I am a Masters student at Victoria University of Wellington, undertaking a research project examining the leadership practices of Wellington Secondary School Sports Coordinators, and how these behaviours impact organisational effectiveness. This research is being conducted as part of the requirements for the completion of my degree and is the basis of my Master's thesis.

This research has been approved by Victoria University of Wellington Human Ethics Committee.

Thank you for showing an interest in this project. Please read this information sheet carefully before deciding whether or not to participate. If you decide to participate, we thank you, again.

If you decide not to take part there will be no disadvantage to you of any kind and we thank you for considering our request.

\section{What is the aim of the project?}

The study is designed to provide information on the nature of transformational leadership behaviours displayed by Wellington Secondary School Sports Coordinators, and how these behaviours contribute to organisational effectiveness (as operationalised by coaches' job satisfaction, extra effort, and perceived leader effectiveness).

The major aims of the study are:

1. Determine the leadership behaviours of Wellington Secondary School Sports Coordinators (transformational, transactional, or laisse-faire).

2. Explore the relationships between each leadership category and perceived organisational effectiveness.

3. Confirming or not the applicability's of previous studies frameworks and findings on transformational leadership and organisational effectiveness to the context of Wellington Secondary School Sport. 


\section{What type of participants are being sought?}

For the Multifactor Leadership Questionnaire (MLQ), we are seeking representative sports coordinators from every Wellington Secondary School (Years 9-13).

Additionally, we are seeking 3-5 coordinators who are willing to give up roughly 30 minutes to an hour of their time to engage in a semi-structued interview at their convenience.

\section{What will participants be asked to do?}

Should you agree to take part in this project, you will be also asked to complete an online survey on your leadership behaviours, and how these relate to perceived organisational effectiveness of your school's sports provision.

The survey will only take a around 10 minutes to complete, comprised of only closed-nature questions.

\section{What if participants have any questions?}

If you have any questions about our project, either now or in the future, please feel free to contact either:

Tom Adamson (Student Researcher)

School of Management

Email: tc.adamson@gmail.com

Phone: 0273040973 or, Professor John Davies

School of Management

email: john.davies@vuw.ac.nz 


\section{Appendix C}

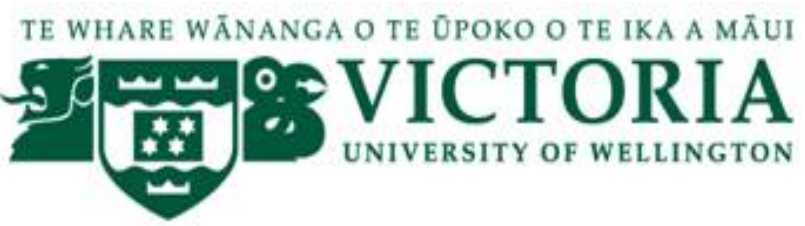

\section{INFORMATION SHEET/LETTER FOR INTERVIEW PARTICIPANTS}

\section{Research project:}

\section{Transformational Leadership and Organisational Effectiveness in Wellington Secondary School Sport Coordinators}

Date:

To whom it may concern,

My name is Tom Adamson.

I am a Masters student at Victoria University of Wellington, undertaking a research project examining the leadership practices of Wellington Secondary School Sports Coordinators, and how these behaviours impact organisational effectiveness. This research is being conducted as part of the requirements for the completion of my degree and is the basis of my Master's thesis.

This research has been approved by Victoria University of Wellington Human Ethics Committee.

Thank you for showing an interest in this project. Please read this information sheet carefully before deciding whether or not to participate. If you decide to participate, we thank you, again.

If you decide not to take part there will be no disadvantage to you of any kind and we thank you for considering our request.

\section{What is the aim of the project?}

The study is designed to provide information on the nature of leadership behaviours (namely transformational, transactional, and laissez-faire styles) displayed by Wellington Secondary School Sports Coordinators, and how these behaviours contribute to organisational effectiveness (as operationalised by coaches' job satisfaction, extra effort, and perceived leader effectiveness).

The major aims of the study are:

1. Determine the leadership behaviours of Wellington Secondary School Sports Coordinators (transformational, transactional, or laisse-faire).

2. Explore the relationships between each leadership category and perceived organisational effectiveness.

3. Confirming or not the applicability's of previous studies frameworks and findings on transformational leadership and organisational effectiveness to the context of Wellington Secondary School Sport.

What type of participants are being sought? 
We are seeking 3-5 coordinators who are willing to give up roughly 30 minutes to an hour of their time to engage in a semi-structued interview at their convenience.

\section{What will participants be asked to do?}

Should you agree to take part in this project, you will be asked to participate in a semi-structured interview seeking information on leadership behaviours, and how these relate to perceived organisational effectiveness of your school's sports provision.

The interview will be treated with confidentiality, that is, pseudonyms will be used to ensure that all information gathered will not be traceable back to yourself and your school.

\section{What if participants have any questions?}

If you have any questions about our project, either now or in the future, please feel free to contact either:

Tom Adamson (Student Researcher)

School of Management

Email: adamsotom@vuw.ac.nz

Phone: 0273040973 or, Professor John Davies

School of Management

Email: john.davies@vuw.ac.nz 


\section{Appendix D}

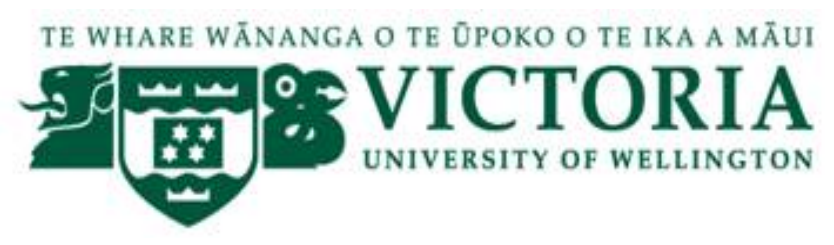

INTERVIEW GUIDE

\section{(1) OPENING QUESTIONS - Participant/School Information}

1. Can you briefly tell me about yourself, background, and involvement as a sports coordinator?

a. Note down age, gender, years in role/experience etc.

2. Similarly, what can you tell me about your school?

a. PROMPTS - Student population and numbers participating in sport; team, individual and coaching roles within sporting provision?

1. How would you summarize the current state of sport in your school?

a. PROMPT - Teams, participants, coaches, manager, funds and facilities; what/who are involved and are they adequate?

2. In terms of funding, do you have a budget?

a. Do you know where this comes from; and do you believe it to be adequate for what you want to do; for quality provision? i.e. Kiwisport funding.

1. Does the school board/council have an explicit policy on sport? If so, can you briefly summarize it?

2. In relation to other departments within the school, where would you say sport sits in terms of importance and emphasis for the school?

3. Is there a formal coordinating group or individual responsible for sport provision?

a. If so, what is the make up of the sport group? (teachers, administrators, coaches etc?... permanent or temporary?...

4. In your role, what relationships do you have? (formal or otherwise) - For instance, do you commonly deal with staff members, coaches, and wider sporting organisations?

a. PROMPTS - Within the school, for whom are you responsible?

b. Whom do you report to?

c. With whom do you frequently interact the most with?

d. What relationships do you consider most important to ensuring continual quality provision? 


\section{(2) LEADERSHIP QUESTIONS}

\section{Personal thoughts on leadership in sports coordinators}

1. Can you tell me a bit about what you think makes a good sports coordinator?

2. Do you have any examples of good coordinators, such as their traits and behaviours or things they've done?

3. Changing the topic slightly, what do you think makes a good leader in the sport coordinator role?

4. What do you think makes a poor leader in the coordinator role? Examples and traits/behavs

5. Turning to yourself, can you say whether you've displayed some of these behaviours or traits?

6. Can you provide me with any recent examples of your leadership as a sports coordinator?

7. Are you conscious of having a leadership style of your own?

8. How and why do you think this behaviour as a sports coordinator is important to the school sport environment?

9. You've commented on the people who have been good or poor leaders; to what extent have they influenced you? Examples..

10. What are some of the other influences on your leadership approach/style?

11. Do you believe you modify your styles/approaches depending on the relationships or situations?

12. How do you think other people view your style of leadership?

\section{Transformational Leadership}

1. Can you provide any examples of these leadership styles within secondary school sport coordinators and staff? - DO ONE AT A TIME

2. If you turn to the handout I have provided you, which behaviours do you see yourself use?

3. Based on these examples, do you think that transformational leadership can be a valuable asset for a sports coordinator within secondary school sport? (PROMPTS overcoming financial/structural/personnel constraints)

a. From your experience, based on the examples you mentioned, is it a common feature of sport coordinator's behaviour? Why or why not?

b. Do you believe there should be leadership training for sports coordinators?

i. Would this be likely to improve the quality of provision?

4. So, of these behaviours, which ones do you believe could be effective/most effective in your role?

5. Out of transactional and laissez-faire styles, which behaviours would be most ineffective and why? Also, what about transformational behaviours?

6. As leadership styles have been shown to effect others in the work unit; how do you, or how can you, get the best performance from your followers (coaches, administrators, teachers, other adjunct staff)?

a. Which of these behaviours (see handout) would you employ for this? 


\section{(3) ORGANISATIONAL OUTCOMES}

1. Subordinate job satisfaction - on a scale of 1-5, how satisfied do you believe your followers are with your leadership?

2. Extra effort

3. On the scale, how effective do you think they would rate your leadership behaviours?

4. Of the three above outcomes of your leadership, how important do you believe them to be in terms of their influence on the effectiveness and quality of sporting provision?

\section{(4) INTERVIEW HANDOUT}

\section{Transformational Leadership}

A leader who influences major changes in attitudes and assumptions of organisational members and build commitment for the organisation's mission and objectives.

1. CHARISMA/IDEALIZED INFLUENCE - influence followers through their developed trust, respect, and loyalty to work beyond their self-interest in order to achieve common goals.

2. INSPIRATIONAL MOTIVATION - articulate a vision of the future and motivate and inspire their followers to commit to such desired goals.

3. INTELLECTUAL STIMULATION - stimulate innovation and creativity through questioning old assumptions, traditions and beliefs.

4. INDIVIDUALIZED CONSIDERATION - leaders pay special attention to each individual follower's needs and act as a coach or mentor.

\section{Transactional Leadership}

A traditional view of leadership whereby the leader outlines the work that must be done by followers, how it will be done, and the rewrads that will be received for successfully completing the objectives.

1. CONTINGENT REWARD - clarifying the work that must be achieved and use rewards in exchange for good performance.

2. ACTIVE MANAGEMENT-BY-EXCEPTION - leaders actively monitoring the work of followers and making sure that standards are met.

3. PASSIVE MANAGEMENT-BY-EXCEPTION - only intervening when problems arise.

\section{Laissez-Faire Leadership}

Does not display any leadership behaviours or traits; rather, they avoid accepting their responsibilities, are absent when needed, fail to follow up requests for assistance, and resist expressing their views on important issues. 


\section{Appendix E}

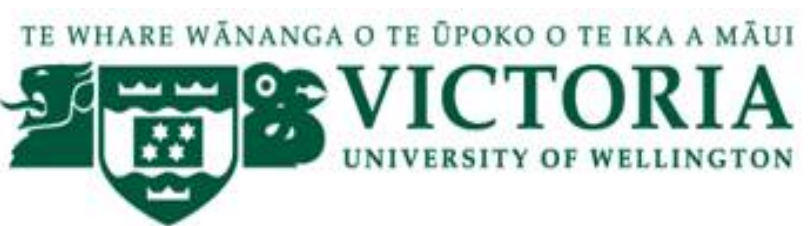

\section{SURVEY QUESTIONNAIRE: MULTIFACTOR LEADERSHIP QUESTIONNAIRE SELF FORM EXAMPLES}

\section{Research project:}

\section{Transformational Leadership and Organisational Effectiveness in Wellington Secondary School Sport Coordinators}

This questionnaire survey will help you describe your sports coordinator's leadership style as you (the coach) perceive it.

Starting with the first statement, judge how frequently each statement fits your role as school sport coordinator.

For items 1 through 18 , circle the number $(0,1,2,3,4)$, that most closely resembles your judgement.

\begin{tabular}{|ccccc}
\hline Not at all & Once in a while & Sometimes & Fairly often & Frequently, \\
& 1 & 2 & 3 & \\
0 & & & \\
\hline
\end{tabular}

\begin{tabular}{|c|c|}
\hline 1. & The sports coordinator $(\mathrm{S} / \mathrm{C})$ avoids getting involved when important issues arise ......................................... 01234 \\
\hline 2. & 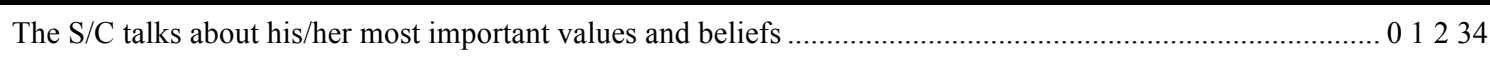 \\
\hline 3. & 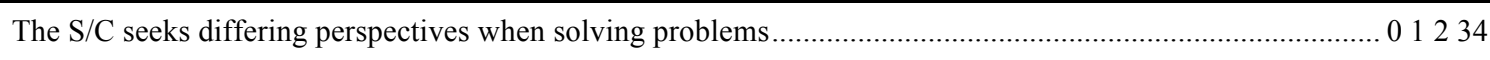 \\
\hline 4. & The $\mathrm{S} / \mathrm{C}$ discusses in specific terms who is responsible for achieving performance targets ......... \\
\hline 5. & 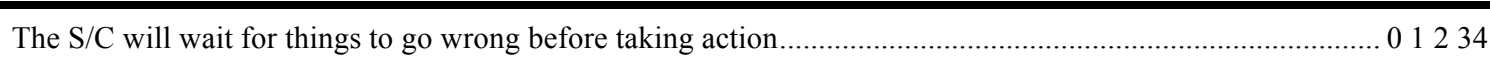 \\
\hline
\end{tabular}

